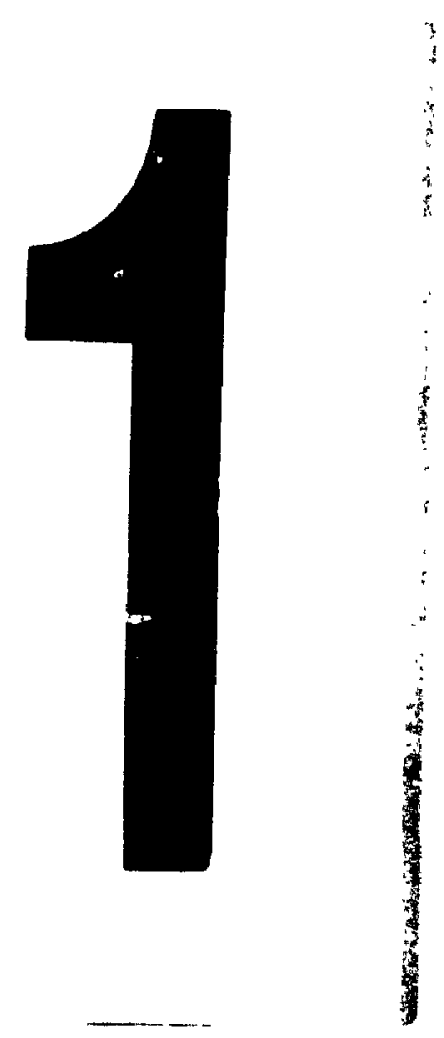

PM-1 3K" $X 4$ " PHOTOCRAPHC MHCROCOPY TAREET MBS 10100 ANSI/1SO H2 ECUNALENT

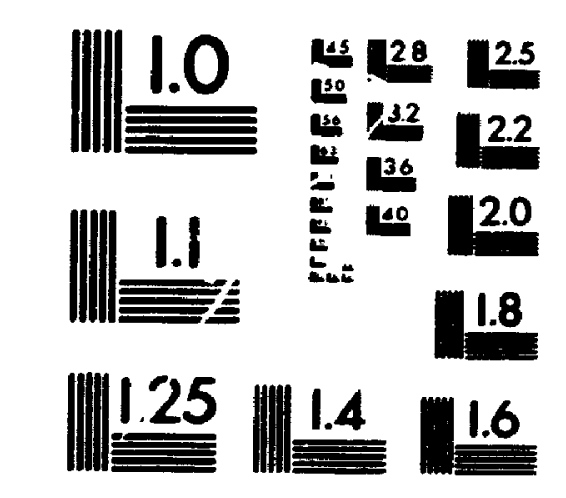

PRECISIONMW RESOUTION TARGETS

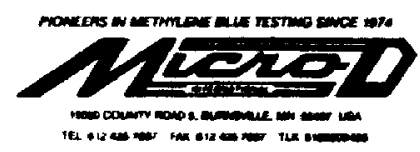


The quality of this microform is heavily dependent upon the quality of the original thesis submitted for microlilming. Every effort has been made to ensure the highest quality of reproduction possible.

If pages are missing, contact the university which granted the degree.

Some pages may have indistinct print especially if the original pages were typed with a poor typewriter ribbon or if the university sent us an inferior photocopy.

Reproduction in full or in part of this microform is governed by the Canadian Copyright ACt, R.S.C. 1970, C. C-30, and subsequent amendments.
La qualité de cette microlorme dípend grandement de la qualité de la thèse scumise au microtilmage. Nous avons tout lait pour assurer une qualiné supérieure de reproduction.

S'il manque des pages, veuillez communiquer avec runiversité qui a contéré le grade.

La qualité d'impression de certaines pages peut laisser à désirer, surtoul si les pages originales ont élé dactylogra. phiées à taide ơun nuban usé ou si runiversité nous a fant parvenir une photocopie de qualité intérieure.

La reproduction, méme partielle. de cette microlorme est soumise a la Loi canadienne sur le droit dauteur. SAC 1970, c. C-30. et ses amendements subsequents. 


\title{
A Cascadeable Pipelined Fast Fourier Transform Switch With Built-In Self-Test
}

\author{
by \\ Clifford Po Shek Yeung, B.Sc.
}

A thesis submitted to the Faculty of Graduate

Studies and Research in partial fulfillment of the requirements for the degree of

Master of Engineering

Ottawa -Carleton Institute for Electrical Engineering

Department of Electronics

Faculty of Engineering

Carleton University

Ottawa, Canada

January, 1992

(C) 1992, Clifford P.S. Yeung 
National Library

of Canada

Canadian Theses Service

Oterwa. Canada

KIA ONA
Brofiotheque nationale du Canada

Service des thitses canadiennes
The author has granted an irrevocable nonexclusive licence allowing the National Lbrey of Canada to reproduce, loan, distribute or sell copies of his/her thests by any meens and in any form or format, making this thesis avelibble to interested persons.

The author retains ownership of the copyright in his/her thesis. Neither the thesis nor substantial extracts from it may be printed or otherwise reproduced without his/her per. mission.
L'auteur a accond'́ une licence intriocable et non exchusive permettent a la Bibiotheque nationale du Canada de reprodulre, preter. distribuer ou vendre des coples de sa these de quelque meniere et sous quelque forme que ce soit pour mettre des exemplaires de cette thése à la disposition des personnes intéressées.

L'auteur conserve la propriété du droit d'auteur qui protége sa these. Ni la these ni des extraits substantiels de celle-ci ne dolvent etre imprimés ou autrement reproduits sans son autorisation. 
Thesie containe black and white and/or coloured graphe/tebles which when microfilmed wey loee their elgnificerice. The herdcopy of the theese is aveileble upon requeat from Carleton Univeraity Library. 
The undersigned recommend to the Faculty of Graduate Studies and Research acceptance of this thesis

\title{
"A CASCADEABLE PIPELINED FAST FOURIER TRANSFORM SWITCH WITH BUILT-IN SELF TEST"
}

\author{
submitted by Clifford Po Shek Yeung, B.Sc. \\ in partial fulfilment of the requirements for \\ the degree of Master of Engineering
}

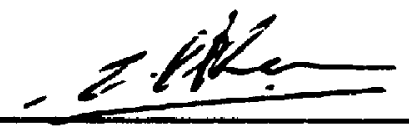

Prof. C.H. Chan

Thesis Supervisor

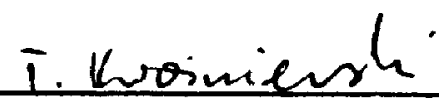

Prof. T. Kwasniewski

Thesis Supervisor

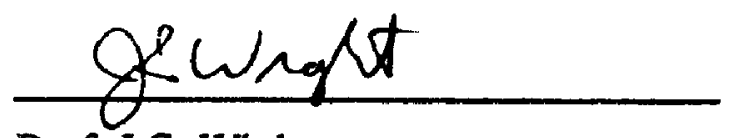

Prof. J.S. Wight

Chairman, Department of Electronics

Carleton University

January, 1992 


\section{Abstract}

The single pipelined fast Fourier transform (FFT) is a one dimensional systolic array composed of two building blocks, namely an FFT Switch and a Complex Butterfly. By partitioning the computation requirements among a number of processing elements, the pipelined architecture offers a speed improvement over the traditional Von-Neumann architecture. A design for the radix-2 pipelined FFT Switch is described in this thesis. The generic design of the proposed architecture enables the FFT Switch to support a number of different transform sizes for a radix-2 FFT processor of up to 8192 points. Additional circuitries are incorporated into the FFT Switch in order to facilitate off-line testing. The Built-in self-test (BIST) is fast becoming an essential technique for verification of complex integrated circuits. The pipelined FFT processor is part of the multi-carrier demodulator intended for on-board digital signal processing for satellite communications. 


\section{Acknowledgements}

The author would like to thank his supervisors, Prof. C.H. Chan and Prof. T. Ku'asniewski for their guidance and support. The author also wishes to thank Dr. V. Szwarc and Mr. L. Desormeaux of Communication Research Centre for their valuable discussions and helpful advice.

The author is very grateful to the staff and graduate students of the Department of Electronics for providing such fertile environment to pursuit academic achievements. The author would also like to express his gratitude towards Mr. C. Foo and Mr. R. Shum for their little help here and there.

The author would like to acknowledge the financial suppon of Communications Canada, Carleton University and Telecommunications Research Institute of Ontario (TRIO). The collaboration of the Alberta Microelectronics Centre is also gratefully acknowledged.

The author would like to thank his parents for their understanding and without their support, this work would not be possible. Finally, the author dedicates this thesis to his younger brother who passed away so tragically at the tender age of six after a brave battle with Leukaemia. You are always in my heart. 


\section{Table Of Contents}

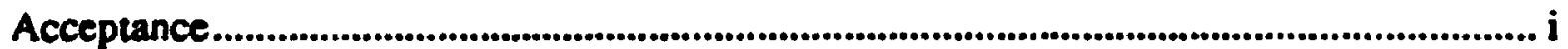

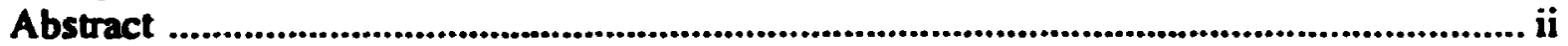

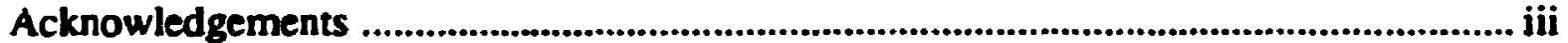

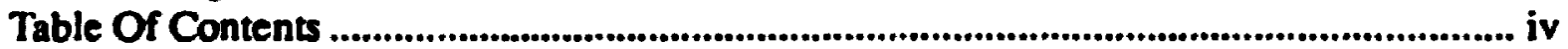

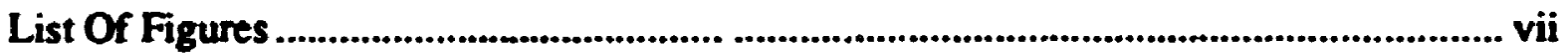

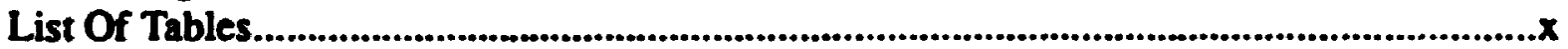

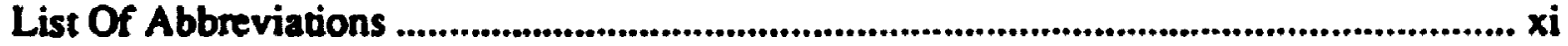

\section{Chapter 1 Introduction}

1.1 Thesis Motivation and Objectives .............................................. 1

1.2 Thesis Contributions ................................................................ 5

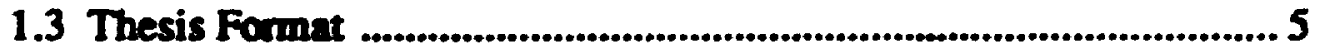

\section{Chapter 2 Fast Fourier Transform Implementations and Fourier Transform Alternatives}

2.1 Introduction

2.2 The Discrete Fourier Transform and Fast Fourier Transform Algorithms

2.3 FFT Implementations Overview ............................................. 11

2.3.1 Introduction ............................................................ 11

2.3.2 General Purpose Digital Systems ............................ 12

2.3.3 General-purpose Digital Signal Processor .................... 13

2.3.4 Dedicated Processors for DFT and FFT ...................... 15

2.3.4.1 Introduction ............................................................ 15

2.3.4.2 Pipelined and Parallel Pipelined FFT Architecture 15

2.3.4.3 Single Chip Approach ........................................... 20

2.3.4.4 Multichip Approach ...................................... 21

2.4 Summary ...................................................................... 23 
Chapter 3 Architectural developments for the Pipelined Fast Fourier Transform Chip Set

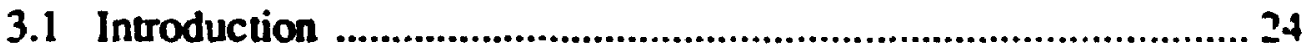

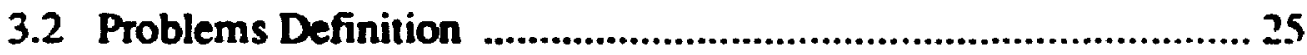

3.3 Architectural Considerations for the FFT Switch ...................... 26

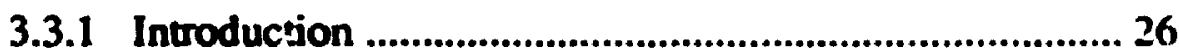

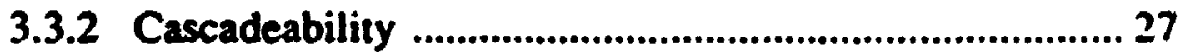

3.3.3 Switch Control ................................................................ 30

3.3.4 Testability Issues ........................................................ 32

3.4 Architectural Considerations for the Complex Butterfly ........... 34

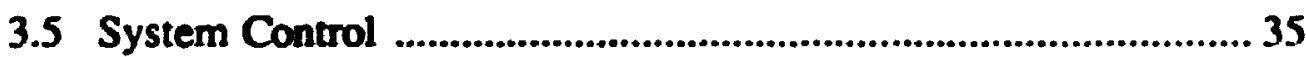

3.6 Functional Simulation of the Pipelined FFT ...............................36

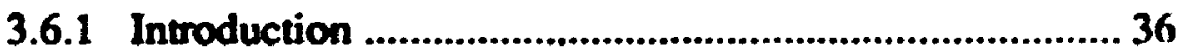

3.6.2 Models for the FFT Switch and the Complex Butterfly 37

3.6.3 Simulation Results ......................................................... 39

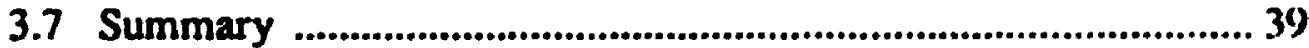

\section{Chapter 4 Circuit Description of the FFT Switch}

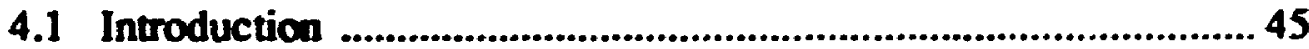

4.2 Computer Aided Design Tools ................................................... 46

4.2.1 Introduction ..................................................................46

4.2.2 Schematic Capture Tools ............................................ 46

4.2.3 Simulation Tools .......................................................... 47

4.3 Design Implementation .............................................................48 48

4.3.1 Design of the Delay Circuit and Commutator blocks ... 48

4.3.2 Control Unit Design ................................................... 53

4.3.2.1 Introduction .......................................................... 53

4.3.2.2 The Clock Cycles Generator .................................53

4.3.2.3 The Sync Pulse Logic (SPL) ................................55 55

4.3.2.4 The Cascadeability Control ................................ 56

4.3.2.5 Information Latch ..........................................5 57

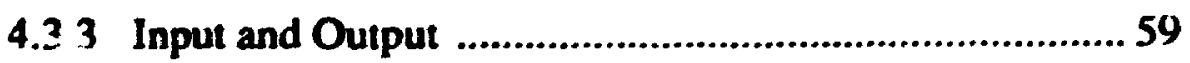

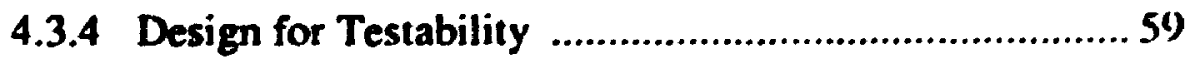


4.3.4.1 Built-in Self-test ..................................................59

4.3.4.2 Interconnectivity Test .......................................... 64

4.4 Summary ............................................................................................65

\section{Chapter 5 Simulation and Test results}

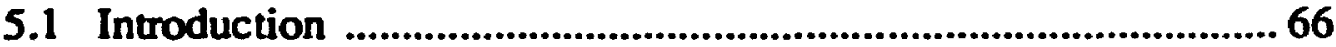

5.2 Simulations of the FFT Switch .................................................67

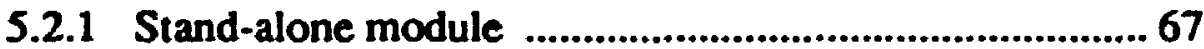

5.2.1.1 Introduction ..........................................................67 67

5.2.1.2 Functional Test .................................................67 67

5.2.1.3 Non-functional Simulations ............................. 77

5.2.2 Cascaded Structure Simulations .................................. 82

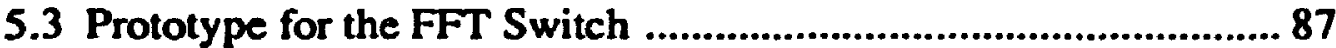

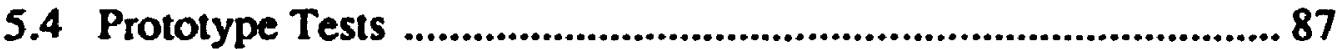

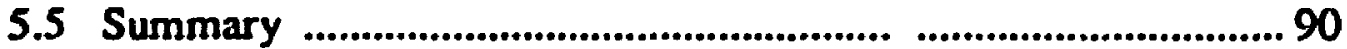

Chapter 6 Conclusions

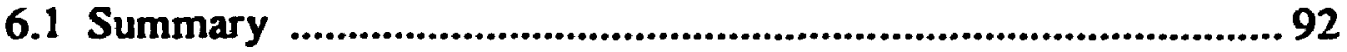

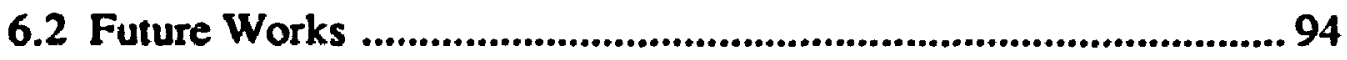

Reference.

Appendices.

Appendix A

Appendix B

Appendix C

Appendix D

Appendix E 


\section{List Of Figures}

Figure 1.1 A Multi-channel Sutellite Communication System .................................... 3

Figure 1.2 Polyphase Filten cascade with FFT Processor ........................................... 4

Figure 2.1 A parly decomposed 16 point signal flow diagram ................................ 10

Figure 2.2 A fully decomposed flow diagram of a 16-point FFT. .......................... 12

Figure 2.3 A Generic Pipelined Architecture. .................................................................. 16

Figure 2.4 A Block Diagram of a Single pipelined Fast Fourier Transform. ........... 17

Figure 2.5 A 32-point radix-2 pipelined fast Fourier transform .............................. 18

Figure 2.6 A 32-point Radix-2 parallel pipelined fast Fourier transform ................. 19

Figure 2.7 A timing diagram of the single pipelined FFT. ......................................... 20

Figure 2.8 A timing diagram of the parallel pipelined FFT. ....................................... 21

Figure 3.1 A block diagram of the FFT Switch ........................................................... 26

Figure 3.2 Five modes of operations ...................................................................... 28

Figure 3.3 A block diagram of a 128 point pipelined FFT processor ....................... 29

Figure 3.4 Four cascaded Switches .................................................................................... 30

Figure 3.5 A diagram to illustrate the operation of the Sync pulse. .......................... 31

Figure 3.6 A built-in self-tent arrangement. ............................................................. 33

Figure 3.7 A Block Diagram of a Complex Butterfly ...................................................... 34

Figure 3.8 A model for FFT Switch. ............................................................................. 37

Figure 3.9 A model for the Complex Butterfly. .................................................................. 38

Figure 3.10 Operations of the commutator ......................................................................... 38

Figure 3.11 Model code for the Delay Element ........................................................... 39

Figure 3.12 Model code for the Commutator .............................................................. 40

Figure 3.13 Model code for the Complex Bunerfly .................................................... 41

Figure 3.14 Model code for the Angle Block ........................................................... 42

Figure 3.15 The simulated Splice output with a $2 \mathrm{KHz}$ input source ........................ 43

Figure 3.16 The simulated Splice output with a $10 \mathrm{KHz}$ input source ..................... 43

Figure 4.1 A block diagram of a FFT Switch ............................................................ 48 
Figure 4.2 Data channels of Left Switch mode 49

Figure 4.3 A two bits delay line structure 50

Figure 4.4 A Commutator with four sets of multiplexers ......................................... 52

Figure 4.5 A Clock Prescaler .........................................................................................54 54

Figure 4.6 Delay elements of a 32 point pipelined FFT processor .......................... 55

Figure 4.7 Operation of the 32 point pipelined FFT ......................................................... 58

Figure 4.8 A Linear Feedback Shift Register ........................................................... 62

Figure 4.9 A Multiple Inpen Preudorandom Compacter ...........................................6 63

Figure 4.10 An input buffer with a NAND gate for a parametric NAND tree. ........65 65

Figure 5.1 A 32 point pipelined FFT processor ......................................................68 68

Figure 5.2 Simulation result of Switch A of Example 1 ...........................................6 69

Figure 5.3 Simulation result of Switch D of Example 1 ............................................. 71

Figure 5.4 A two Switches cascaded stnucture ............................................................. 72

Figure 5.5 Simulation result of End switch mode in Example 2 ................................. 73

Figure 5.6 Simulation result of Output switch mode in Example 2 ........................... 74

Figure 5.7 A four Swivehes cancaded structure ............................................................. 75

Figure 5.8 Simulation result of End switch mode in Example 3 ............................... 76

Figure 5.9 Simulation result of Left switch mode in Example 4 ............................... 78

Figure 5.10 Simulation result of Right switch mode in Example 4 ........................... 79

Figure 5.11 Simulation result of Output switch mode in Example 4 .........................8 80

Figure 5.12 Simulated response of the GO_NOGO output ......................................... 81

Figure 5.13 Three two modules combinations include a Switch ................................8 83

Figure 5.14 Simulation result of Example 6 ..................................................................... 85

Figure 5.15 Simulation result of Example 7 .............................................................. 86

Figure 5.16 A die photo of the FFT Switch ...........................................................8 88

Figure A.1 The simulation result of Switch B in Example 2...........................................101

Figure A.2 The simulation result of Switch $C$ in Example 2 ...........................................102

Figure B.1 The simulation result of the Left Switch mode in Example 3 ......................104

Figure B.2 The simulation result of the Right Switch mode in Example 3 .....................105

Figure B.3 The simulaticn result of the Output Switch mode in Example 3 ...................106 
ix

Figure C.1 Operation of a parametric NAND tree.

.109

List of Figure 


\section{List of Tables}

Table 5.1 A table of all possibilities for "Switch-Switch" combination ................. 84

Table 5.2 "Swit":h-Butterfly" and "Butterfly-Switch" combinations .................... 87

Table 5.3 Prototype results at self-test mode for 5.0 V power supply .................... 89

Table 5.4 Prototype results at self-test mode for 3.3V power supply ..................... 90 


\section{List of Abbreviations}

$\begin{array}{ll}\text { ADC } & \text { Analog to Digital Converter } \\ \text { ASIC } & \text { Application Specific Integrated Circuit } \\ \text { BIST } & \text { Built-in Self-test } \\ \text { CAD } & \text { Computer Aided Design } \\ \text { CFT } & \text { Continuous Fouier Transform } \\ \text { CMOS } & \text { Complementary Metal Oxide Semiconductor } \\ \text { CRC } & \text { Communication Research Centre } \\ \text { CS } & \text { Chip Select } \\ \text { DFT } & \text { Discrete Fourier Transform } \\ \text { DIT } & \text { Decimation In Time } \\ \text { DSP } & \text { Digital Signal Processor } \\ \text { FDMA } & \text { Frequency Domain Multiplexing Access } \\ \text { FFT } & \text { Fast Fourier Transform } \\ \text { GPDS } & \text { General Purpose Digital System } \\ \text { HCMOS } & \text { High Speed CMOS (LSI Logic) } \\ \text { IC } & \text { Integrated Circuit } \\ \text { LFSR } & \text { Linear Feedback Shift Register } \\ \text { MCD } & \text { Multi-carrier Demodulator } \\ \text { MCM } & \text { Multichip Module } \\ \text { MIPC } & \text { Multiple Input Pseudorandom Compacter } \\ \text { MSPS } & \text { Million Samples per Second } \\ \text { RAM } & \text { Random Access Memory } \\ \text { ROM } & \text { Read Only Memory } \\ \text { SPL } & \text { Sync Pulse Logic } \\ \text { TDM } & \text { Time Domain Multiplex } \\ \text { VII } & \text { Logic low input } \\ \text { VIH } & \text { Logic r.igh input } \\ \text { VLSI } & \text { Very L.rge Scale Integrated circuits } \\ & \end{array}$




\section{Chapter One}

\section{Introduction}

\subsection{Thesis Motivation and Objectives}

Since the development of the Cooley-Tukey fast Fourier transform (FFT) algorithms [1] over two decades ago, there has been a great deal of advancement in the area of hardware FFT implementation. However, it is only recently that VLSI technology has enabled the realization and exploitation of this algorithm in such realtime applications as radar detection, image processing or frequency-division multiple access (FDMA) to time-division multiple access (TDMA) signal conversion. Furthermore, current advances in the field of very high speed Analogue to Digital Converters (ADC) enhance data acquisition technique and allow the integration of advanced FFT processors with throughputs of the order of tens of millions of samples per second as part of the final system. 
Due to the regular structure of this algorithm and its ability to compute in-place, it is particularly popular for FFT implementation and a number of different approaches have been used. The FFT algorithm can be computed by programming a microprocessor or a general purpose digital signal processor. However, for critical applications where very high throughputs are essential, general purpose digital signal processors and even dedicated FFT processors may not be adequate. One way to increase the throughput of an FFT processor is to partition the computational requirements among a number of processing elements. The pipelined FFT does this by mapping the FFT algorithm onto a one dimensional systolic array. The processor can be implemented by means of two basic building blocks, namely an FFT Switch (Switch) and a Complex Butterfly. The basic function of the FFT Switch is to provide the necessary clock delays and to reorder and rout the data between the Complex Butterflies, which are responsible for all numerical computations.

As the demand for satellite communications increases rapidly, especially in the commercial sectors, the need for low space-segment tariffs and simple and cheap earthterminals is growing. One promising technique which could meet these objectives would use FDMA as the up-link to the satellite and TDMA for the down-link to ground stations, providing that the necessary processing is done on-board the satellite[2]. Figure 1.1 shows the basic concept of the system. This technique demonstrates that multi-channels communication can be accomplished by employing low cost earthterminals with the expense of extra processing hardware on-board the satellite. The specific hardware in question is the multi-carrier demodulator (MCD), which permits the direct on-board interfacing of FDMA and TDMA communication links by digital signal processing techniques. One part of the MCD is a demultiplexer which can be 


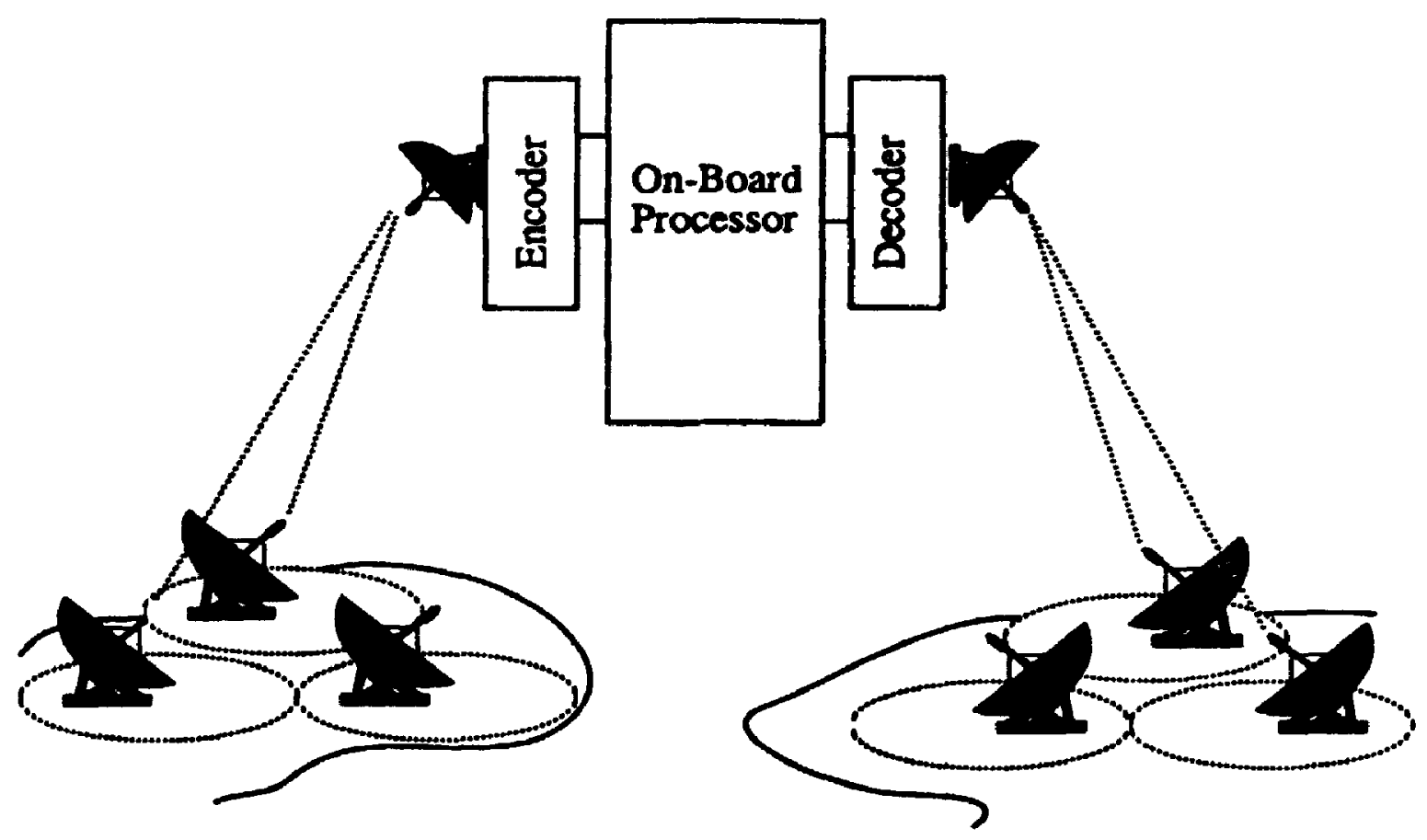

Figure 1.1 A Multi-channel Satellite Communication System

implemented by means of a digital polyphase filter bank cascaded with an FFT processor. Figure 1.2 shows the arrangement of the demultiplexer. The initial impetus for this work has been the requirement to develop a 25 Msample/sec (MSPS) per channel 32-point radix-2 FFT processor for the demultiplexer. Although other techniques [3] can be used to implement the demultiplexer, the FFT design is particularly efficient for the situations where a large number of communication channels are present.

The FFT chip set is designed to support the implementation of a wide range of pipelined radix-2 FFT processors. In order to accomplish this, both the FFT Switch and Complex Butterfly must function as generic building blocks capable of operating at different locations of the pipelined processor. The implementation of the FFT Switch is 


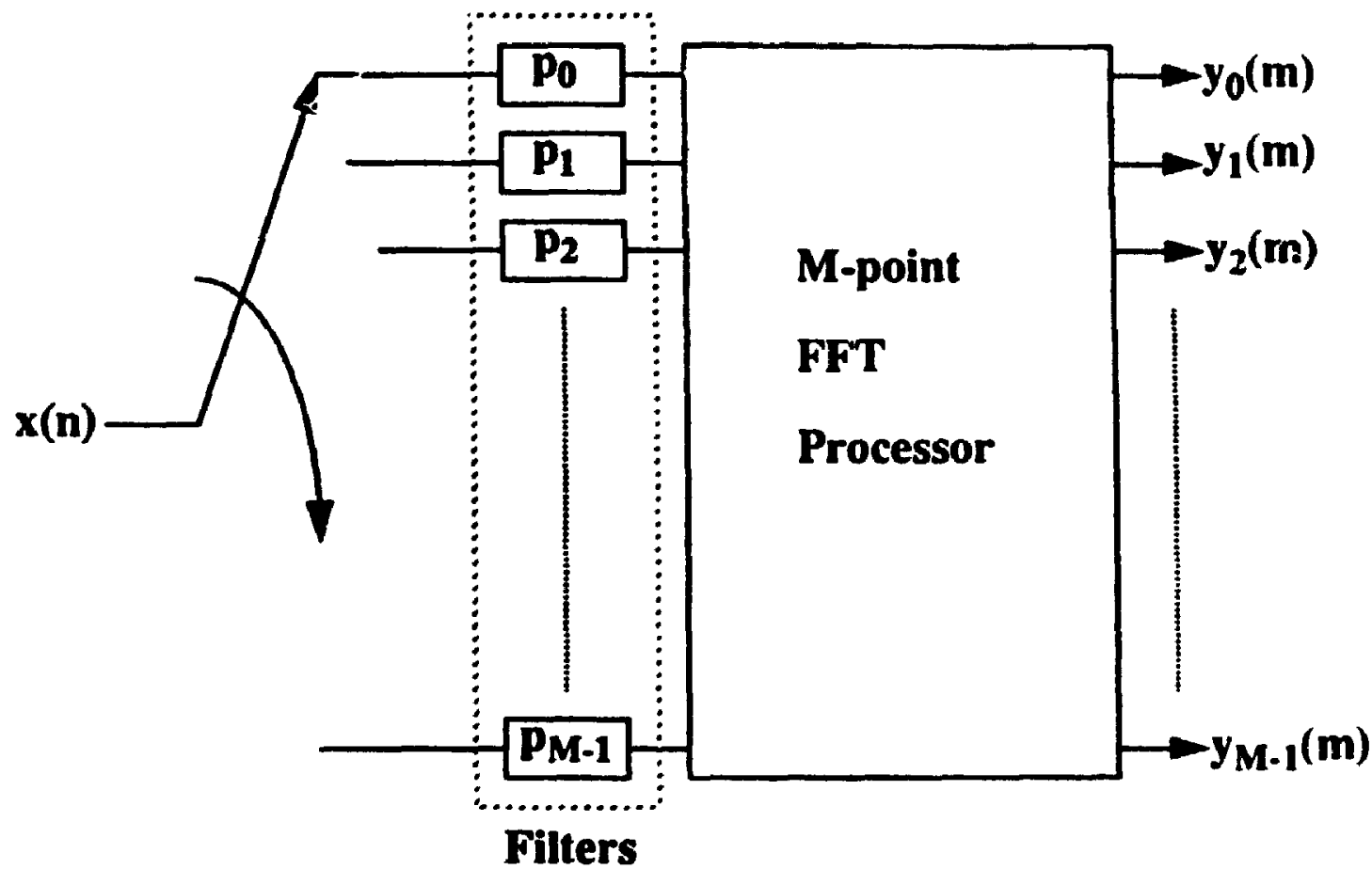

Figure 1.2 Polyphase Filters cascade with FFT Processor

the focus of this thesis and a description of the Complex Butterfly can be found in the literature [22].

The objective of this thesis is to develop a generic FFT Switch capable of implementing a very high speed radix-2 FFT. Furthermore, the FFT Switch must be cascadeable in order to provide the necessary number of clock delays for higher transform sizes which a single Switch cannot accommodate due to technological limitations. A portion of the Switch circuitry must be dedicated to facilitate testing. The self-test approach is particularly attractive in the testing of integrated circuits with a large number of pin out. 


\subsection{Thesis Contributions}

By utilizing the pipelined architecture, a chip set is designed for high throughput fast Fourier transform implementation and a FFT Switch architecture is proposed in the thesis. The proposed generic architecture of a FFT Switch demonstrated that by exploring the regular structure of the pipelined FFT algorithm and the in-place combination, it is possible to implement a spectrum of transform sizes by using only two integrated circuits in the chip set. The design of the Switch is cascadeable and it shows that, without the need for further external memory elements, it is possible to provide the necessary number of delay for the pipelined architecture. Moreover, the flexibility of the Switch design enables to support the implementation of the forward and inversed, decimation-in-time and decimation-in-frequency radix-2 FFT algorithms.

\subsection{Thesis Format}

In Chapter 2, a broad survey on the fast Fourier transform implementations and alternatives is presented. Among the available Fourier transform implementations, a pipelined FFT processor provides the highest performance in terms of data throughput. A more thorough investigation into the pipelined FFT chip set and in particular the FFT Switch is provided in Chapter 3. In Chapter 4, the FFT Switch is partitioned into separate functional blocks and the circuitry in each block will be described in detail. Simulation and test results are presented in Chapter 5 in order to verify the functionality of the Switch. Chapter 6 provides a summary and suggestions for future work. 


\section{Chapter Two}

\section{Fast Fourier Transform Implementations and Fourier Transform Alternatives}

\subsection{Introduction}

For many years, the Fourier transform has been a universal technique which enables one to examine a particular relationship from an entirely different poin: of view. It identifies the frequency components which make up a continuous waveform. Unfortunately, as a continuous and infinite process, the Fourier transform is not amenable to the machine discrete time computation adopted by all digital systems. However, a special type of Fourier transform suitable for digital hardware and software implementations has been proposed, and is called the discrete Fourier transform (DFT). The DFT is the sampled representation of the continuous Fourier transform (CFT), which operates over a finite time interval. For a sufficiently small sampling interval and large number of sampled points it asymptotically approximates CFT. A class of efficient 
algorithms to compute the DFT is known as the fast Fourier transform (FFT). One such algorithm was developed by Cooley and Tukey [1]. This now commonly used FFT algorithm is one of the most important operations encountered in signal processing applications today.

The FFT, with it: substantial savings in computer time, can be used in place $u$. the continuous Fourier transform only to the extent that the DFT could be previously. Many applications, such as digital convolution, spectrum analysis, audio and video signal processing and so on, are able to utilize the FFT as an efficient technique to perform computations. Furthermore, through VLSI technology, many FFT processes are able to operate with the very high clock frequency necessary for real-time applications.

In the next section, the DFT and FFT algorithms are examined. This is followed by an implementation overview of the FFT which includes an outline on the pipelined and parallel pipelined architectures.

\subsection{The Discrete Fourier Transform and Fast Fourier Transform Algorithms}

The Fourier transform function for continuous signals can be written in the form of Equation (2.1)

$$
X(f)=\int_{-\infty}^{\infty} x(t) e^{-j 2 \pi f t} d t
$$


for $-\infty<f<\infty$ and $-\infty<t<\infty$. The $\left.X()^{\prime}\right)$ represents the frequency-domain function and $x(t)$ is the time-domain function.

Although a more complete examination of fast Fourier transform should begin with a detailed look at the continuous Fourier transform, since it is not suitable for digital computation the emphasis will be placed on the DFT.

As mentioned in the previous section, the DFT can be viewed as the sampled representation of CFT and sampled waveform can be expressed in Equation (2.2).

$$
x(n)=x(t) \sum_{k=-\infty}^{\infty} \delta(t-k T)
$$

Sampling is accomplished by multiplying the continuous time-domain signal $x(t)$ with a time-domain periodic impulse train, $\sum_{k=-}^{-} \delta(t-k T)$. A time difference $T$, between periodic sampling impulses, is called the sampling interval or sampling frequency for its reciprocal. In order to avoid aliasing and loss of information, the continuous waveform must be sampled at a frequency of at least twice the largest frequency component of the usually bandlimited continuous signal $x(t)$. Once the sampled time-domain waveform is obtained, further computation to transform the data to frequency-domain is completed by using a discrete-time process. The analogous discrete Fourier function can be expressed in the form of Equation (2.3) 


$$
X(k)=\sum_{n=0}^{N-1} x(n) W_{N}^{k n}
$$

where $W_{N}=e^{-j((2 \pi) / N)} k=0,1,2, \ldots, N-1$ and $N$ is the number of transform points. The $x(n)$ is a complex data sample in the time-domain and $X(k)$ is the complex image of the data with frequency-domain.

Since continuous Fourier transform is an infinite process where an infinite number of samples will be introduced, it is necessary to truncate the sampled function $x(n)$ so that only a finite number of points, say $N$, are considered. Examination of equation (2.3) reveals that $N^{2}$ complex multiplications and $N(N-1)$ complex additions are necessary to perform direct computation [4]. As each complex multiplication represents four real multiplications and two real additions, the algorithm becomes extremely computationally expensive as $\mathbf{N}$ increases. In addition to the amount of computation being required, provisions have to be made for storing and accessing the $\mathbf{N}$ complex input sequence $x(n)$ and the values of complex coefficients. Fortunately, an efficient class of algorithms has been developed to compute the DFT, collectively known as the fast Fourier transform (FFT). Such algorithms exploit the symmetry property and periodicity properties of $W_{N}^{k n}$ as shown in Equations (2.4) and (2.5) respectively [5].

$$
\begin{aligned}
& W_{N}^{k(N-n)}=W_{N}^{k n}=\left(W_{N}^{k n}\right)^{*} \\
& W_{N}^{k n}=W_{N}^{k(n+N)}=W_{N}^{(k+N) n}
\end{aligned}
$$


where ()$^{*}$ denotes the complex conjugate of () . Further savings can be achieved by using the fundamental principle of decomposing the computation of the discrete Fourier transform of a sequence of length $\mathbf{N}$ into successively smaller discrete Fourier transforms. For the special case where $N$ is an integer power of 2 (i.e. $N=2^{\gamma}$, where $\gamma$ is an integer), it is possible to compute the $X(k)$ by separating $X(n)$ into a number of $(N / 2)$ point sequences. For example, a partly decomposed flow graph of a 16-point FFT algorithm is as shown in Figure 2.1. The process continues until the sequence is fully decomposed. Since the number of complex multiplications for an N-point DFT reduces to

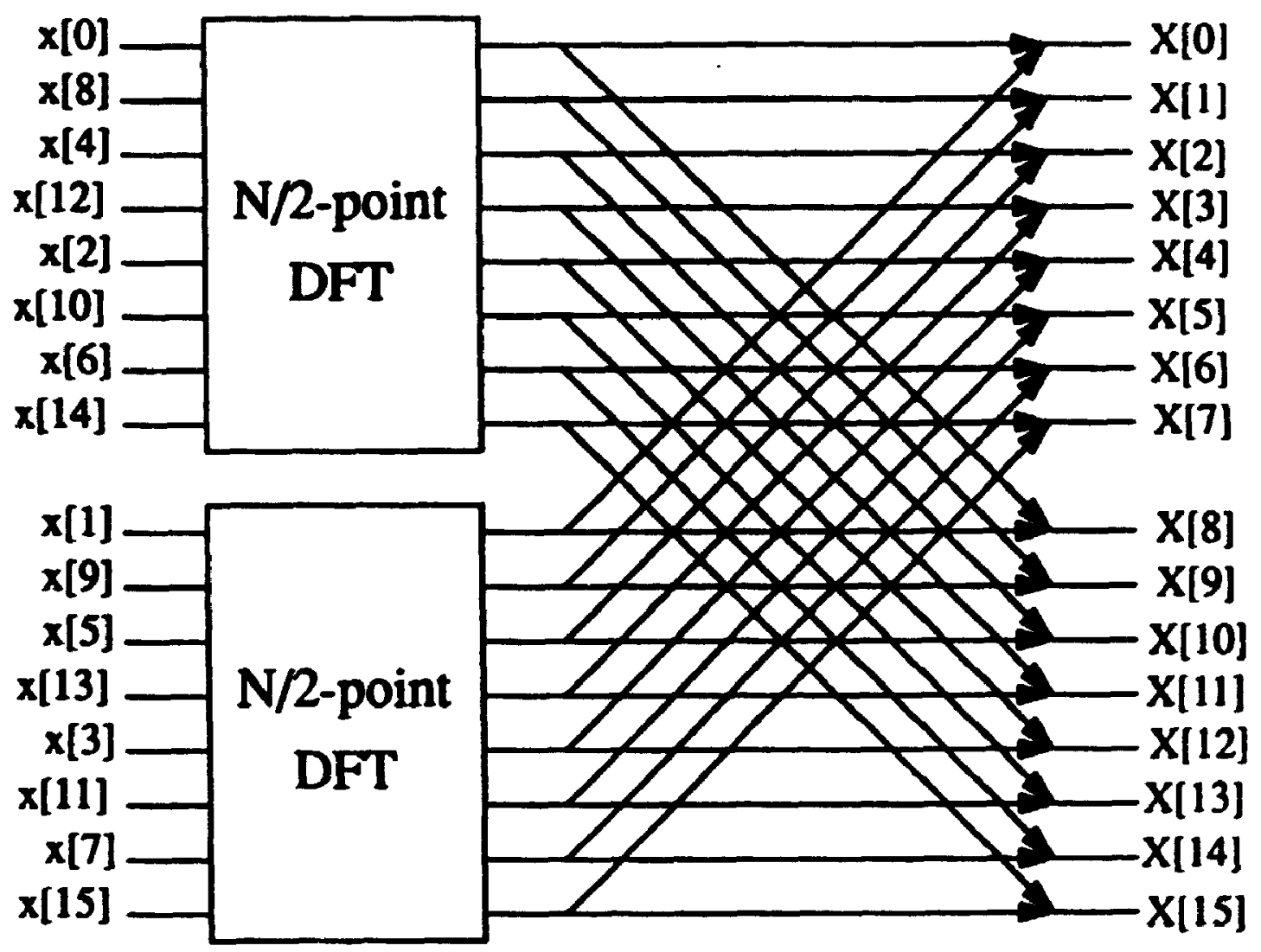

Figure 2.1 A partly decomposed 16 poim signal flow diagram 
$(\mathrm{N} / 2)^{2}$ for a (N/2)-point DFT, the total multiplication requirement of the system for one decomposed step will be $\mathrm{N}+2(\mathrm{~N} / 2)^{2}$. As the (N/2)-point DFT decomposes to two (N/ 4)-point DFT and to four (N/8)-point DFT and so on, it reduces the overall number of complex multiplications from $\mathrm{N}^{2}$ (no decomposition) to $\mathrm{Nlog}_{2} \mathrm{~N}$ (fully decomposed). It is clear that even for a moderately sized $\mathbf{N}$, a considerable reduction in computational operations can be achieved. Further details can be found in the literature [5][6].

A fully decomposed 16-point radix-2 decimation-in-sime (DIT) FFT signal flow diagram is shown in Figure 2.2. Two interesting features of this algorithm are shown in the diagram. First of all, it is possible to perform the in-place computation using this algorithm. An in-place computation requires the least storage capacity during operation and hence it is considered very suitable for the pipeline implementation. Secondly, in order for the computation to be done in-place, it is necessary to either pre-format the input sequence into bit-reversed order before it is accessed or post-format the bitreversed output for a normally ordered input sequence. Although it is possible to arrange the signal flow diagram such that both input and output sequences are normally ordered, the in-place property of the algorithm will be lost [6].

\subsection{FFT Implementations Overview}

\subsubsection{Introduction}

In the previous section, a brief examination of the FFT algorithm and its computational advantage over the DFr algorithm were presented. Substantial computational saving is the primary reason that has led to the acceleration of development in the field of DFT implementation using FFT algorithms. In this chapter, 


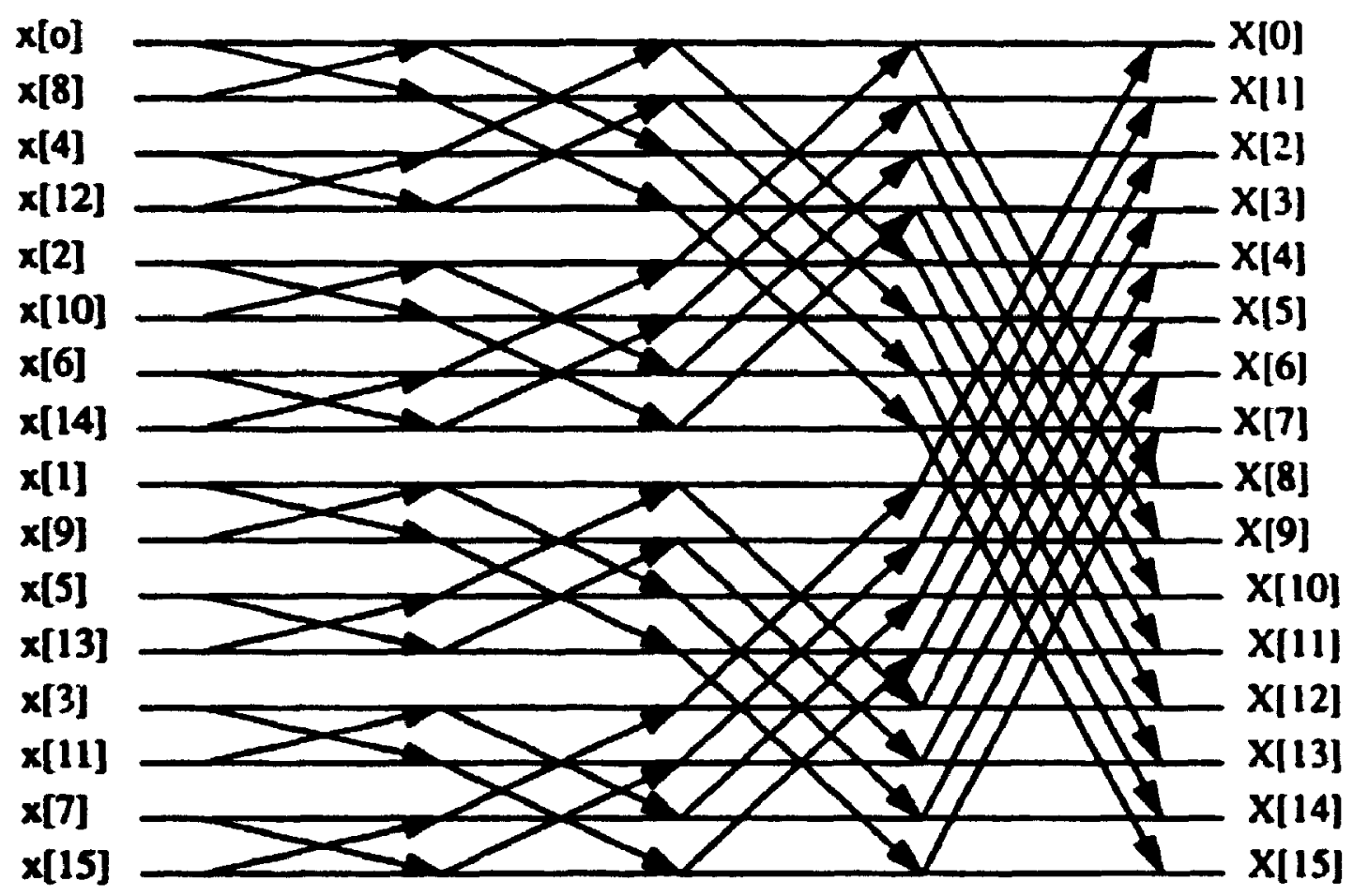

Figure 2.2 A fully decomposed flow diagram of a 16-point FFT.

an overview of the FFT implementations will be provided in the next few sections starting with general-purpose digital systems. This is followed by descriptions of digital signal processors and dedicated FFT hardware.

\subsubsection{General Purpose Digital Systems}

In the early days of FFT use, most implementations were automatically processed on general-purpoce digital systems (GPDS) [7][8][9] where code sequences were written in either assembly or high-level languages to achieve a high degree of flexibility. Furthermore, researchers were able to experiment with various forms of FFT algorithms used in many different applications. Apart from the high degree of 
flexibility, additional techniques have been introduced to upgrade system performance. Most noticeable are the implementations of FFT application specific microcode [7] and the "number crunching" array processors [10]. For the former technique, the FFT algorithm can be partly or completely integrated into microcode at the expense of additional program memory and an extensive amount of programming effort to accomplish the transformation. Although FFT is an efficient algorithm to compute DFT, nonetheless it remains an expensive computational process. Since array processors are capable of high speed mathematical executions, they will significantly improve the overall system performance. In order to operate an array processor effectively, one must obtain parallelism in a particular algorithm and minimize data organizational overheads. Routing between individual processors can be considered as an operation and incurs additional overhead in term of the arithmetic complexity of the algorithm. Moreover, since the cost of such processors is usually high, unless they are used intensively, this may not be a feasible option to take.

Since the execution speed of most general-purpose digital systems is relatively slow compared to that of the dedicated FFT hardware, this implementation will be limited to lower bandwidth applications. Furthermore, the overall system cost and size are ultimately unsuitable both for wide-spread use and for our application in particular.

\subsubsection{General-purpose Digital Signal Processor}

Advances in the development of programmable Digital Signal Processors (DSP) since their commercial introduction in the early 1980 s provide an attractive altemative for computing FFT algorithms. DSPs can support a wide variety of FFT algorithms and a number of FFT implementations have been reported in the literature [11][12]. Similar 
to the general-purpose digital system. DSPs are generally programmed in assembly languages. However, unlike the GPDS, the architecture of the DSP is optimized for signal processing applications where specific numerical operations are required. Researchers have been introducing more and more efficient hardware implementations to fulfil the demands of high FFT executic speed and digital signal processing applications in general. Implementations such as the hardware multiplier, barrel shifier, auxiliary registers and floating point capability enhance the overall performance of the DSPs. The intemal random access memory (RAM) structure of the DSPs is able to store a number of twiddle factors necessary to execute FFT algorithms.

In the past, many signal processing algorithms were carefully structured in order to minimize the number of multiply operations at the expense of more addition and memory operations. Such compromise is inevitable for GPDS due to the fact that multiplications require more computational procedures than both addition and memory operations. As for DSPs, multiplications require the same amount of computation as do the other two operations. Thus some optimized algorithms may not be the most favourable routines to implement on a DSP. However, since FFT algorithms minimize all types of operations, they remain popular as a DSP implementation.

Since the cost of DSPs has been steadily decreasing, they have been introduced into many commercial products. Many existing DSP based digital signal processing systems can be arranged in parallel such that it is possible to achieve greater processing power at the expense of higher system cost. However, VLSI will always be faster than a general purpose DSP. For extremely high speed FFT operations, VLSI processors dedicated to compute DFT and FFT are the only option. 


\subsubsection{Dedicated Processors for DFT and FFT}

\subsubsection{Introduction}

When the execution speeds of previously described techniques are found to be either too slow or $\infty$ costly for the particular application in mind, dedicated VLSI processors optimized for DFT and FFT operations should be used. A number of processor implementations have been reported in the literature. These can be roughly classified into two groups due to their differences in hardware architecture approach, namely single chip processors and multichip processing units. When compared, multichip configuration has the advantage of maximum speed and flexibility over single chip implementation. However, single chip implementation can achieve reasonable performance with lower implementation cost and design effort. Most processors adopt a pipelined architecture approach [13][14] to accomplish the very high performance requirements and maximize the hardware utilization for a given data throughput.

In the next section, the architectures of the pipelined and parallel pipelined FFTs are presented. Selected design features and considerations are highlighted. This is followed by a discussion of single chip and multichip approaches. The multichip approach is adopted in this project.

\subsubsection{Pipelined and Parallel Pipelined FFT Architecture}

Many digital signal processing applications demand an extremely high throughput rate. The pipelined and parallel pipelined FFT processors discussed here may be able to achieve such performance requirements. The one dimensional systolic array (pipelined) architectures generate a great deal of interest since they offer a considerable speed improvement over the traditional Von-Neumann architecture. 
Figure 2.3 shows a generic pipelined architecture and the pipeline is partitioned into $\mathrm{N}$ number of modules.

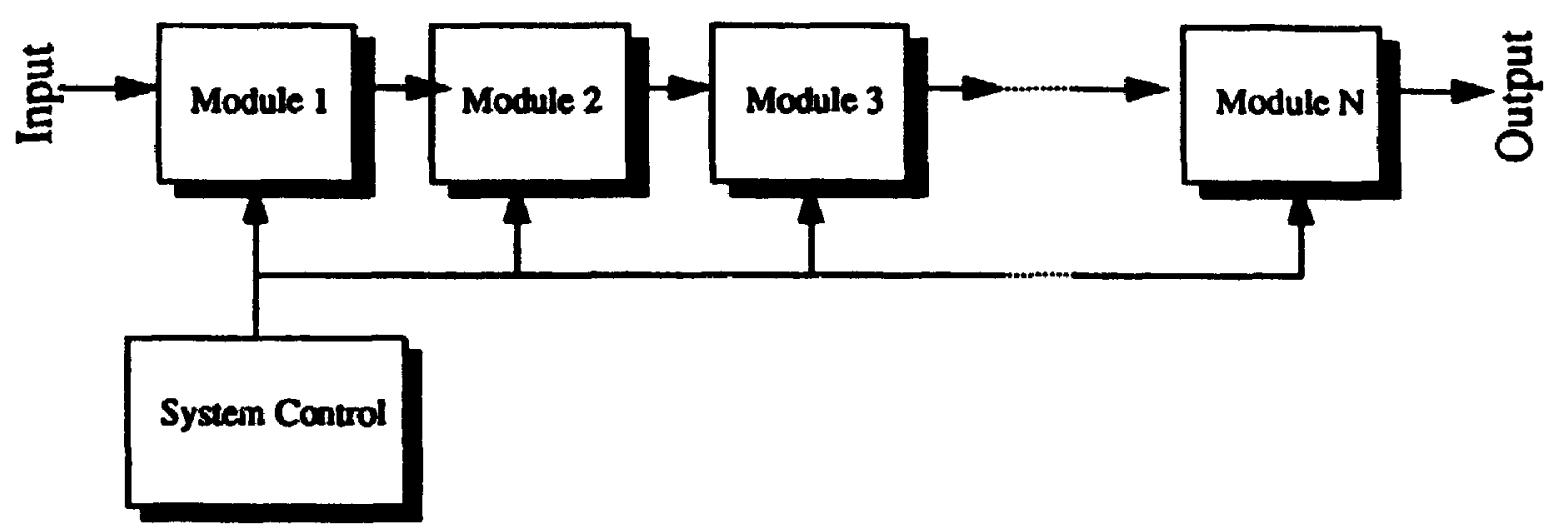

Figure 2.3 A Generic Pipelined Architecture.

The criteria for partitioning depend upon various factors such as characteristics of the algorithms and physical constraints of the hardware implementation. One must carefully select the correct algorithm for a particular application and map the algorithm onto the pipelined architecture. In order to achieve a high throughput rate and low system latency, the functionality of each module must be optimized to take advantage of the characteristic of the pipelined architecture. Although the VLSI implementation technologies are advancing rapidly, there remains an upper limit at which the number of transistors can be packed onto a single silicon substrate. For some FFT applications where a large number of transform points is required, one single IC may not be able to contain all the necessary hardware to complete the transformation. This technological constraint may result in mapping the FFT algorithm onto a multiple chip pipelined 
architecture in order to achieve a reasonable transform size for various digital signal processing applications.

A single pipelined FFT architecture can be implemented by using only two types of processing elements, namely the FFT Switch (Switch) and Complex Butterfly (Butterfly). A block diagram is shown in Figure 2.4. Whereas the FFT Switches, which act like data commutator elements with delay, rout and reorder the correctly sequenced data between the Complex Butterflies, the Butterflies contain all the computational elements and twiddle factors needed to perform numerical calculations of the FFT algorithm.

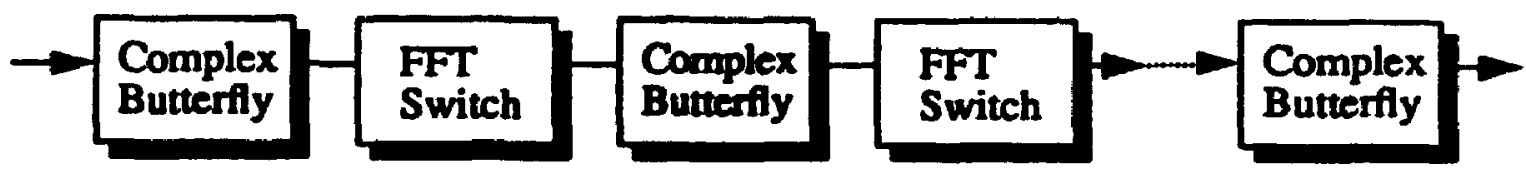

Figure 2.4 A Block Diagram of a Single pipelined Fast Fourier Transform.

There are several forms of FFT algorithms. The radix-2 in-place decimation-intime algorithm was chosen for this project. For a radix-2 FFT implementation, an Npoint pipelined FFT will consist of $\log _{2} N-1$ switching stages and $\log _{2} N$ Complex Butterflies. A 32-point radix-2 forward and inverse pipelined FFT is shown in Figure 2.5. For the forward and inverse pipelined FFTs, the delays $\left\{k_{1}, k_{2}, k_{3}, k_{4}\right\}$ of the Switches correspond to $\{8,4,2,1)$ and $(1,2,4,8\}$ respectively. As for the Complex Butterflies, different numbers of twiddle factors are required for each computational stage in order to achieve a successful transform. Architecturally, the inverse pipelined 
FFT corresponds to a transpose of the forward pipelined FFT [15]; hence the same set of integrated circuits can be arranged to perform both forward and inverse transforms.

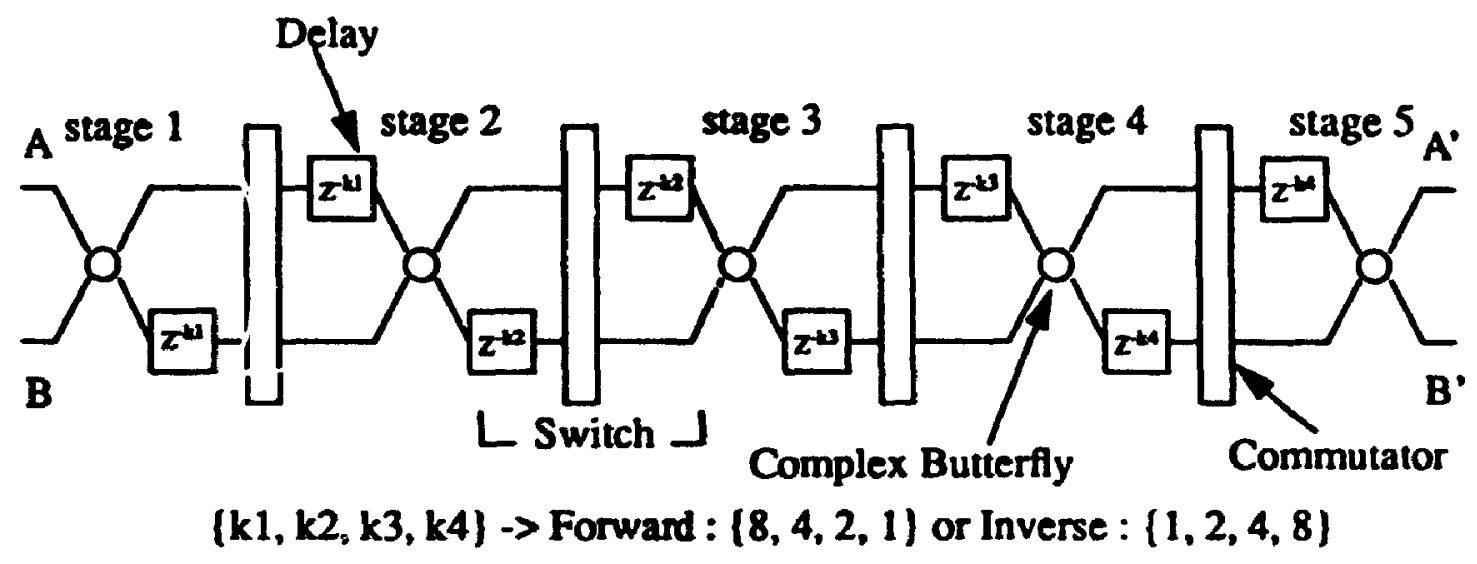

Figure 2.5 A 32-point radix-2 pipelined fast Fourier transform

The signal flow diagram in Figure 2.2 can be mapped directly onto the single pipelined architecture in Figure 2.5, where the arrows and crosses represent the operations of the FFT Switches and Complex Butterflies respectively.

The diagram in Figure 2.5 corresponds to one Butterfly per stage of the pipelined FFT and thus has one degree of parallelism. However, it is possible to reduce the latency of the system by decreasing the total number of pipelined stages and to perform more of the computations in parallel. The parallel pipelined FFT [16] extends the concept of the pipelined FFT and provides an even greater processing capability. A 32-point radix2 parallel pipelined FFT is shown Figure 2.6. No Switches are necessary between the first and second Butterfly of each of the two branches as the interconriections are hardwired. It is possible to further increase the processing speed through the utilization of parallel pipelined architectures with a higher degree of parallelism. 


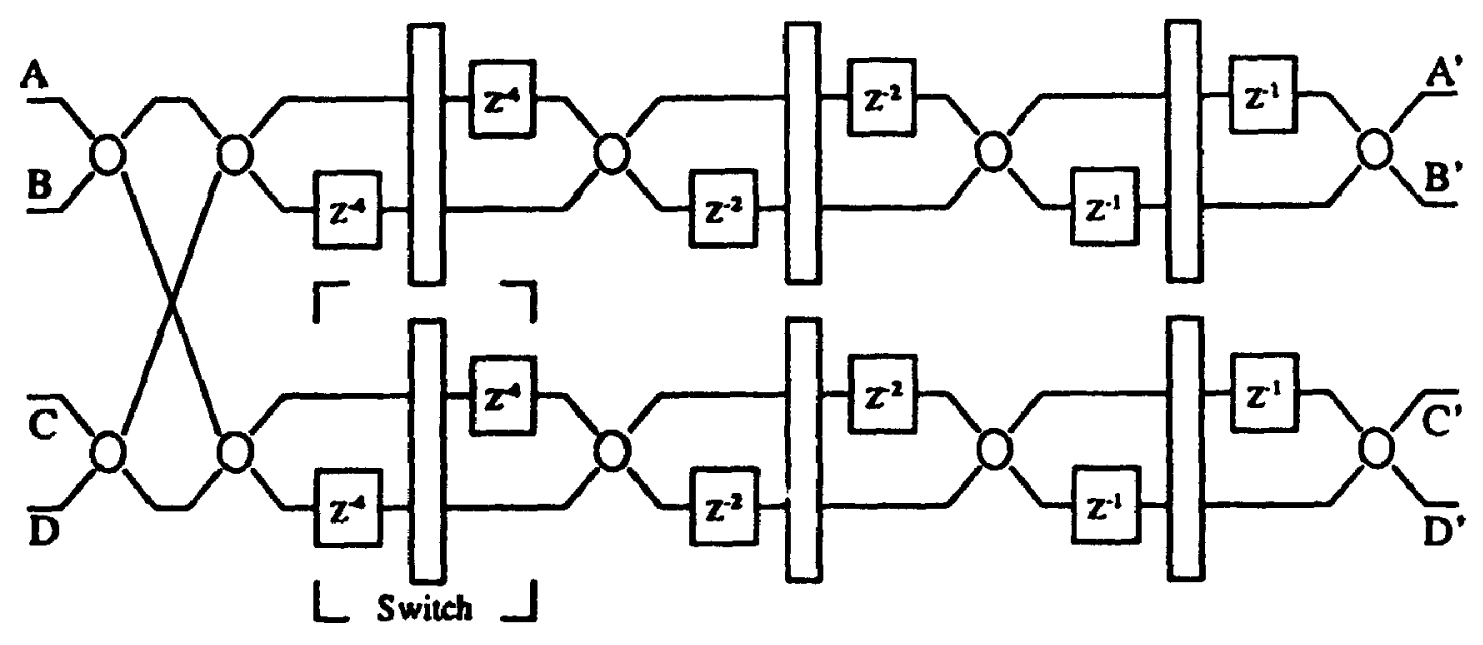

Figure 2.6 A 32-point Radix-2 parallel pipelined fast Fourier transform

In order to achieve a $100 \%$ hardware efficiency for both pipelined and paralle] pipelined implementations, it is necessary to pre-format the input data stream to the parallel inputs of the structure [6][17]. Hardware efficiency is used to measure quantitatively the amount of time (as a percentage) that computational elements are kept busy. Thus for the 32-point single pipelined FFT, the sequential data samples in each 32 sample window must be formatted such that samples $0,1,2, \ldots, 15$ are routed to port $A$ and samples 16, 17, 18, ..., 31 are routed to port B of Figure 2.5. A corresponding timing diagram is shown in Figure 2.7. As for the parallel pipelined 32point FFT, the sequential data samples are formatted such that samples $0-7,16-23,8-15$ and 24-31 are routed to ports A, B, C and D respectively in Figure 2.6. The timing diagram of the parallel pipelined FFI is shown in Figure 2.8. From the timing diagrams, it can be seen that the parallel pipelined architecture is able to compute a complete set of 32 samples in approximately half the number of clock periods required for the single pipelined architecture. 


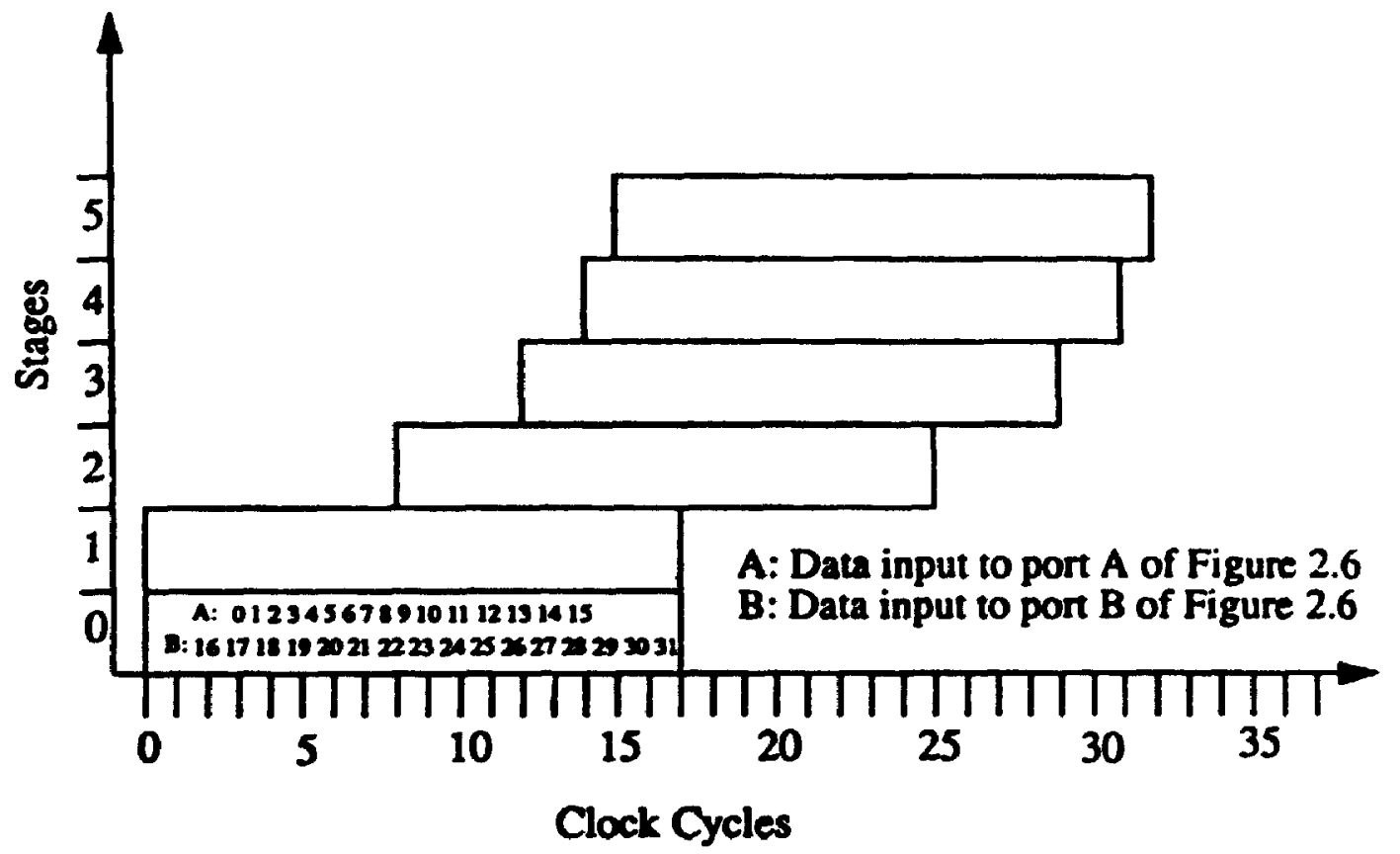

Figure 2.7 A timing diagram of the single pipelined FFT.

\subsubsection{Single Chip Approach}

In order to achieve high speed operation and minimize the chip count of the entire processing system, one must examine the single chip architecture, whose overall power consumption is generally lower than the multichip approach. A number of single chip FFT processors have been reported in the literature [18][19][20]. Each processor contains all the necessary computational, delay memory, coefficient memory, switching and control elements to compute the transform.

Since technology constraints limit the amount of hardware to be implemented on a single processor, the number of transform points for a processor remains low. Subsequently, some processors are implemented with the discrete Fourier transform 


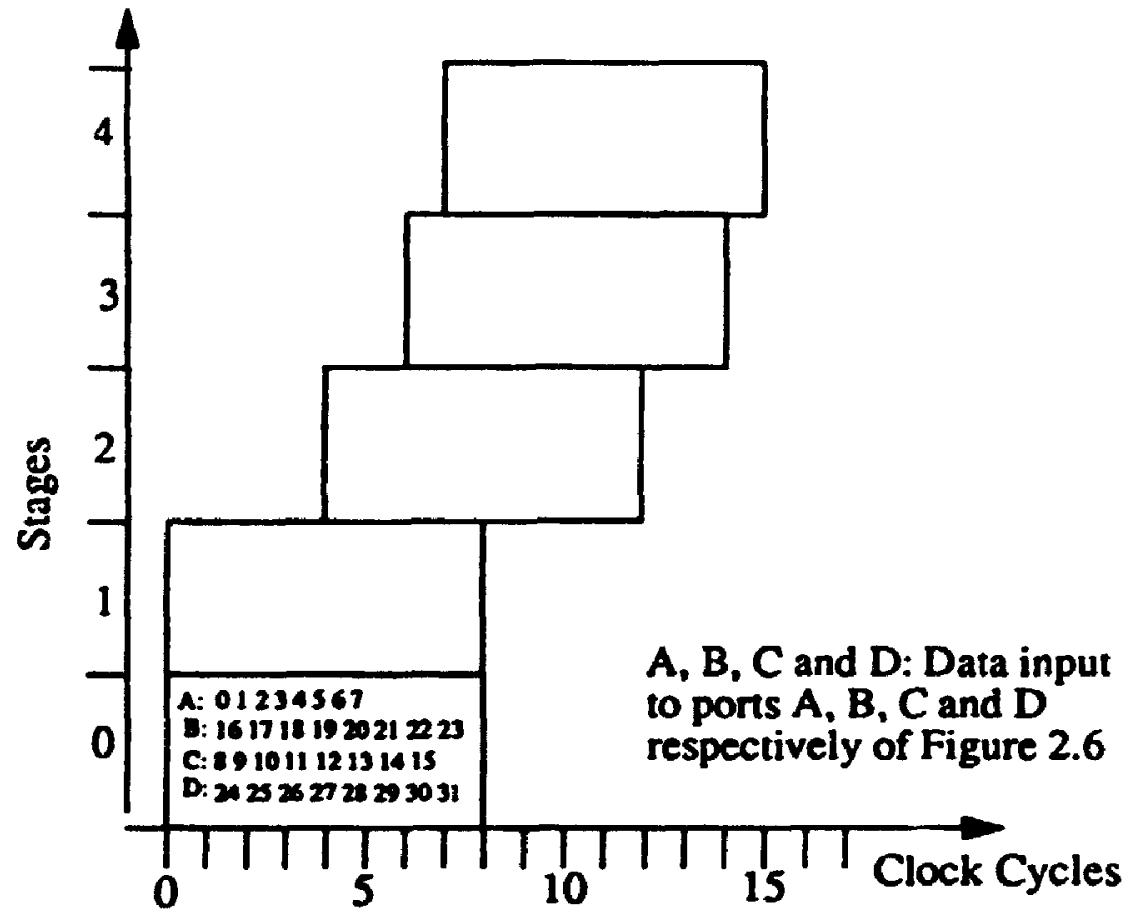

Figure 2.8 A timing diagram of the parallel pipelined FFT.

algorithm rather than the fast Fourier transform algorithm to minimize control overhead. In the case of higher transform sizes, most of the single chip processors are designed such that they are capable of operating in parallel to accomplish, for instance in [19], a 1024-point FFT implementation. However, an excessive amount of external hardware such as multiplier-accumulators and storage memory elements is required in such an implementation. As a result, the eventual size and cost of the system increases a great deal.

\subsubsection{Multichip Approach}

For many FFT applications where extremely high throughput is essential, multichip architecture may be the only feasible option to achieve performance 
requirements. In fact, the multichip processor implementations are popular and have been reported in the literature [21]-[26] and more recently some chip sets have become available commercially [33][34]. Pipelined architecture is used to increase throughput. As mentioned in section 2.3.3.2, a pipelined processor can be implemented using only two building blocks, namely an FFT Switch and a Complex Butterfly. It is possible to achieve a spectrum of transform sizes by cascading a number of the two building blocks with minimal external circuitry required.

More recently, due to the advances in packaging technology, researchers have been able to further improve system performance by introducing techniques such as the Wafer-Scale integration (WSI) [25][26] and the Multichip Module (MCM) [31]|32]. These techniques allow the reduction of the propagation delay (due to smaller interconnection parasitics) between each integrated circuit. Traditionally, a solution which employs a printed circuit board with off-the-shelf components and custom-built VLSI circuits is used. However as the clock frequency and data throughput increase, data transfer between ICs on the printed circuit board becomes a problem. Although the very expensive ECL and GaAs technologies (at the time of writing) can provide the necessary density and speed required for many digital signal processing applications, the requirement of high power consumption will be marked as a severe constraint for some systems. Hence, both WSI and MCM become very attractive alternatives for digital signal processing hardware implementations. Nevertheless, several architectural problems exists inciuding testability and power consumption, which require more thorough examination before either can be fully realized. 


\subsection{Summary}

The multi-carrier demodulator for digital signal processing on-board of a satellite consists of a demultiplexer, one of the techniques to construct a demultiplexer is to use a polyphase filter bank cascaded with a FFT processor. A number of architectural alternatives can be used to implement the processor. However, in order to achieve the 25 MSPS per channel performance specification within a reasonable size and cost, the multichip pipelined architecture approach is adopted in this project. The initial requirement of a 32-point radix-2 processor can be realized by using a chip set consisting of a FFT Switch and a Complex Butterfly. In the next chapter, the architectural considerations of the FFT processor for our application are discussed. 


\section{Chapter Three}

\section{Architectural developments for the Pipelined Fast Fourier Transform Chip Set}

\subsection{Introduction}

The design of the pipelined FFT chip set is based upon a number of architectural considerations which reflect both technology constraints and certain performance and system level requirements. These considerations will be discussed for the FFT Switch and briefly for the Complex Butterfly.

The chip set implements the decimation-in-time (DIT) radix-2 algorithm for high performance fast Fourier transform applications. The operations of the FFT algorithm are partitioned such that it is possible to obtain maximum flexibility. Both Switch and Butterfly are implemented in CMOS Gate Array tochnology. 


\subsection{Problems Definition}

As mentioned in chapter one, the 25 MSPS per channel radix-2 32-point FFT processor is required as part of a demultiplexer in a multi-carrier demodulator for onboard digital signal processing. In order to achieve the system throughput requirement, a multichip pipeline approach is adopted. The chip set consists of two generic building blocks namely, an FFT Switch and a Complex Butterfly operating in a pipelined architecture as shown in Figure 2.4. Although the initial requirement for the demultiplexer is a 32-psint FFT processor, it would be desirable to implement processors of higher transform sizes for other applications using the same chip set. In order to achieve this objective, the FFr Switch must be capable of providing the necessary number of delays and the correct reordering and routing operations between Complex Butterflies. Since a limited number of delny elements can be implemented on a single Switch, it is necessary for the Switch to be operated in a chain (cascadeable). In addition, the Complex Butterflies must be able to access the complex coefficients (twiddle factors) efficiently for the numerical computations.

In order to ease the increasingly more difficult task of testing an integrated circuit (IC), both chips should include some testability features. As the number of transform points grows, the number of ICs in a pipeline is expected to increase. Hence, an effective technique must be implemented such that the overall system control overhead is minimized, and as a result, the complexity of the system control circuitry is reduced. These issues which concern the design of the chip set are addressed in the next few sections. Although the architectural problems of the chip set have been identified, the solutions will be limited by technological constraints, such as total gate count, 
component library, device speed, power consumption and so on, of the available gate array family.

\subsection{Architectural Considerations for the FFT Switch}

\subsubsection{Introduction}

The basic function of the FFT Switch is to provide the necessary clock delays and to reorder and rout the data between the Complex Butterflies. Routing is different from reordering in the sense that it represents a fixed data path in the commutator. A block diagram of the Switch is shown in Figure 3.1. Detailed circuit descriptions of all the functional blocks will be included in the next chapter. However, architectural issues such as cascadeability, control and testability measures which influence the design of the Switch will be discussed in the next few sections.

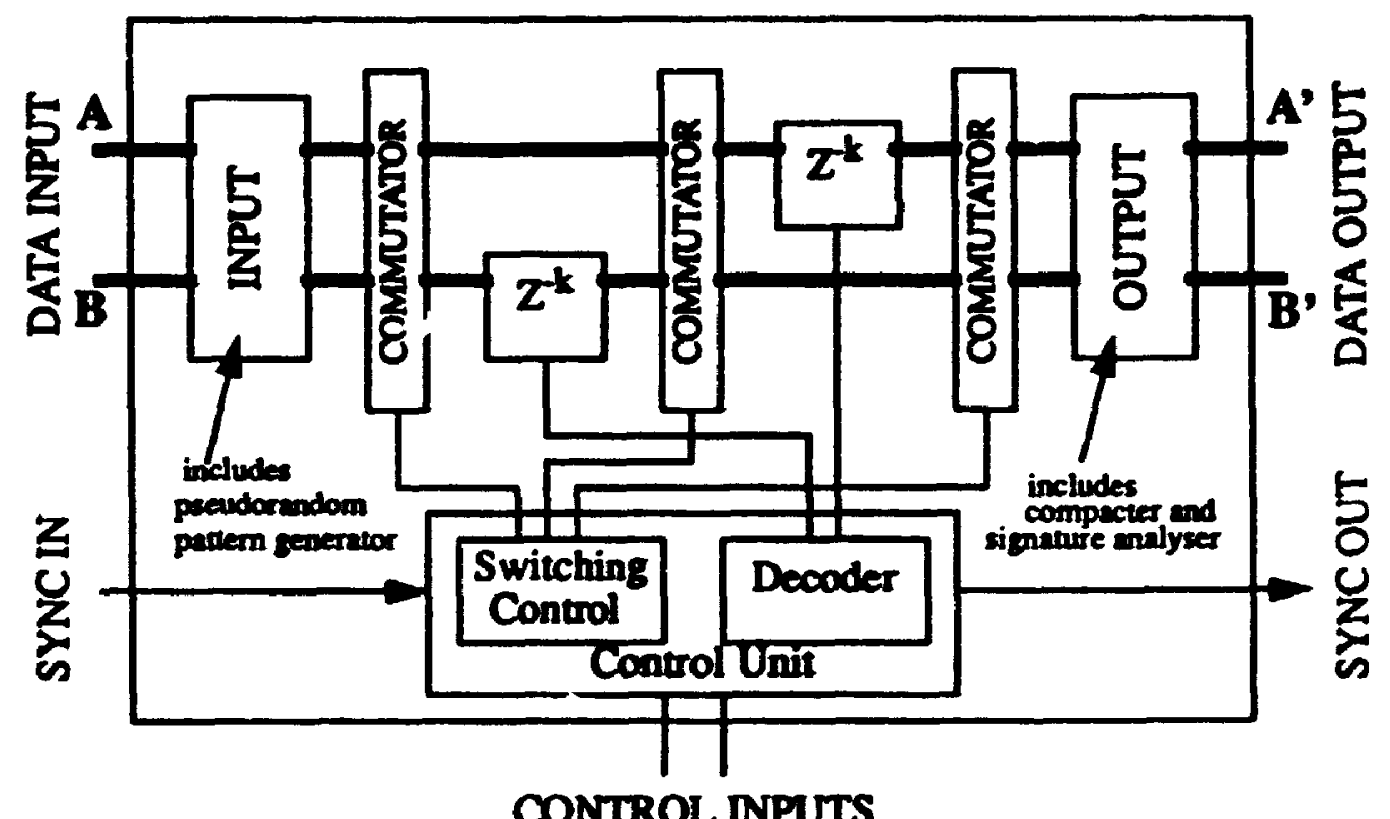

Figure 3.1 A block diagram of the FFT Switch 


\subsubsection{Cascadeability}

The amount of storage required by a Switch in an $\mathrm{N}$-point pipelined $\mathrm{FFT}$ depends upon the value of $\mathrm{N}$ and the position of the Switch within the pipeline. As the value of $\mathrm{N}$ increases, the number of stcrage elements required to implement the clock delays will increase. Furthermore, the exact position of the Switch within a pipeline is vital in determining the necessary number of clock delays before and after the reordering of data. Both technologically imposed limitations on the Switch storage capacity and the requiremınt for a single generic Switch capable of supporting large FFT sizes dictate that the Switch must be cascadeable. A generic Switch implies that the module must be capable of implementing all the switching functions in a pipelined FFT architecture. In order to accomplish the cascadeability requirement, a threecommutator architecture is adopted for the Switch (see Figure 3.1). The commutators are used to rout or to reorder the data through the Switch. All three commutators are identical and their select inpuis are controlled by signals which originate from the "Control Unit". The select signals determine the operation of the commutators and subsequently a number of modes can be implemented by the Switch. There are all together five separate modes of operation as shown in Figure 3.2. In order to keep the generic nature of the Switch, every Switch must be capable of operating in all five modes.

Only one Switch is responsible for the reordering of data when a number of Switches are cascaded. This particular Switch is named, appropriately, the Principle Switch. The rest of the Switches on both sides of the Principle Switch provide the necessary delay for the pipeline. The Principle Switch is represented by the Middle and End switch modes depicted in Figure 3.2. As their names imply, the Middle and End 


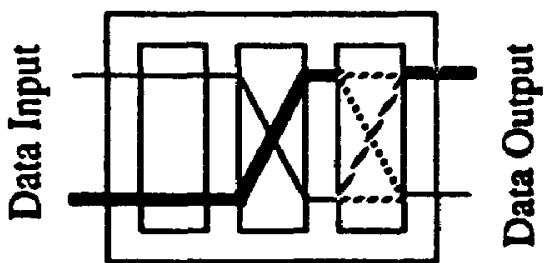

End

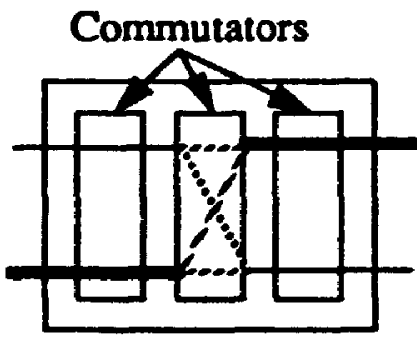

Middle

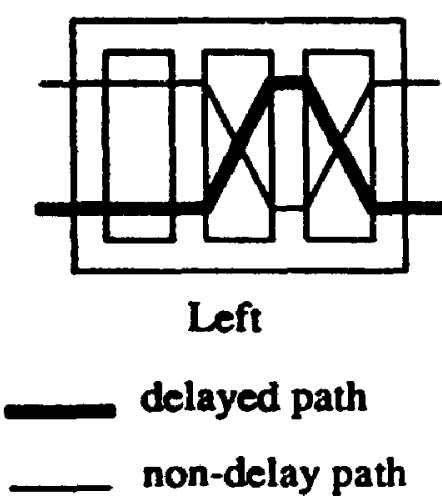

non-delay path

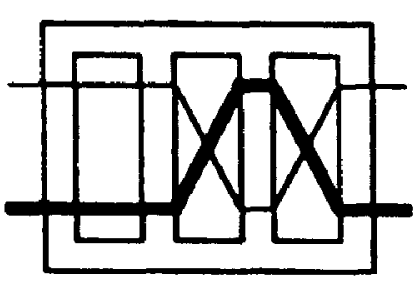

Right

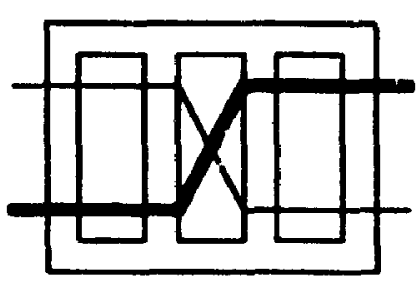

Output

Figure 3.2 Five modes of operations

switch modes correspond to the cases where the second (i.e. middle) and third (i.e. end) of the three commutators of the Principle Switch reorder the data. In cases where a number of Switches are cascaded, those to the left and right of the Principle Switch operate in the Left and Right switch mades respectively. Operation in the two switch modes differs only in the manner in which the synchronization pulse is processed. In all instances where two or more Switches are cascaded, the last Switch must operate as the Output switch mode to ensure data is routed correctly before it proceeds to the next Butterfly stage.

A block diagram of a 128 point radix-2 pipelin. d FFT processor is shown in Figure 3.3, and Figure 3.4 shows the first set of the cascaded Switches in a 128-point radix-2 pipelined FFT where all the commutators in the Switches are included. Since a 


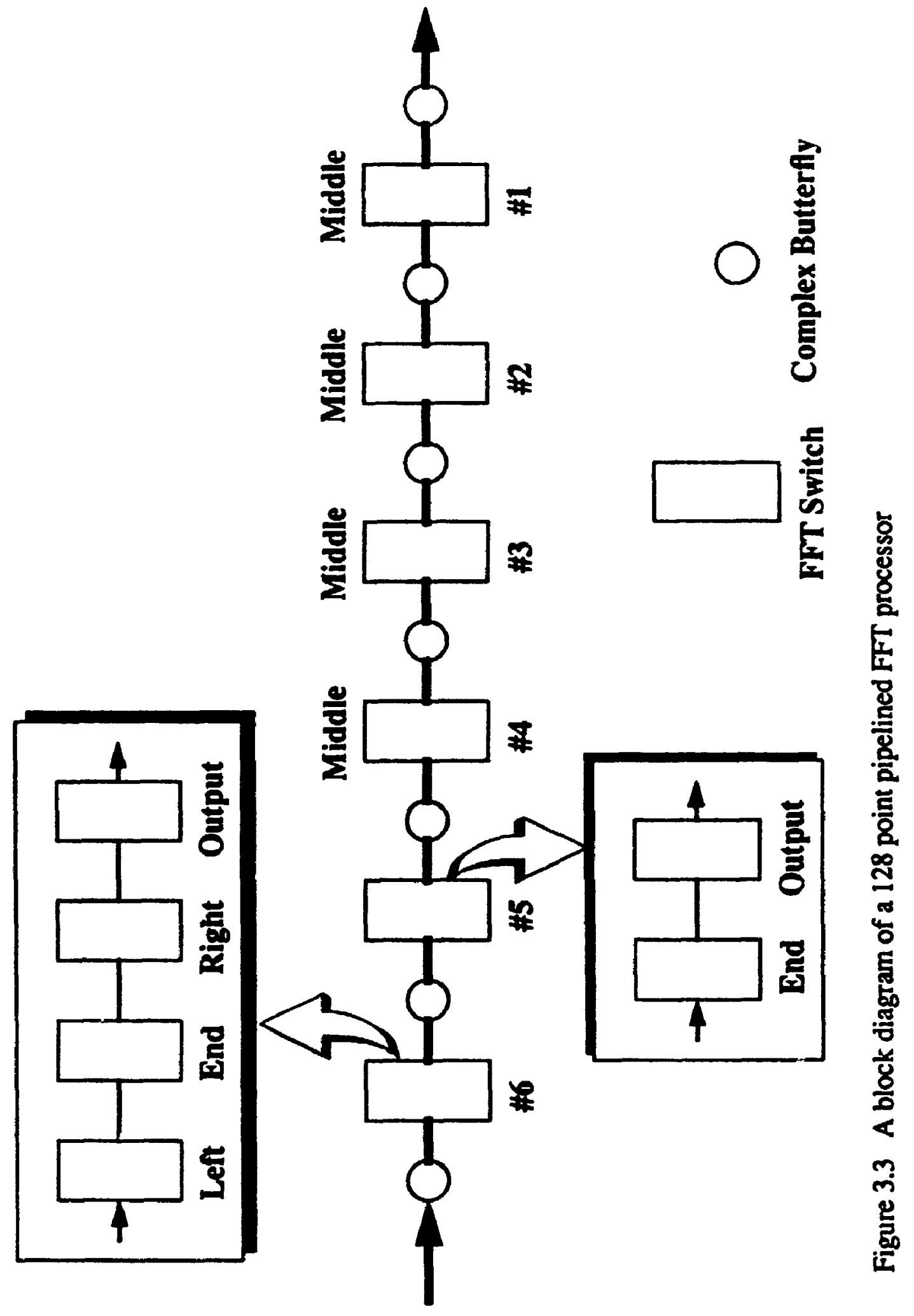


maximum of eight clock cycle delays per channel is possible for each Switch and a total delay of 32 clock cycles is required for this particular stage (stage \#6), four Switches are necessary for the cascaded structure. The thick lines represent the delay path of the cascaded structure. All data will pass through the same number of delay elements and hence data at output $A^{\prime}$ and $B^{\prime}$ is once again aligned before the next processing stage. In this instance, the Principle Switch is operated at the second module of the structure.

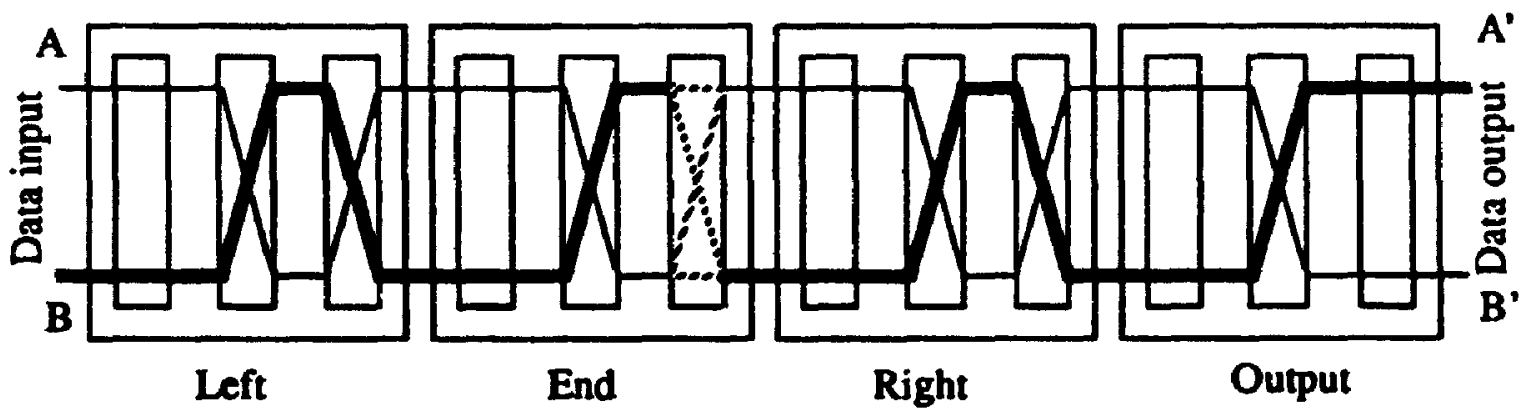

Figure 3.4 Four cascaded Switches

\subsubsection{Switch Control}

Architecturally, both the Switch and the Butterfly are aimed at minimizing control overheads on the system level for the pipelined FFT. The proposed processor architecture should adopt a distributed control methodology where almost all control functions are generated on-board the Switches and Butterflies. The necessary information will be loaded into the modules only once and the control signals will be generated accordingly. Information must include the location of the Switch relative to the whole pipeline and the switch mode of each module. 
In order to identify and to ensure the synchronization of valid data in the pipeline, the synchronization (Sync) pulse technique is proposed. The Sync pulse will be generated by the system control of the pipelined processor and propagated along with the data. A negative going edge indicates the start of the valid data and initiates the reordering process. The Sync pulse will remain active low as long as the input data is valid and terminates the reordering process when the Sync pulse returns to active high. Figure 3.5 illustrates how valid data is defined by the Sync pulse. Same number of delay clock cycles will be imposed on both data and Sync pulse in every stage of the processor.

Data input

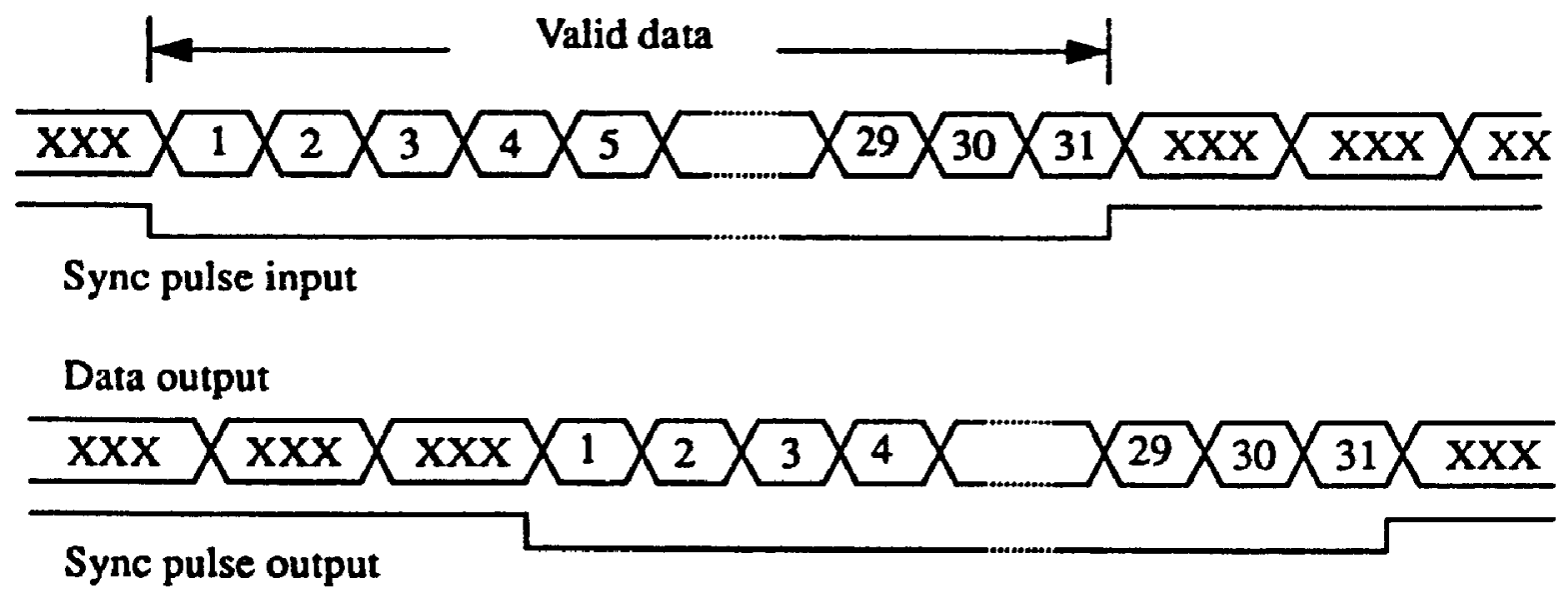

Figure 3.5 A diagram to illustrate the operation of the Sync pulse.

As the number of cascaded modules increases in a pipelined FFT processor, power consumption for the overall system becomes a critical issue. Consequently, it is most desirable to disable those portions of the Switch which are not used. Since some of the delay elements contained in the Switches located at the last few stages, preceding 
the pipeline output are not required at all, they are automatically disabled to reduce power consumption. More registers will be disabled in a parallel pipelined architecture where there are more unused registers in the structure.

\subsubsection{Testability Issues}

Although functional testing traditionally constitutes the basic test strategy for integrated circuits, the cost of functional testing is becoming excessively expensive due to the rapid increase in circuit density and pin count. Subsequently, the automatic test equipment (ATE) used to complete functional testing is considered either too costly or inadequate to accomplish the task. Test pattern generation also requires (particularly for complex circuits) long computation. It appears that the most attractive solution to reduce testing expenses is to include circuitry on each chip to factilitate testing. The test approach adopted for both the FFT Switch and Complex Butterfly is the built-in selftest (BIST) technique based on the generation of pseudorandom test vectors to provide the stimulus of the circuit and analyses the results at the output by using response compression[27][28]. The response compression technique is used to reduce the volume of output data by first allowing the response pattern to pass through a circuit called a compacter which reduces the number of bits after a data compression is performed. A compacted output is compared with a predetermined test response known as the signature. Generally, a single bit pass/fail output will define the status of the circuit. Figure 3.6 shows a block diagram of the BIST arrangement. All BIST procedures are performed separately from normal operations, hence the "off-line" test method. Since testing is accomplished "off-line", there are no constraints imposed on the operating frequency to complete the testing procedures. As a result, testing can be done at design speed or higher. 


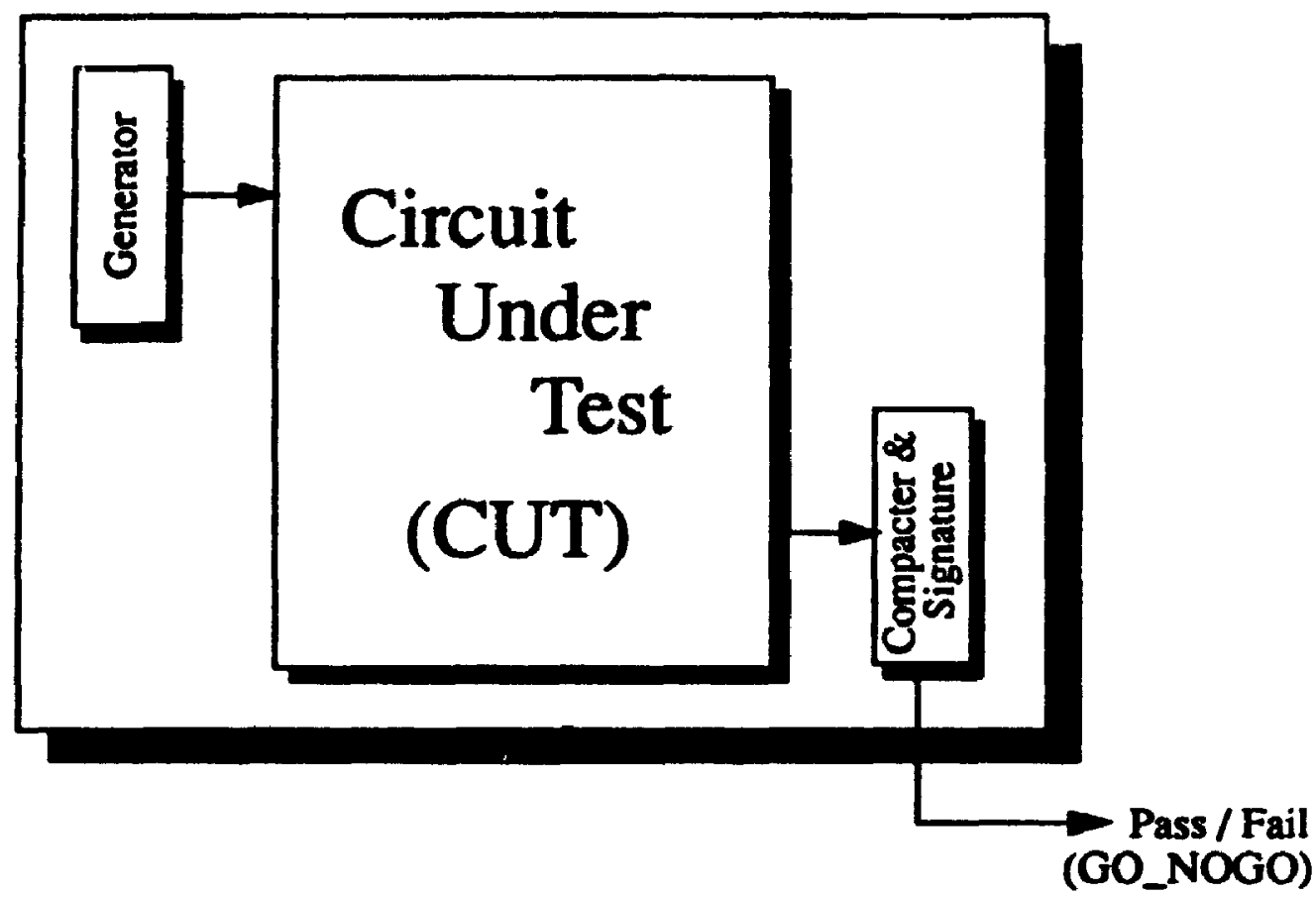

Figure 3.6 A built-in self-test arrangement.

The disadvantage of BIST technique is that extra circuit area is required to accommodate testing and ofien BIST related performance degradation of the circuit also occurrs. Although there is no strict guideline on the percentage of circuitry in an IC dedicated to testing, normally every effort is made to minimize it.

In essence, the main motivation in pursuing the BIST approach has been to reduce the cost of testing, and this technique has proved to be very popular. Even with the disadvantages mentioned above, the benefits of the BIST make it a worthwhile technique. 


\subsection{Architectural Considerations for the Complex Butterfly}

In this section, a brief account of some design considerations for the Complex Butterfly will be presented. A more thorough discussion of the Butterfly implementation is included in the literature [22]. The Butterfly was designed in advance of the FFT Switch. A block diagram of the Butterfly is shown in Figure 3.7.

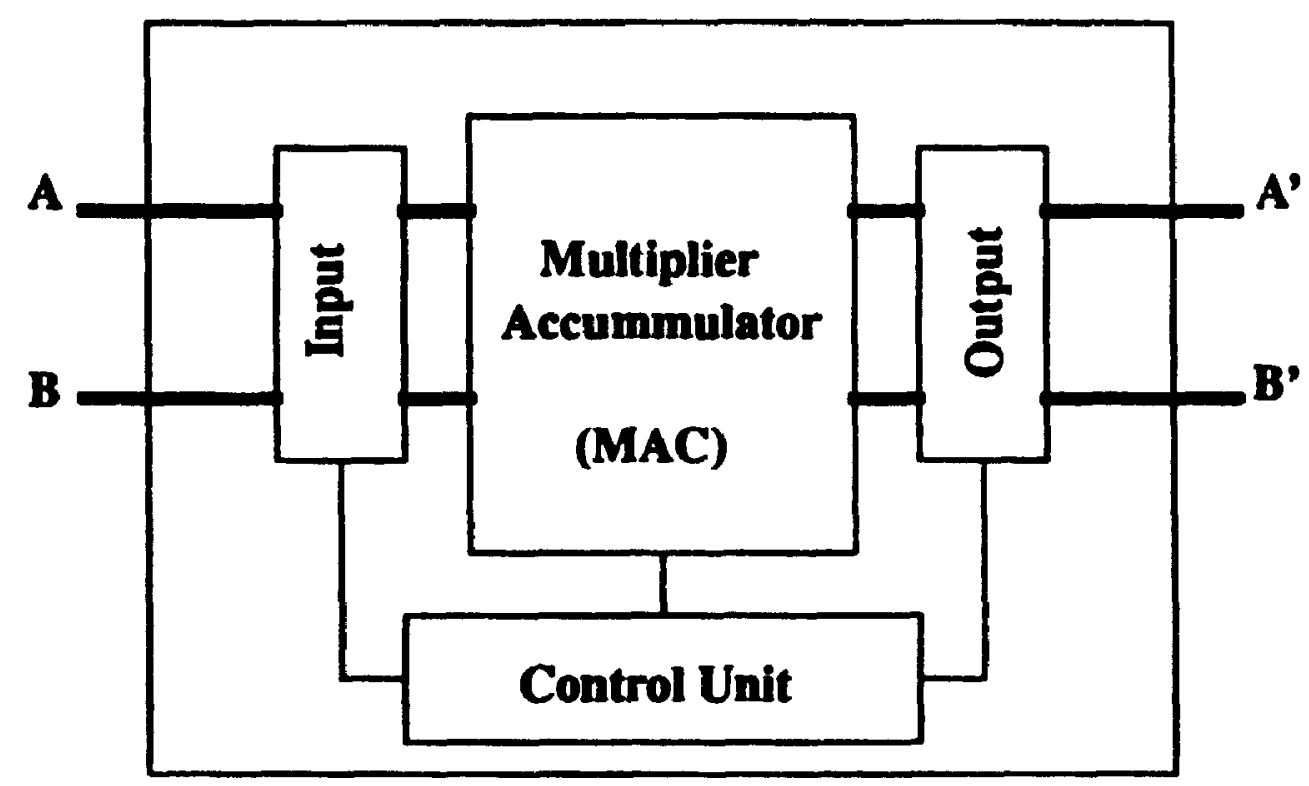

Figure 3.7 A Block Diagram of a Complex Butterfly

The decimation-in-time FFT algorithm implemented by the Butterfly can be expressed by Equation 3.1-3.4

$$
a^{\prime}=a+(c \times \cos \theta-d \times \sin \theta)
$$




$$
\begin{aligned}
& c^{\prime}=a-(c \times \cos \theta-d \times \sin \theta) \\
& b^{\prime}=b+(c \times \sin \theta+d \times \cos \theta) \\
& d^{\prime}=b-(c \times \sin \theta+d \times \cos \theta)
\end{aligned}
$$

where $A=a+j b$ and $B=c+j d$ represent the complex inputs and $A^{\prime}=a^{\prime}+j b^{\prime}$ and $B^{\prime}=c^{\prime}+j d^{\prime}$ represent the complex outputs of Figure 3.7. In order to achieve a throughput of 25MSPS per channel system requirement, multiple-levels of pipelining have to be incorporated into the architecture of the multiplier accumulator of the Complex Butterfly. Techniques such as the modified Booth Algorithm, Wallace Adder Tree and Redundant Binary Arithmetic are implemented in a synchronous pipelined architecture. Since there is always a limit for the number of transistors to be implemented on a single die, it is possible that only a relatively small number of twiddle factors can be stored on-board the Complex Butterfly. The solution is such that the Butterfly can either read the necessary twiddle factors stored in on-board memory or be loaded with the twiddle factors from an external RAM or ROM in a sequential manner. Since not all butterfly stages require a large number of different twiddle factors, they are loaded into the Butterfly and can be accessed in a cyclic fashion. Similarly, the Butterfly circuit also accommodates the distributed control and test methodology to minimize control overheads on the system level.

\subsection{System Control}

Since most control signals are generated within the FFT Switches and Complex Butterflies, external interference to the flow of data during normal operation will be kept at a minimum. Nevertheless, the system control must be capabie of distinguishing the validity of the data and producing the correct Sync pulse signal accordingly. 
Furthermore, the system control will supervise the loading of information from an external source to all the modules in the pipeline, apart from the more general distributed signals such as clock pulse and clear pulse.

\subsection{Functional Simulation of the Pipelined FFT}

\subsubsection{Introduction}

Prior to, the development of a detailed Switch design at the schematic level, a functional simulation of a 32-point radix-2 single pipelined FFr is performed. Functional simulation at a high level of abstraction enables one to gain a better understanding of the overall system. The modified Splice is used as the simulator. Splice is a circuit and logic simulator which can handle multiple levels of abstraction from transient analysis of MOS and bipolar integrated circuits to functional block models. The block models are implemented as a sequence of code based on their arithmetic or logical functions. The models will be assumed as basic primitives for simulations. All functional models are written in "Ratfor" which is a FORTRAN preprocessor language. The models' code, will be compiled and linked with the simulator as subroutines, and can be used like any other Splice source deck. Both FFT Switch and Complex Butterfly models use the electrical analysis mode. The electrical analysis is chosen to obtain detailed analog voltage waveforms for the circuit blocks under analysis. Using the analog representation simplifies the interconnects betwee:! models by replacing 4 sets of 16 bit digital connections with 4 analog connections and consequently reduces the evaluation time. 


\subsubsection{Models for the FFT Switch and the Complex Butterfly}

A 32-point radix-2 pipelined FFT model can be implemented by using four FFT Switch models and five Complex Bunerfly models. Figure 3.8 and Figure 3.9 show block diagrams of the Switch and the Butterfly models as they are implemented in Splice. The four analog connections represent the real and imaginary parts of the two complex data channels

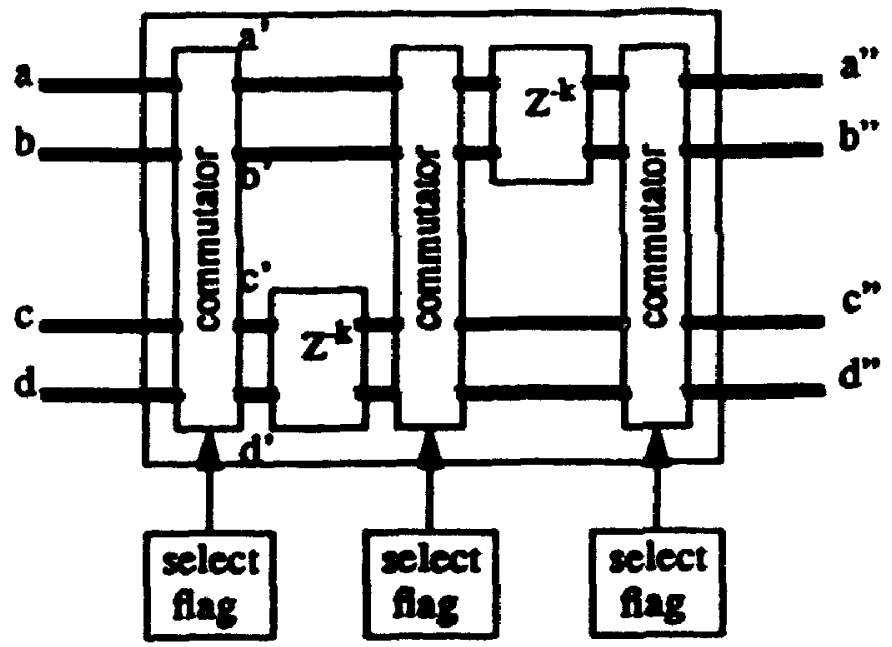

Figure 3.8 A model for FFT Switch.

The Switch is modelled as three commutators with delays in between. The delay is represented by $Z^{-k}$ blocks in the Figure 3.8 . The commutators are controlled by separate select flags. The operation of the commutator is shown in Figure 3.10.

The input to each commutmor either passes straight across or crisscrosses, depending on the select flag. For a 32-point pipelined FFT implementation, only the centre commutator of all the Switches is required for data reordering. Figure 3.11 and 


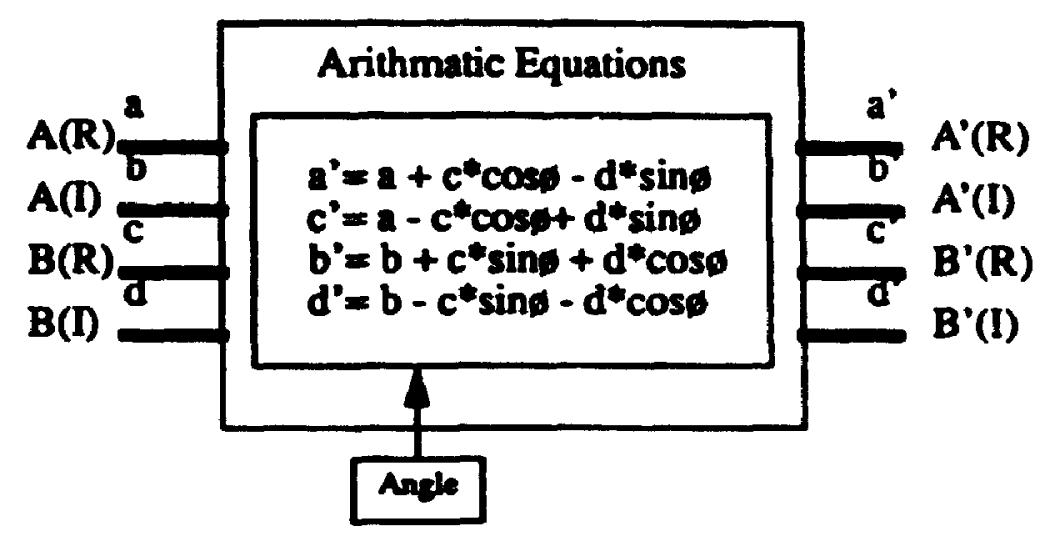

Figure 3.9 A model for the Complex Butterfly.

select

$$
\begin{array}{lllll}
1 & a \rightarrow a^{\prime} & b \rightarrow b^{\prime} & c \rightarrow c^{\prime} & d \rightarrow d^{\prime} \\
0 & a \rightarrow c^{\prime} & b \rightarrow d^{\prime} & c \rightarrow a^{\prime} & d \rightarrow b^{\prime}
\end{array}
$$

Figure 3.10 Operations of the commutator

Figure 3.12 shows the Ratfor code for the delay element and commutator respectively in Ratfor.

The arithmetic unit in the butterfly implements the DIT algorithm with equations shown in Equations 3.1-3.4. Since the Splice source desk is written in Ratfor, the Sine and Cosine functions in the equations can be determined with reasonable precision. Twiddle factors for the equations are generated in a different block called the Angle block, separately from the Complex Butterfiy model. Only a small number of twiddle factors in each stage is required to compute the 32-point pipelined FFT and these are 


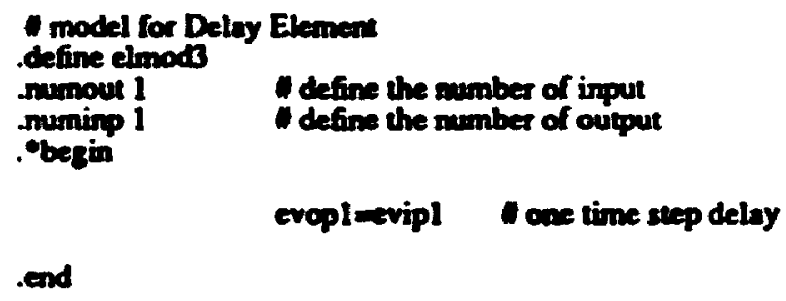

Figure 3.11 Model code for the Delay Element

accessed in a cyclic manner. The Ratfor code for the Complex Buttertly and Angle models is shown in Figure 3.13 and Figure 3.14 respectively.

\subsubsection{Simulation Results}

It is possible to examine the functionality of a pipelined forward FFT structure by using a Sine wave as the time-domain input. Since the mapping of a correctly sampled Sine wave in the time-domnin to frequency-domain is a periodic impulse train located at the fundamental frequency and its harmonic, it will be a simple task to verify the functionality of the system. Figure 3.15 and Figure 3.16 show some of the simulation results which verify the system functionally.

\subsection{Summary}

An FFT processor can be implemented by using a chip set which consists of an FFT Switch and a Complex Butterfly. The two building blocks must be cascadeable in order to realize a pipelined stnucture. Furthermore, due to technological constraints, the Switch is designed such that it can be cascaded with another Switch. Cascadeable 


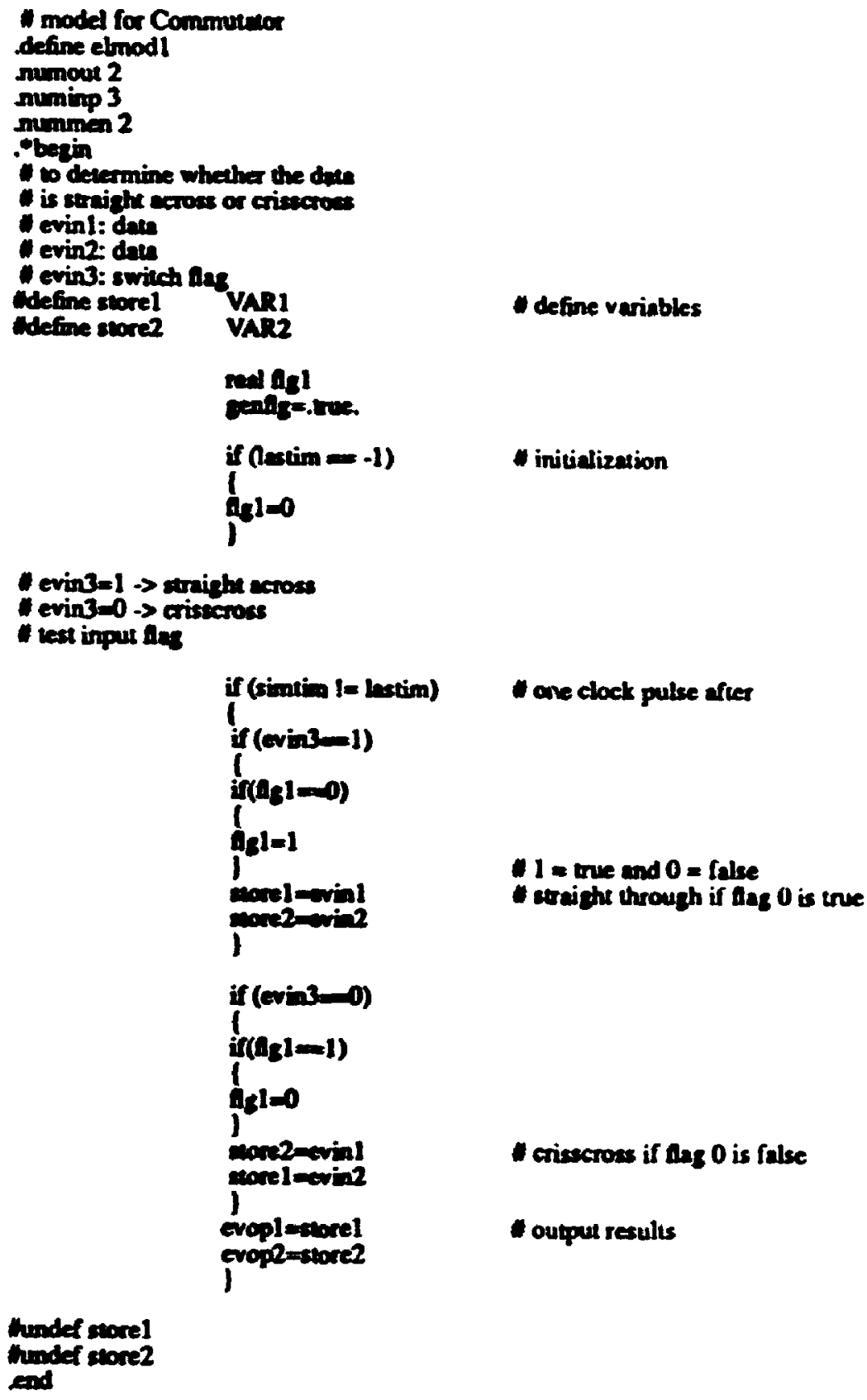

Figure 3.12 Model code for the Commutator 


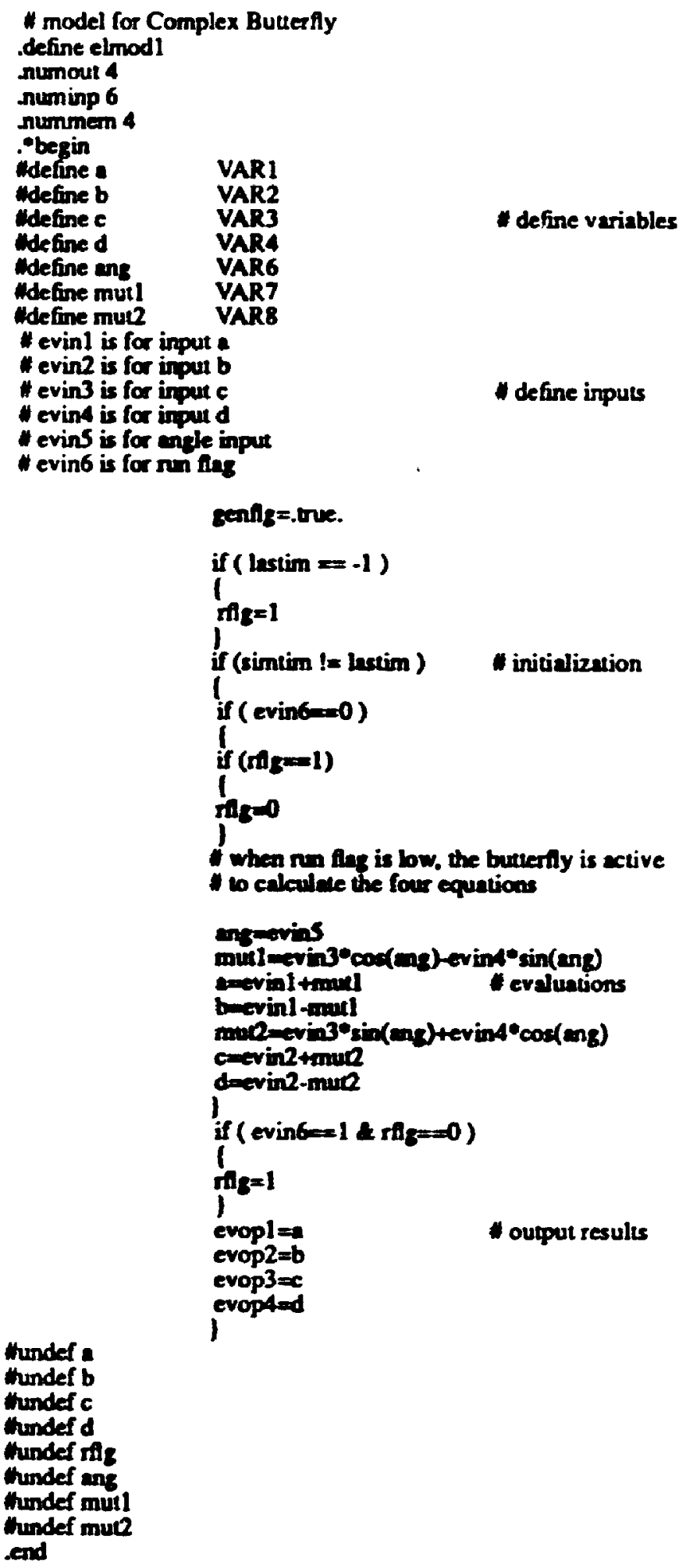

Figure 3.13 Model code for the Complex Butterfly 


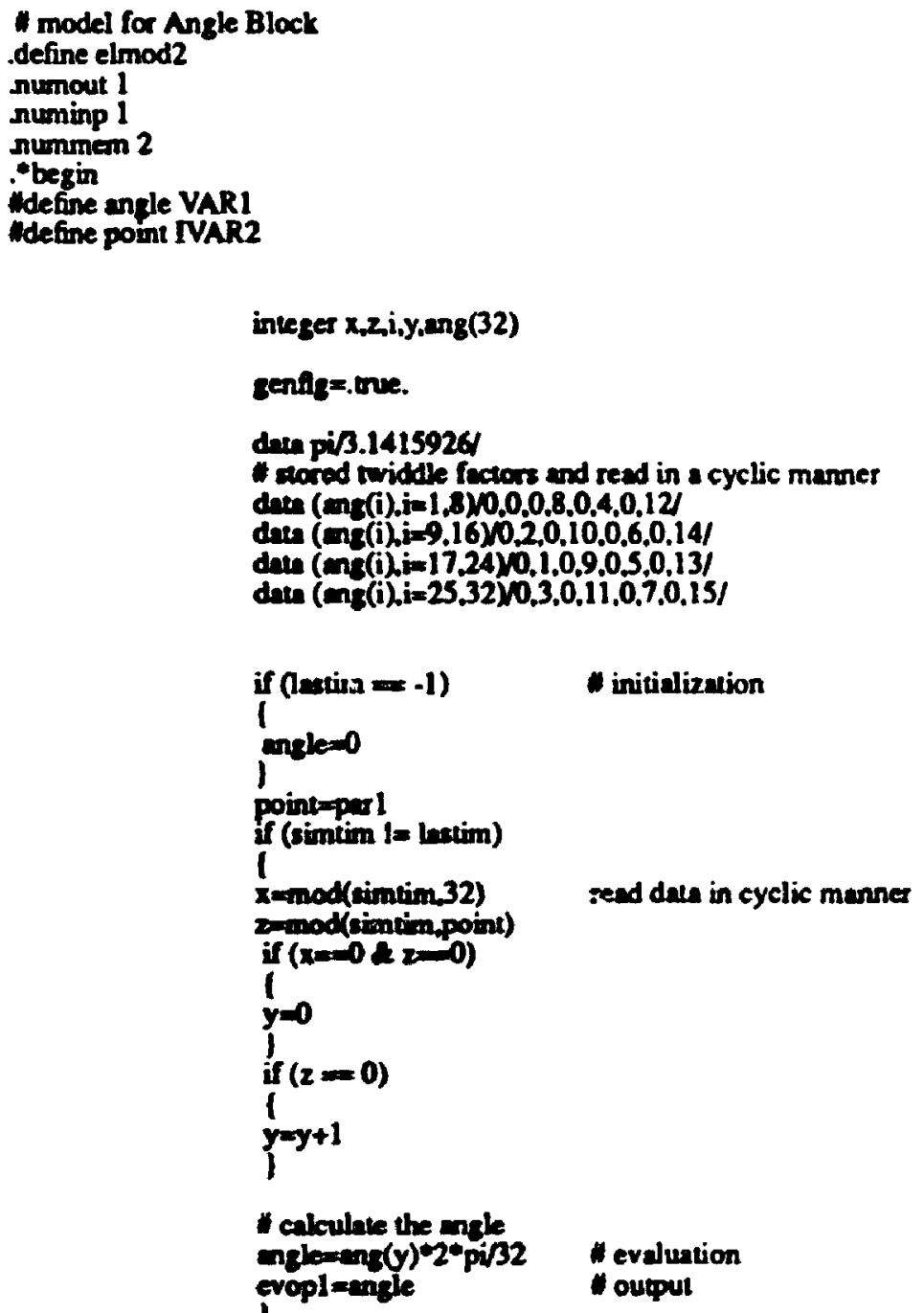

Tundef angle

Hundef point

Figure 3.14 Model code for the Angle Block

Switches are necessaiy to increase the number of delays per channel so that it is possible to implement other pipelines of larger transform sizes. Both the Switch and the Butterfly adopt a distributed control and test methodology in order to minimize the system control overheads. The most attractive strategy to reduce the expense of IC testing is to 


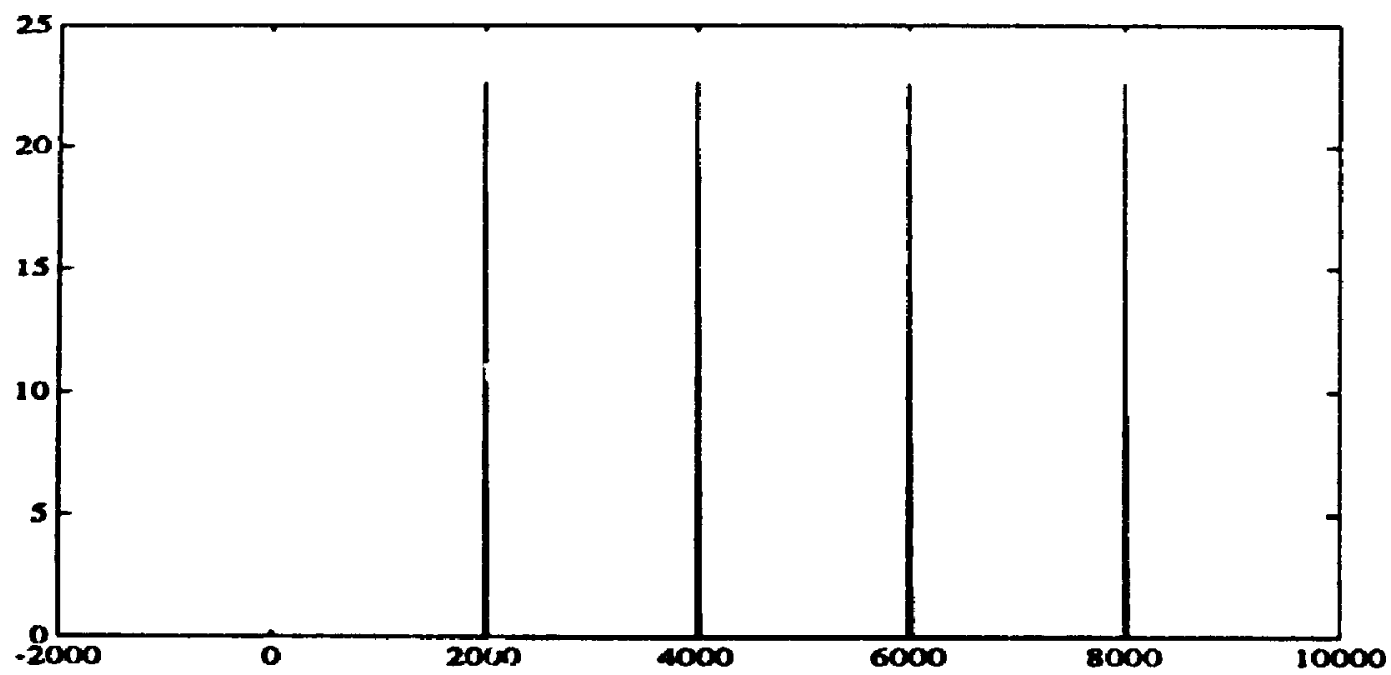

Figure 3.15 The simulated Splice output with a $2 \mathrm{KHz}$ input source

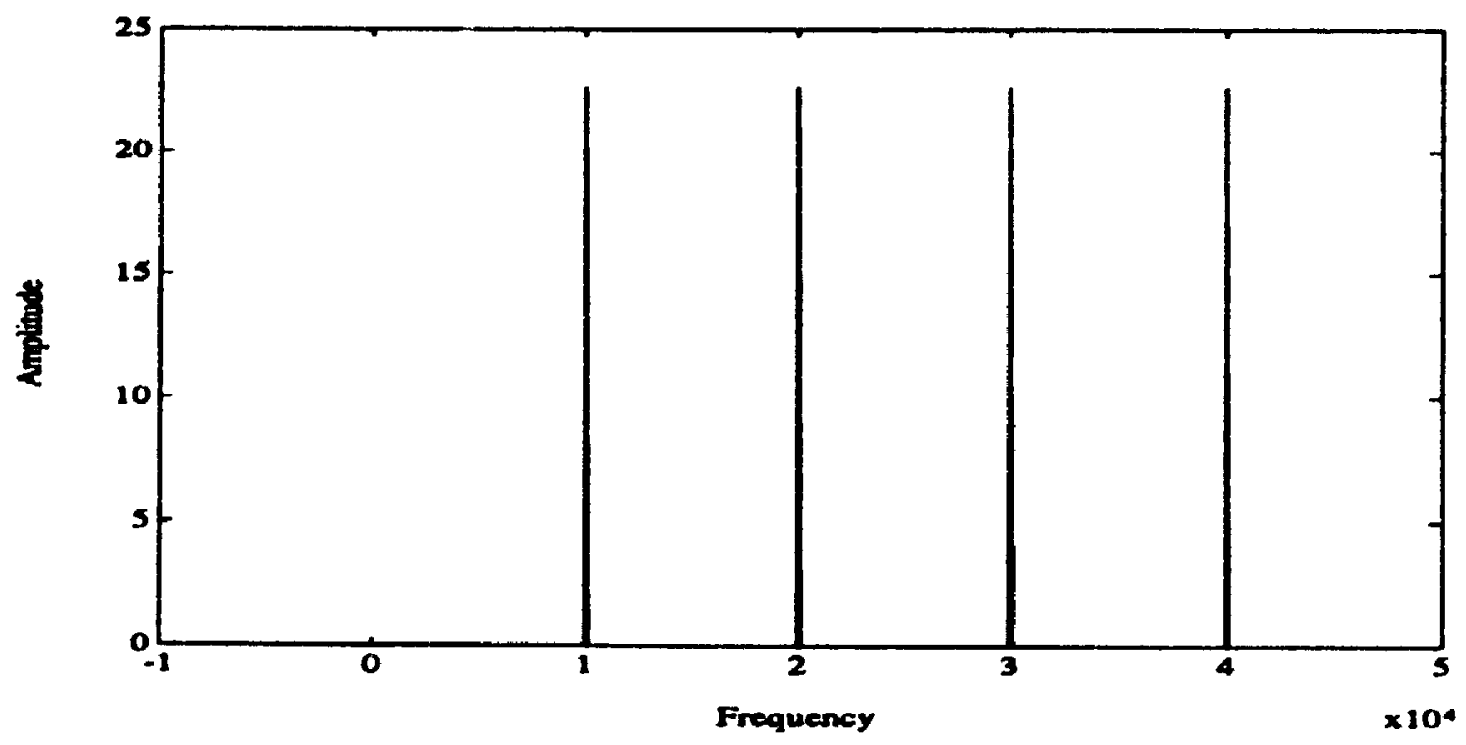

Figure 3.16 The simulated Splice output with a $10 \mathrm{KHz}$ input source 
include circuitry on the chip to factilitate testing. As described earlier, one of the selftest techniques is based on the generation of pseudorandom test vectors as the stimuli of the circuit and it analyses the result at the output by using response compaction. This built-in self-test technique is inplemented in both the Switch and the Butterfly. A Splice simulation of the pipelined FFT has been performed and the functionality of the model is verified. In the next chapter, the detailed circuit description of the FFT Switch is presented. 


\section{Chapter Four}

\section{Circuit Description of the FFT Switch}

\subsection{Introduction}

The architectural considerations of the pipelined FFT described in chapter three clearly indicate the directions for the FFT Switch implementation. Before the detailed circuit description of FFT Switch is presented, a brief account of Computer Aided Design (CAD) tools, which are used in the design of the Switch, will be given. The design is implemented by using the CMOS Gate Array technology to achieve shorter design cycles and lower fabrication cost over a full custom design, which occurs at the expense of higher gate count and hence higher power consumption. Circuit description of the Switch will be partitioned into sections which include the delay element and commutator, control unit, input/output blocks and circuit for testability. Simulations of the FFT Switch will be presented in the following chapter. 


\subsection{Computer Aided Design Tools}

\subsubsection{Introduction}

Since the traditional "breadboard" prototype techniques are impractical for complex I.C. designs, alternative design methodologies are required. The fact IC designers are facing the need for increasingly more complex designs and shorter design cycles further spells the importance of Computer Aided Design (CAD) tools. The introduction of the use of advanced CAD tools has vastly reduced design cycle time and improved the quality of products. By using such tools, designers are able to put together circuits and systems in a much quicker, more accurate and more convenient manner than ever before. CAD tools allow designers to capture the schematic of the circuit and simulate the design thoroughly. Extensive simulation helps shorten the total development time by reducing the number of prototype iterations. Designs remain in the iteration loop, which consists of schematic capture refinements and accurate circuit simulations, until they have fully met performance specifications. Finally, the design of the integrated circuit is expanded ${ }^{1}$ and the netlist extracted. The layout of the circuit must be completed before the design is sent to the foundry. All CAD tools used in the FFT Switch design are supplied by Mentor Graphics and run on version 10 of the Aegis $^{2}$ Operating System.

\subsubsection{Schematic Capture Tools}

In order to perform hierarchical IC designs, a circuit editor $\left(\right.$ NETED $\left.^{3}\right)$ and a symbol editor $\left(\mathrm{SYMED}^{2}\right)$ is used. These tools allow a designer to create a schematic

1. A process which maps a hierarchical design of a circuit into a "one-level" design in order to extract the netlist of the circuit.

2. Trademark of HP/Apollo.

3. Trademarks of the Mentor Graphics. 
sheet at a lower hierarchical level by using NETED, which can be represented by a symbol block, generated in SYMED, for a schematic sheet at a higher hierarchical level. Consequently, the complexity of the schematic at high hierarchical levels will be reduced and visual verification by the designer can be simplified. The component library used throughout the design of the FFT Switch is based on the LSI Logic LMA $9 \mathrm{~K}$ library. It is a $1.5 \mu \mathrm{m}$ HCMOS Gate Array technology and the available device family has a maximum gate utilization of 15,000 .

\subsubsection{Simulation Tools}

To analyse and verify an electronic design created in the schematic capture tools, a logic simulator (QUICKSIM ${ }^{l}$ ) and a fault simulator (QUICKFAULT ${ }^{l}$ ) are used. QUICKSIM is an interactive logic simulator that allows designers to verify the functionality of designs produced with NETED and SYMED. With the logic simulator, designers are allowed to supply stimulus to the designs, run the simulation, analyse the results, and modify the design based on those results. Designers can then re-apply stimulus to the designs and start the cycle again for further simulations. QUICKFAULT is a fault simulator which models manufacturing defects by using the stuck-at fault models. The design is first of all simulated under fault-free condition. Then faults are inserted one at a time before the design is simulated again using the same set of test vectors. Results of the fault and fault-free simulations are compared and subsequently the simulator predicts the fault coverage of the test vectors set.

1. Trademarks of Mentor Graphics 


\subsection{Design Implementation}

\subsubsection{Design of the Delay Circuit and Commutator blocks}

The two delay blocks ( $\mathrm{Z}^{-\mathrm{k}}$ blocks) and the three commutators in Figure 4.1 collectively occupy the majority of circuit area on the FFT Switch. The delay elements are required in the pipelined FFT for the data realignment. Realignment is necessary for

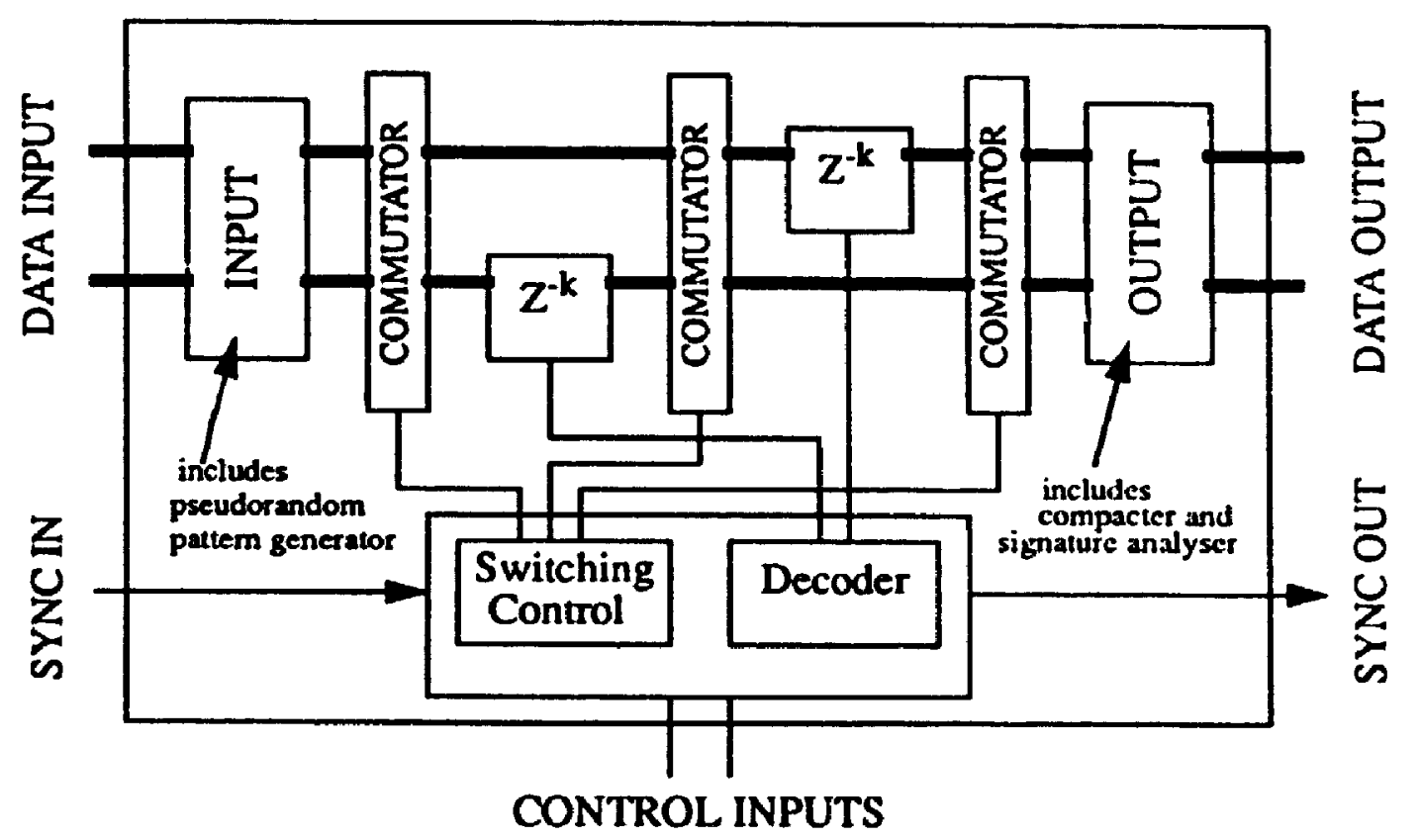

Figure 4.1 A block diagram of a FFT Switch

the data reordering process and is performed before and after the reordering. All delay elements in the $Z^{-k}$ blocks are implemented by means of shift registers. The delay path in each $Z^{-k}$ block is eight delay elements long. For a 32 bit wide data path, an array of $32 * 8$ shift registers is needed. A Random Access Memory (RAM) structure, which can be implemented as the delay element by simply displacing the read and write addresses with a constant value, probably consumes less power than the shift register array. 
However, due to the limited number of library components available and a more complex addressing logic for the RAM structure, a shift register array is chosen to implement the $\mathrm{Z}^{-\mathrm{k}}$ blocks. Figure 4.2 shows a delay line circuit diagram for two bits in the same $\mathrm{Z}^{-k}$ block. In order to provide different delays to compute the transform, the outputs of the first, second, fourth and eighth registers in each row of the delay element are tapped and fed into a four to one multiplexer. A maximum of eight delay clock cycles are possible per bit in each $Z^{-k}$ block. The multiplexer selects the correct number of delays based on the decoder outputs which originate from the "Control Unit ". Both $\mathrm{Z}^{-\mathrm{k}}$ blocks in the same Switch are arranged to provide the identical delay. However it is not necessary for data in both paths to pass through any of the delay blocks. For example, a simplified block diagram of a Switch operating in the Left Switch mode is shown in Figure 4.3. Each $Z^{-k}$ block represents an eight clock cycles delay. Whereas

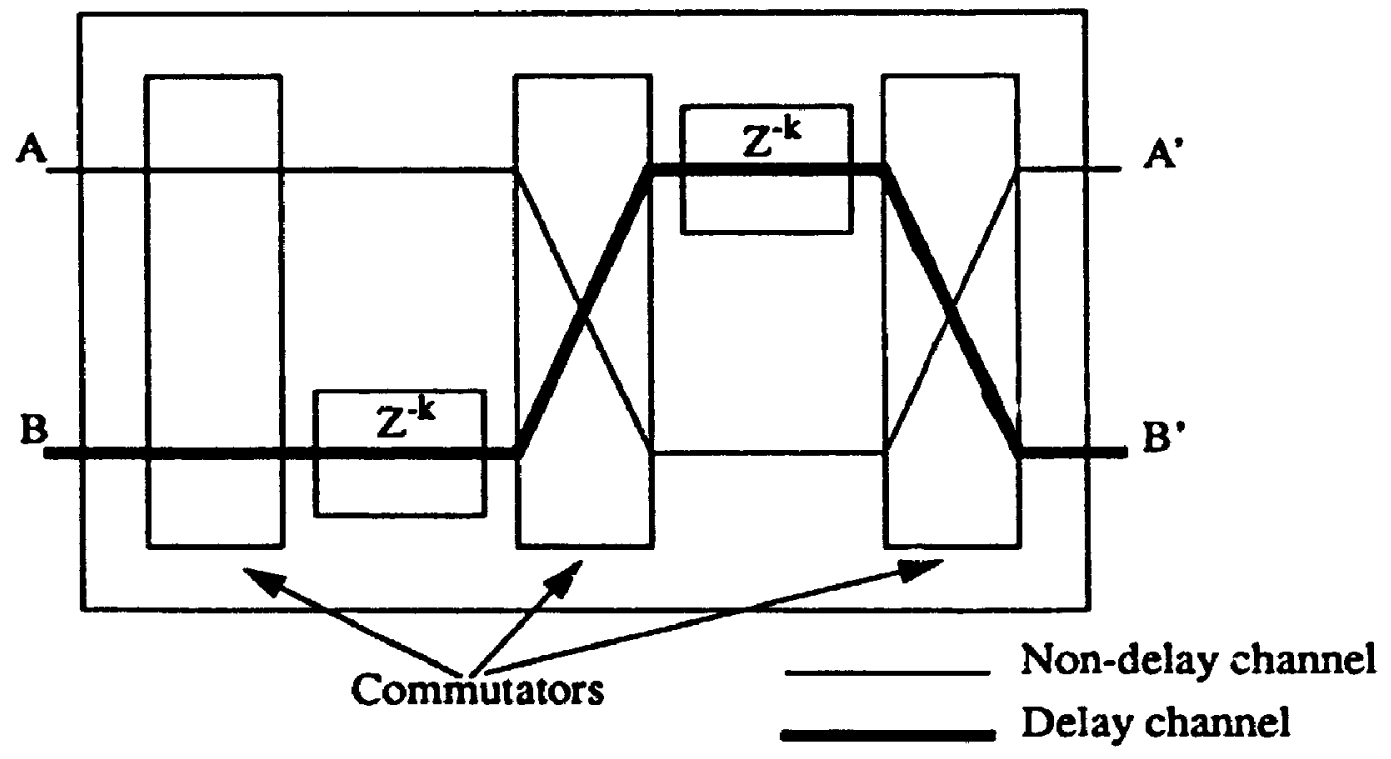

Figure 4.3 Data channels of Left Switch mode 


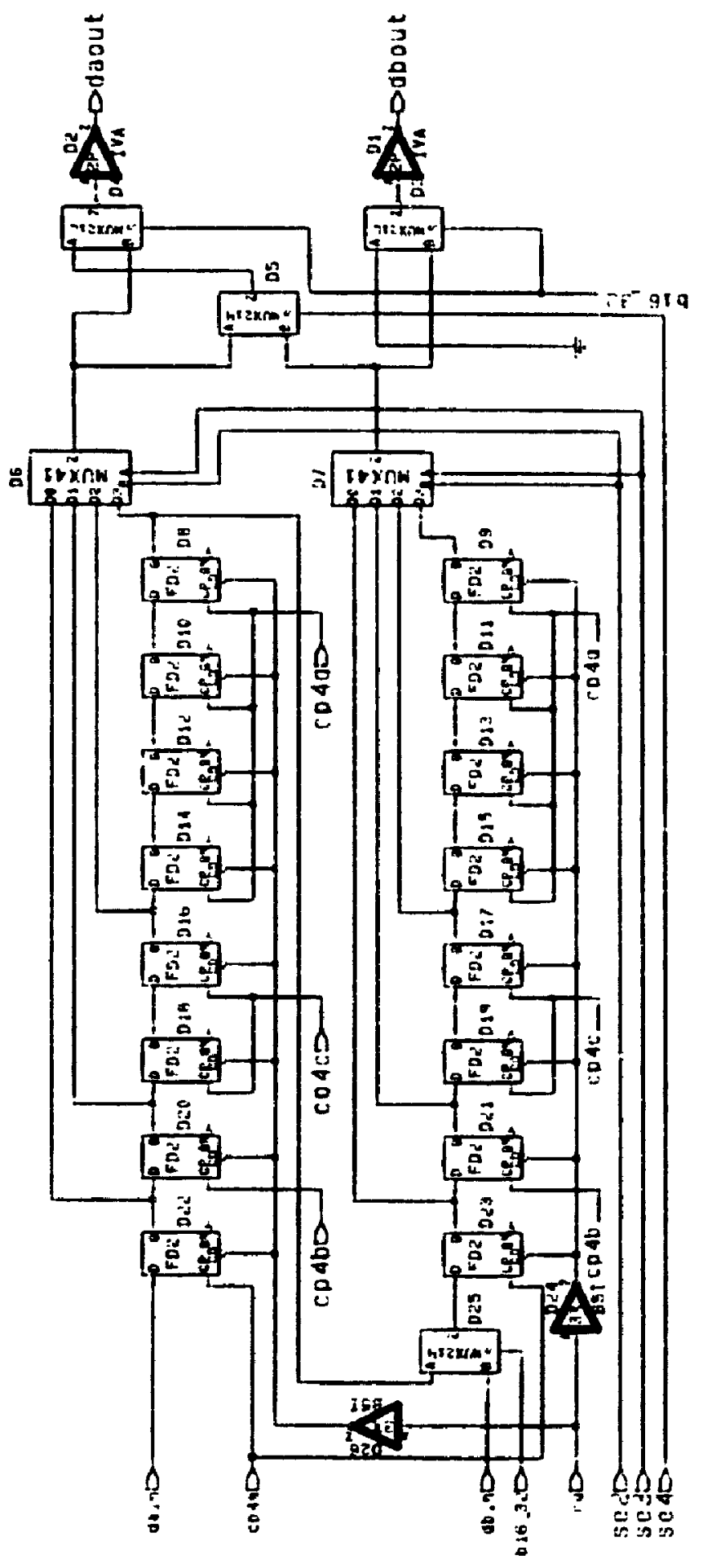

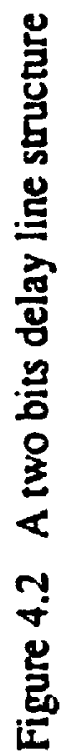


data on channel A does not pass through any delay element at all, data on channel B propagates through both $\mathrm{Z}^{-k}$ blocks, hence a total of sixteen clock cycles delay will be imposed.

In Figure 4.2, the delay line structure is controlled by four separate clock cycles, namely CP4a, CP4b, CP4c and CP4d. All clock cycles originate from the "Control Unit". The four clock cycles approach is employed so that it is possible to distribute the load onto several clock cycle generators and to disable parts of the shift register array by disabling the clock inputs.

Data routing is accomplished by using three separate commutators in each FFT Switch. Their locations are shown in the block diagram of Figure 4.1. The select inputs of all three commutators are also controlled by signals originated from the Control Unit. Each commutator is made up of four sets of multiplexers each set consisting of sixteen two-to-one multiplexers. In order to rout two complex data paths through the commutator, the four sets of multiplexers are arranged as shown in Figure 4.4. Two sets of multiplexers are responsible for routing the real parts of the complex data and the other two sets are associated with the imaginary parts.

Data reordering is done by using either the second or third commutator of the Principle Switch. If a particular commutator is responsible for data reordering, the select inputs for the commutator will be controlled by a periodic square wave called the Reordering Frequency, also generated in the Control Unit. 


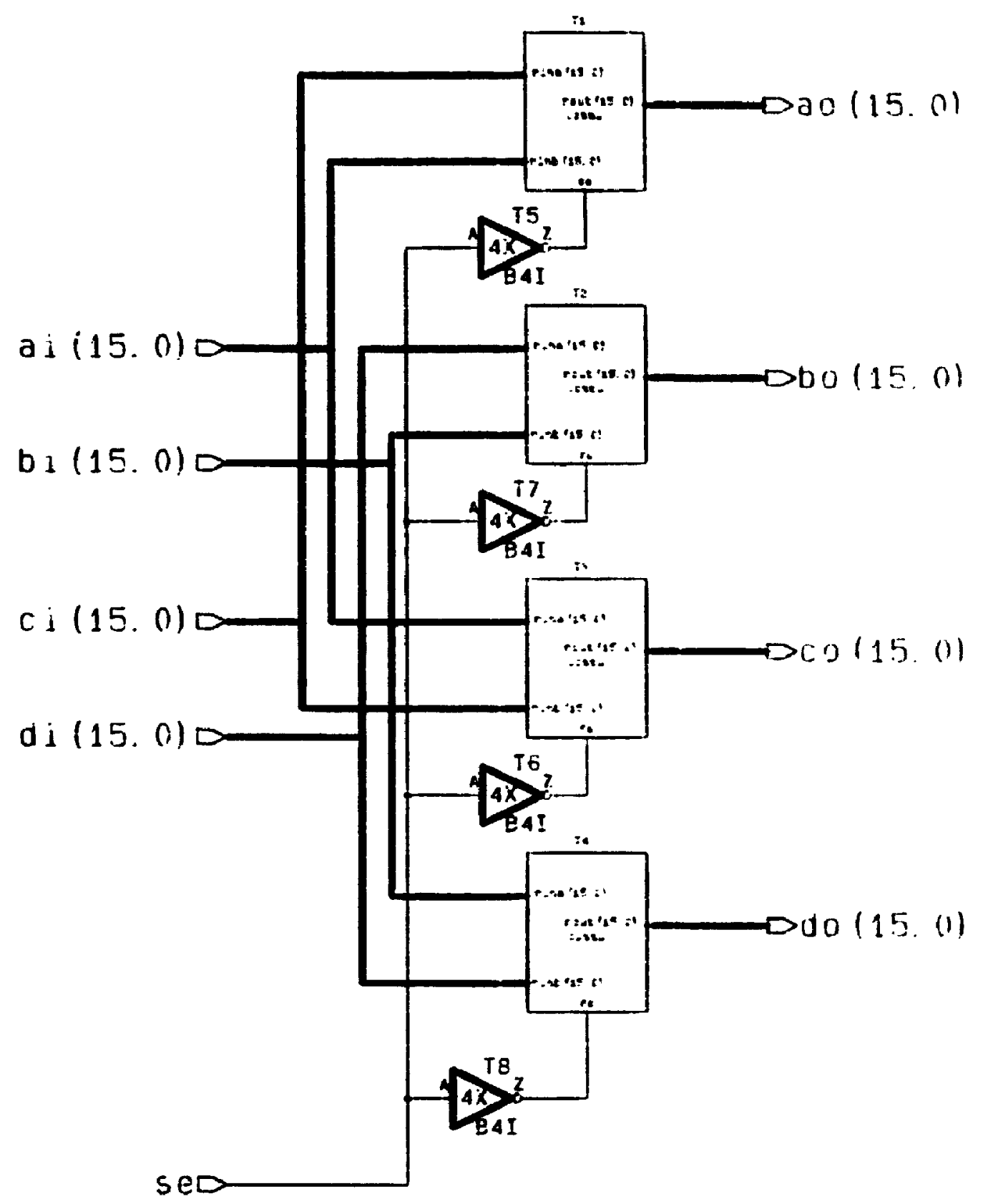

Figure 4.4 A Commutator with four sets of multiplexers 


\subsubsection{Control Unit Design}

\subsubsection{Introduction}

The Control Unit generates all the necessary control signals to ensure the correct operations of the Switch. External information required by the Switch is loaded onto the module only once prior to the start of the operation and the internal control signals are generated accordingly. Almost all control signals required by the Switch are generated within the Switch in order to minimize control overheads at the system level.

The Control Unit is divided into four parts which include: the clock cycles generator, synchronization pulse logic, cascadeability control and information latch. A description of each part of the Control Unit follows.

\subsubsection{The Clock Cycles Generator}

The function of the generator is to scale down the external master clock and provide the necessary clock cycles to all parts of the Switch. The initial specification of the master clock is $50 \mathrm{MHz}$ with a $50 \%$ duty cycle. This specification is used throughout the design and simulation processes of the Switch. Altogether, there are four clock prescalers within the generator that are responsible for the four internal clock cycles namely CP4a, CP4b, CP4c and CP4d. Each prescaler, shown in Figure 4.5, divides the master clock by four to provide a $12.5 \mathrm{MHz}$ clock cycle. As mentioned in section 4.3.1, each bit of delay line in $Z^{-k}$ block is partitioned into four sets of delay elements and these are clocked by the four separate CP4 cycles. Except for CP4a, the other three clock cycles are solely for clocking the inputs of the delay elements in the $\mathrm{Z}^{-k}$ blocks. Cp4a is responsible for the first set of registers, $\mathrm{CP} 4 \mathrm{~b}$ is responsible for the second set and so on. Each of the four clock cycles could be disabled to reduce the overall power 


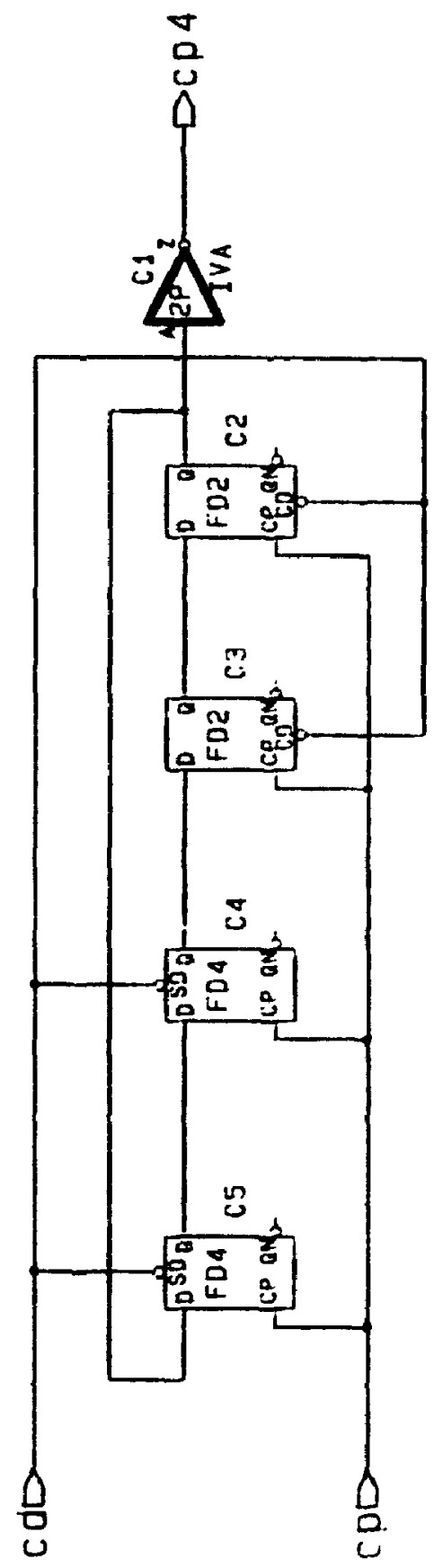

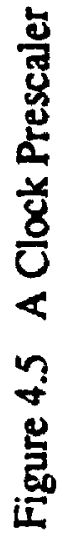


consumption of the FFT processor. For example, Figure 4.6 shows a block diagrain of a 32 point radix-2 pipelined FFT processor. The figure indicates that less than half of the delay elements are operating simultaneously and the rest are disabled. This example emphasizes that it is essential to be able to disable the unused delay elements in order to reduce the power consumption of the processor.

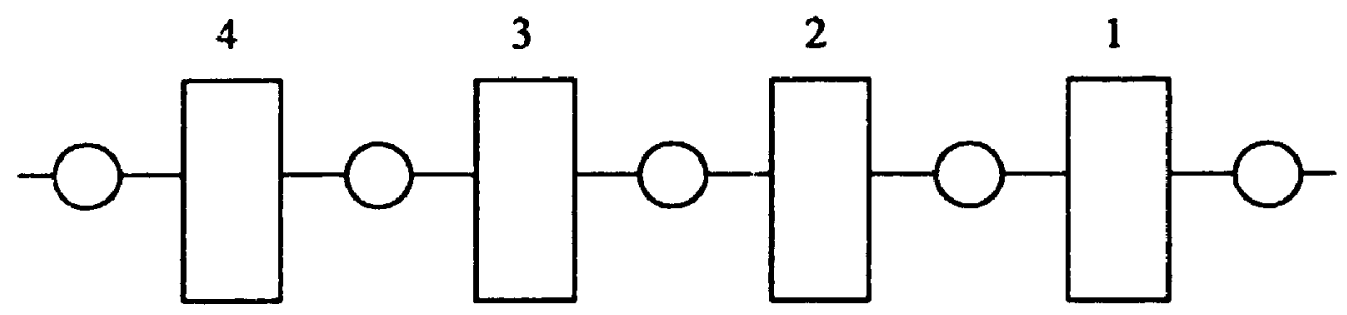

\begin{tabular}{|c|c|c|c|c|c|}
\hline Switch \# & 4 & 3 & 2 & 1 & Total \\
\hline $\begin{array}{l}\text { Delay } \\
\text { Elements in } \\
\text { operation }\end{array}$ & $64 * 8$ & $64 * 4$ & $64 * 2$ & $64 * 1$ & $64 * 15$ \\
\hline \multirow{3}{*}{$\begin{array}{l}\text { Delay } \\
\text { Elements } \\
\text { disabled }\end{array}$} & $64 * 0$ & $64 * 4$ & $64 * 6$ & $64 * 7$ & $\underline{64 * 17}$ \\
\hline & \multicolumn{5}{|c|}{ elements $=(\#$ of input bits $) *(\#$ of delay elements $)$} \\
\hline & & dtter & Swi & & \\
\hline
\end{tabular}

Figure 4.6 Delay elements of a 32 point pipelined FFT processor

\subsubsection{The Sync Pulse Logic (SPL)}

As mentioned in chapter three, the synchronization between data and reordering operations is ensured by using a Sync pulse which is generated at the system control of the FFT processor. The Sync pulse propagates through the Switch along with the data 
stream; however both "travel" in two independent channels. The reordering process is activated if the incoming data is valid and the valid data is identified by using a logic low signal on the Sync pulse. The negative going edge of the Sync pulse initiates the reordering process and the process continues indefinitely until the pulse returns to a logic high, thus terminating the process.

The function of the SPL is to provide exactly the same delays for the Sync pulse as for the data stream when it passes through the Switch. The delays in the SPL are also implemented by means of a shift register. Each switch mode processes the Sync pulse in a different fashion except the Output switch mode which handles the pulse similarly to the Right switch mode. Once the Principle Switch is identified in a set of cascaded Switches, additional delays are added onto the Sync pulse to compensate for the delays imposed by the Complex Butterfly to the data stream. This is necessary since the Sync pulse does not propagate through the Complex Butterfly. Sync pulse and data stream will be aligned such that both the start of the valid data and the negative edge of the Sync pulse will appear at the output of their respective channels of the Switch stage within the same clock cycle. The output of the Sync pulse (Syout) is directly fed into the Sync pulse input (Syin) of the following Switch even if the Butterfly is locilted between the Switches.

\subsubsection{The Cascadeability Control}

In order for the Switch to be cascadeable, it must be capable of operating in all five modes as explained in section 3.2.2. The FFT Switch responsible for the reordering of data is called the Principle Switch. It can be implemented as either the Middle or Endi Switch mode (as shown in Figure 3.2). Data reordering is accomplished by using a 
commutator with its select input controlled by the Reordering Frequency'. Since a Principle Switch is designed such that it can operate at any position in the pipeline, it must be capable of producing a spectrum of frequencies required for reordering. Figure 4.7 shows the reordering of the data through the Switches in a 32-point radix-2 pipelined FFT. It shows that the Reordering Frequency of the Principle Switch in stage 4 is half the frequency of the Switch in stage 3, which in turn is half the frequency of the Switch in stage 2, and so on. A binary up-counter with synchronized clear is chosen to generate the Reordering Frequencies. A twelve bit counter, with a tap at every stage, is used to generate all of the Reordering Frequencies required to support a single pipelined FFT processor of up to 8192 points. The twelve tapped frequencies are fed to a twelve-to-one multiplexer which is controlled by four Position signals. The Position signals are used to indicate the stage of the Switch within the pipeline. Subsequently, these signals will determine the correct Reordering Frequency for this particular stage.

The five Switch modes are selected by three sing!e bit Control signals. Once a Switch is assigned to a particular switch mode, it remains in the same mode until another set of Control signals is loaded.

\subsubsection{Information Latch}

The information latch block in the Control Unit is used to store and decode the Position and Control signals. Both Positien and Control signals are loaded through the information bus. For a 32-point pipelined FFT processor. all Switches and Butterflies on the same structure share the same bus. The Butterflies utilize the bus for the loading

1. The Rcordering Frequency is essentially a square wave which determines that the data either passes straight through or crisscrosses the commutator as shown in Figure 4.7. 
Input to the Switch in stage 4

Reordering

Frequencies

$$
\begin{aligned}
& 00010203040506070809101112131415 \\
& 16171819202122232425262728293031
\end{aligned}
$$

After first delay

00010203040506070809101112131415

16171819202122232425262728293031

Straight throwgh

Crisscross

Siraight threwgh

Data reordered and output of stage 400010203040506071617181920212223

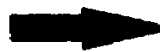
08091011121314152425262728293031

Stage 3 after the first delay 00010203040506071617181920212223

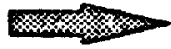
0809101112131415242526,27282430

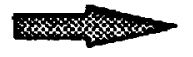

Output of stage 3

00010203080910111617181924252621 04050607121314152021222328243031

Stage 2 after the first delay 00010203080910111617181924252627 04050607121314152021222328293031

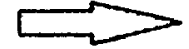

Output to stage 1 and after first delay

00010405080912131617202124252829 02030607101114151819222326273031

Output of the pipeline 凹ோ

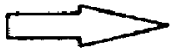

00020400081012141618202224262840 $01030507(691113151719212325272471$

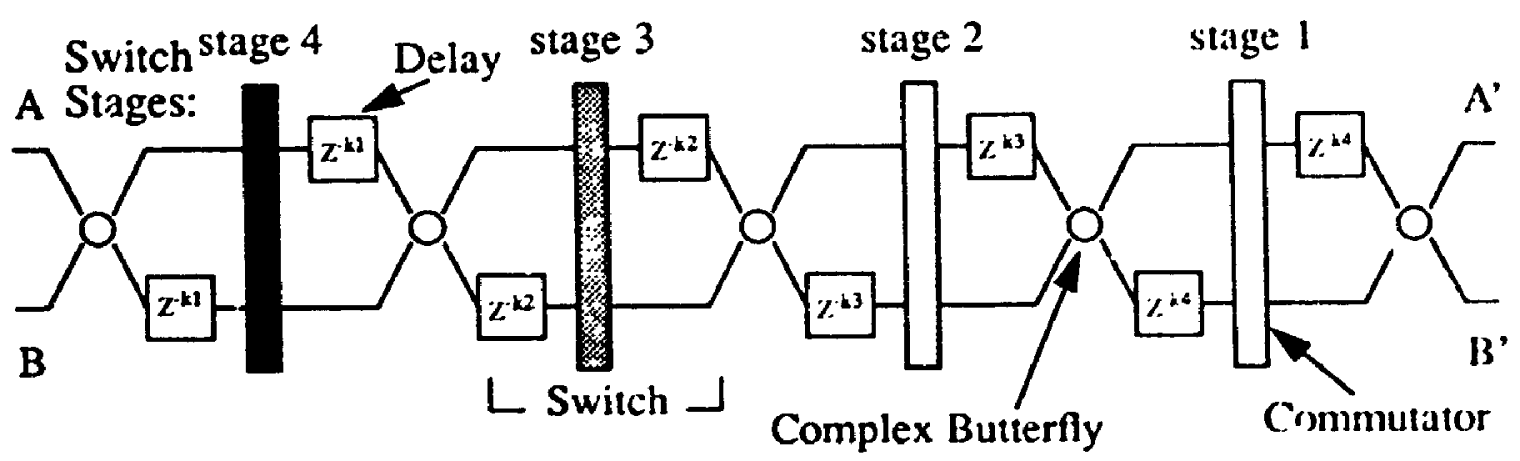

Figure 4.7 Operation of the 32 point pipelined FFT 
of twiddle factors. Information is sent through the bus to a Switch only once before the start. In order for the Switch to retain the loaded information, a series of latches, controlled by the Chip Select (CS) and Load Enable (SLOAD) inputs, is used. Information is loaded onto the Switch only when both CS and SLOAD inputs are at a logic low. Under this arrangement, SLOAD can be implemented as a broadcast signal for the FFT processor provided that a separate CS is implemented for each Switch and Butterfly. Once the information is latched, it is decoded and transferred to other parts of the Switch.

\subsubsection{Input and Output}

The input and output blocks are implemented such that data is clocked in and out of the Switch sequentially. Furthermore, a Linear Feedback Shift Register (LFSR) circuit is implemented in the input block such that it generates the pseudorandom test vectors necessary for self-test operation. The output block, it includes an LFSR based compacter circuit and predetermined signatures which are required to verify the results of the self-test.

\subsubsection{Design for Testability}

\subsubsection{Built-in Self-test}

With the rapidly increasing size of the VLSI designs, traditional methods of circuit testing become either impractical or very expensive. The built-in self-test (BIST) techniques are more popular than ever. They provide an efficient way of testing integrated circuits with large gate count and high pin out. Although testing is not required in both off-line and at-speed circumstances, the FFT Switch employs both 
techniques for implementing the BIST while remaining compatible with the Complex Butterfly on the system level.

The most popular techniques for built-in self-test involve providing a sequence of pseudorandom numbers on board as the stimulus and comparing the output of the circuit with the predetermined result (called a signature) at a fixed interval. One of the techniques involves using a read only memory (ROM) structure large enough to store a set of predetermined vectors and using them as input stimulus for testing. However, such an appreach is very time consuming since the effort necessary to determine the required set of test vectors to achieve the best fault coverage is substantial. Furthermore, a circuit with a large number of inputs will requires a large ROM structure to hold the necessary test set. By far the most popular method of generating test vectors in-circuit is to use an in-circuit pseudorandom sequence generator. The name pseudorandom reflects the fact that the output sequence is cyclic and not truly random. One such generator is called the Linear Feedback Shift Register (LFSR). An LFSR is a finite state machine consisting of interconnections of essentially two elements, namely memory element and modulo- 2 summer. The memory element and modulo-2 summer can be implemented as a shift register and exclusive-or gate respectively. The circuit overhead to produce test vector for a circuit with a large number of inputs is considerably less for LFSR than when using an ROM structure. The maximum period for an n-stage LFSR is $2^{\mathrm{n}}-1$ clock cycles (no all zero vector) and the generating function corresponding to a maximum period LFSR is called a primitive polynomial.

For the FFT Switch, a sixteen bit LFSR, shown in Figure 4.8, is employed to generate two primitive polynomial pseudorandom sequences. It is located at the input 
block of the Switch. The two polynomials shown in Equations (4.1) and (4.2) are chosen to generate the pseudorandom sequences.

$$
\begin{aligned}
& p=x^{16}+x^{14}+x^{13}+x^{11}+1 \\
& p^{\prime}=x^{16}+x^{5}+x^{3}+x^{2}+1
\end{aligned}
$$

These two polynomials are published in the literature [28][29] along with a number of other polynomials for different bit sizes. Although both polynomials are primitive, the pseudorandom sequence of the Equation (4.1) is in reverse order of the Equation (4.2).

A sixteen bit Multiple Input Pseudorandom Compacter (MIPC) along with two signatures is located at the output block of the Switch. The MIPC is based on an LFSR architecture with an additional exclusive-or gate at every stage. The MIPC structure is shown in Figure 4.9. The compacted vector is clocked sequentially into the signature analyser and compared with the predetermined signatures. A single output pin called GO_NOGO indicates the status of the circuit. The GO_NOGO output is at the logic high level at the beginning of the self-test procedure and it will toggle to a logic low for a CP4 clock cycle (80ns) when a compacted vector matches the signature. Since there are two pseudorandom sequences, two signatures are chosen in each test cycle with one located at the middle and the other at the end of each cycle. When the compacted vector from the first sequence matches the first signature located at the middle of the test cycle, all registers in the $S$ witch are reset and ready for the second sequence generated from the same LFSR structure. Once the second signature is matched, all registers will be reset again and the self-test procedure will repeat itself by using the first sequence. Hence, if the Switch is held in the self-test mode, a periudic waveform will be present at the GO_NOGO output provided that the module passes the self-test routine. The 


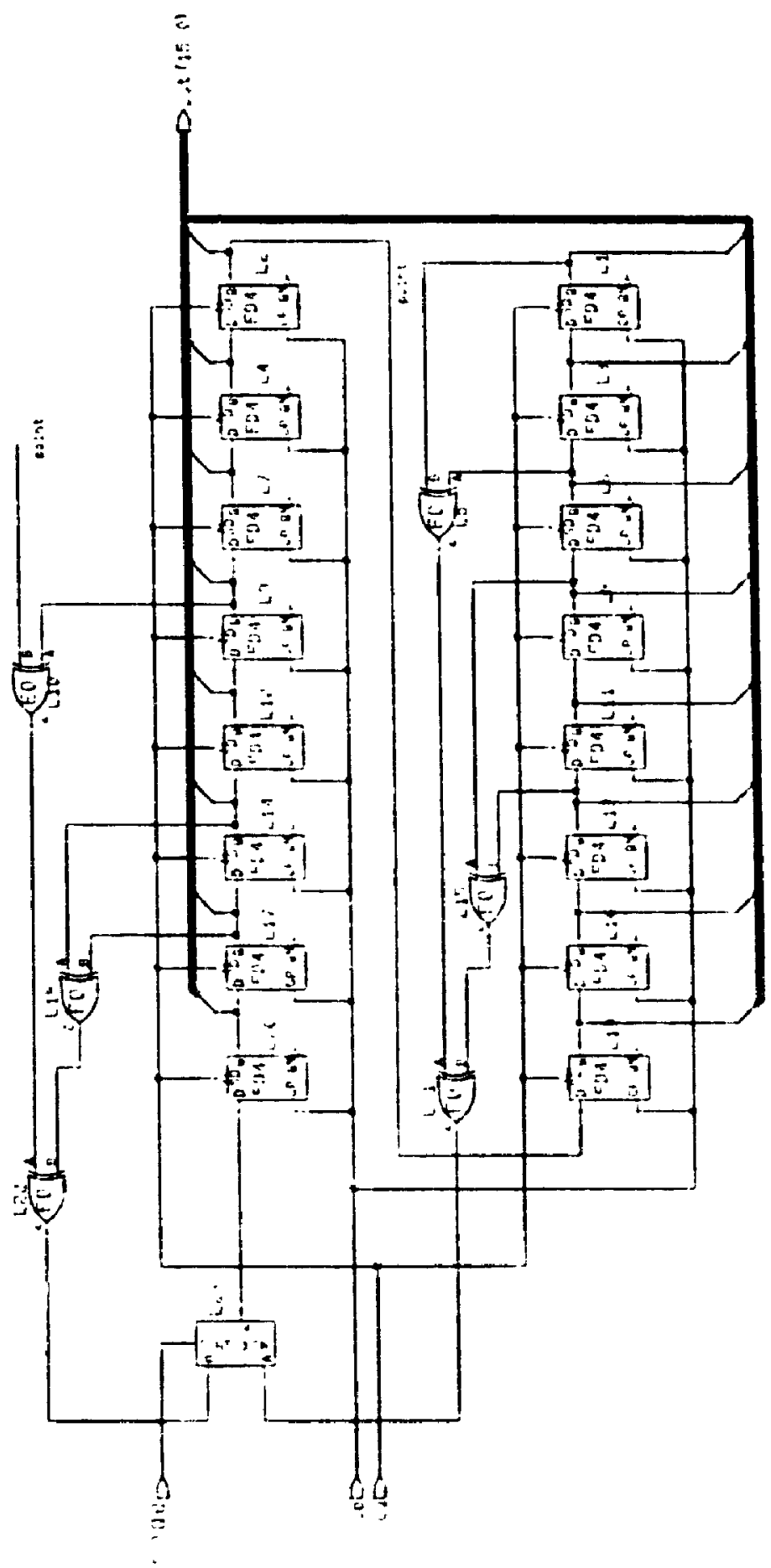

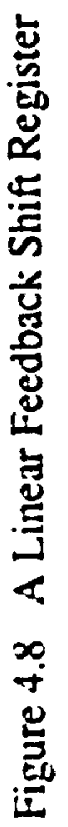




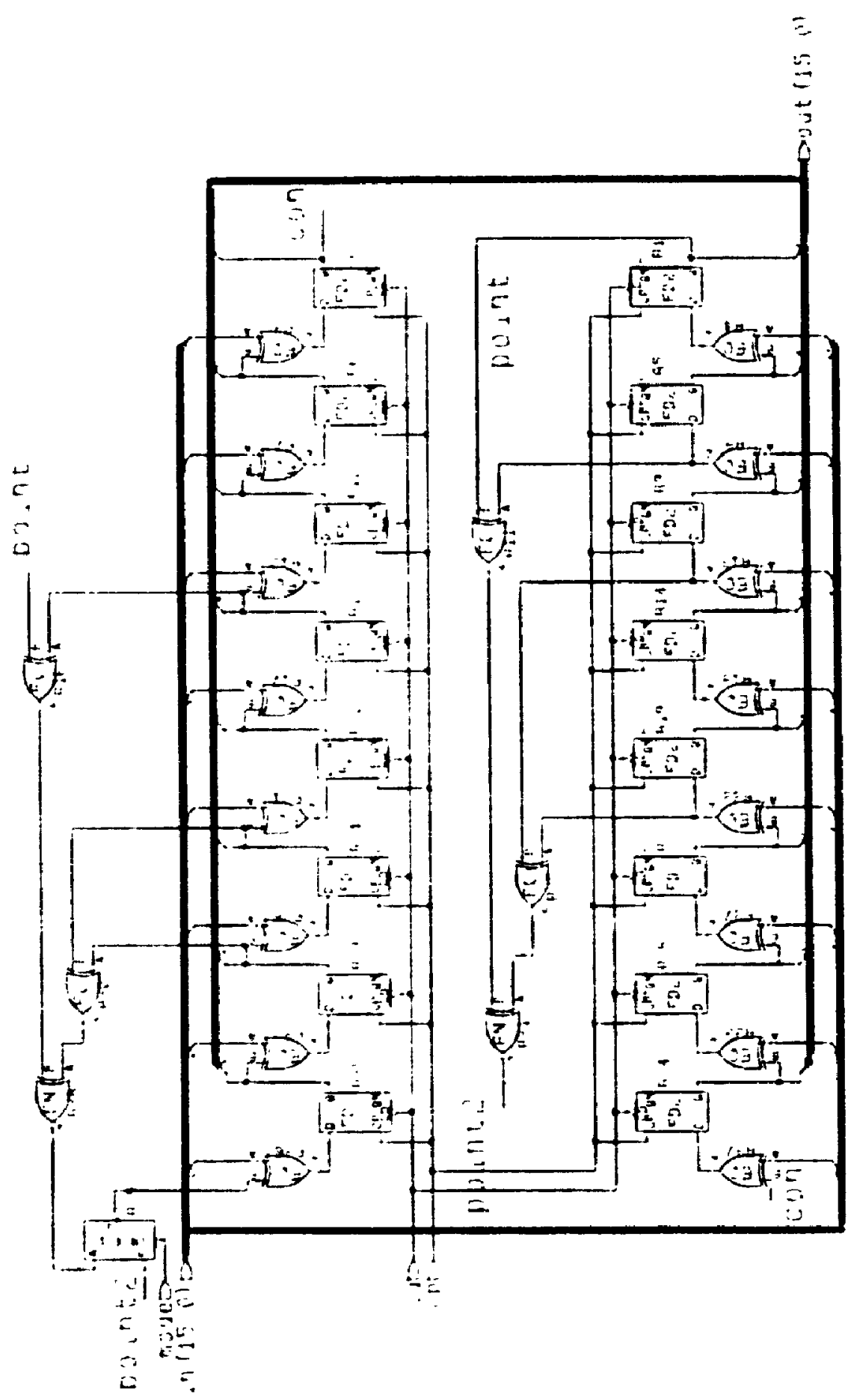

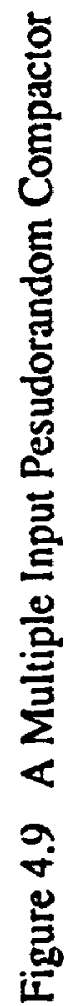


operation of a two-signature scheme reduces the frequency of aliasing from $1 / 2^{\text {n }}$ for a single signature to $1 / 2^{2 n}$ for two signatures $[30$, thus increasing fault coverage. Aliasing occurs when the circuit under test produces the same compacted vectors even when it contains faults. The additional hardware cost to implement such a scheme is small, and it has the additional benefit of doubling the test length for pseudo-cxhatustive testing. However, due to the memory constraint ${ }^{1}$ of the available IC tester, it has nut been possible to apply the full length of the pseudorandom sequence to the FIFI Switch. Instead, the LFSR will produce a sequence for each polynomial of just over $2(x)(x)$ lock cycles.

\subsubsection{Interconnectivity Test}

Apart from the implementation of LFSR and MIPC as a self-test techuicjue ws cover the core of the Switch, additional circuitry is added for both the Switch and the Butterfly to test the $\mathrm{I} / \mathrm{O}$ buffers and more importantly, the interconnectivity of cascaded modules. The method is based on the usage of a parametric NAND tree structure present at the input buffer of every ASIC. The NAND tree structure is required by the foundry for the input threshold voltage measurements. Figure 4.10 shows the input buffer incorporated with a NAND gate for the parametric NAND tree $^{2}$. $\Lambda l l$ input pins are held at logic high at the beginning of the test. Then the inputs are toggled to logic low sequentially. The output of the NAND tree is sent to the TESTOUT pin and it is expected to toggle continuously if there are no disconnections between the modules. It is possible to test the connectivity of a series of ASICs by connecting the NAND trees in series and testing each ASIC in sequence. Additional multiplexers are implemented

1. The I.C. rester is able to store a set of simulated results of up to 4000 clock cycles.

2. A more detailed explanation of the parametric NAND uree can be found in Appendix $C$. 


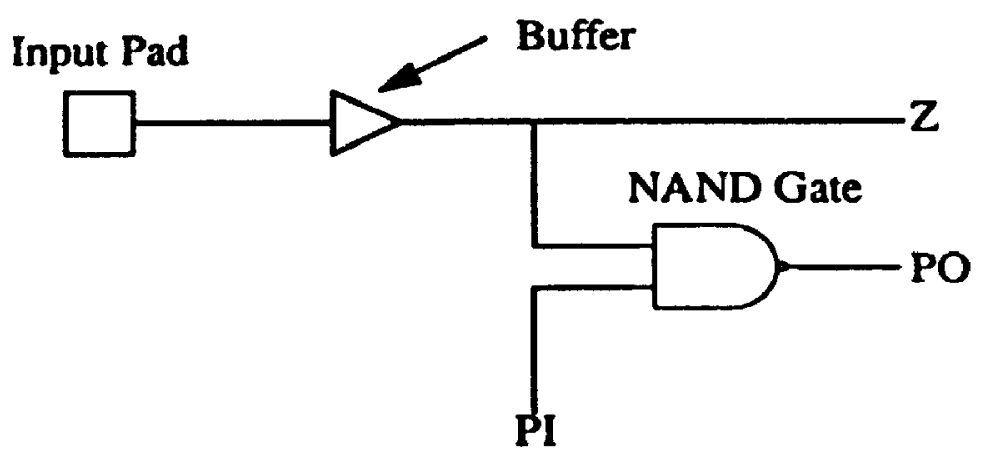

PI : Parametric tree input from the NAND gate output of the previous stage PO : Parametric tree output to the following stage

Figure 4.10 An input buffer with a NAND gate for a parametric NAND tree.

at the output of each module so that any TESTOUT signal is able to bypass a number of chip modules providing the required controllability and observability. The test commences with the first module on the pipelined structure, then the second module. and so on down the pipeline.

\subsection{Summary}

In this chapter, a circuit description of the FFT Switch, divided into separate sections based upon the functionality of each block has been presented. The design of each functional block was carried out so that the technological, performance and system constraints of the FFT Switch were reflected. Simulation results to verify the implementation of the FFT Switch will be given in the next chapter. 


\section{Chapter Five}

\section{Simulation and Test Results}

\subsection{Introduction}

In this chapter, simulations and test results of the FFT Switch are presented io verify the functionality of the module. The Switch is simulated either as a stand-alone module or a two-module cascaded structure. The stand-alone module represents just one Switch in the simulation and the two-module cascaded structure represents thre: different combinations, namely "Switch-Switch", "Switch-Butterfly" and "ButterflySwitch" where all combinations include at least one FFT Switch. All simulations are performed under the worst case, the best case and the nominal case commercial conditions. The worst case commercial condition simulations are done under conditions where the power supply is assumed to be $4.5 \mathrm{~V}$ and the ambient temperature $70^{\circ} \mathrm{C}$; the best case commercial condition assumes a $5.25 \mathrm{~V}$ power supply and $0^{\circ} \mathrm{C}$ ambient temperature; and the norminal case corresponds to $5.0 \mathrm{~V}$ and $20^{\circ} \mathrm{C}$. 


\subsection{Simulations of the FFT Switch}

\subsubsection{Stand-alone module}

\subsubsection{Introduction}

The simulations of the FFT Switch are divided into two major sections, namely functional and non-functional verifications. For functional simulations, emphasis will be placed on the various switch modes and different number of delays by changing the combinations of Positional and Control signals. Further simulations are also done in the self-test mode. As for non-functional simulations, these include fault simulation, toggle coverage simulation and post layout verifications. The post layout verifications are required by the foundry and will be explained in a later section.

\subsubsection{Functional Test}

As mentioned in Chapter 3 and 4, in order for the Switch to operate as a generic building block for a pipelined FFT processor, architecturally it must be capable of implementing five different switch modes, namely the Middle End, Right, Left and Output switch modes. Besides the Middle switch mode, Switches which operate in the other four modes do not function as a single module in an FFT processor and they are required to cascade with other Switches during normal operations in order to provide the necessary number of delays between Complex Butterflies. Nevertheless, to verify the functionality of the different switch modes, all five modes are individually simulated and the data stream and Sync pulse outputs are compared with the predicted results. 
Example 1: The following two simulations correspond to Switch $A$ and Switch D of a 32 point FFT processor (shown in Figure 5.1) where Switches $A$ and D represent the last and the first Switch of the pipeline respectively. All four switches operate in the Middle switch mode.

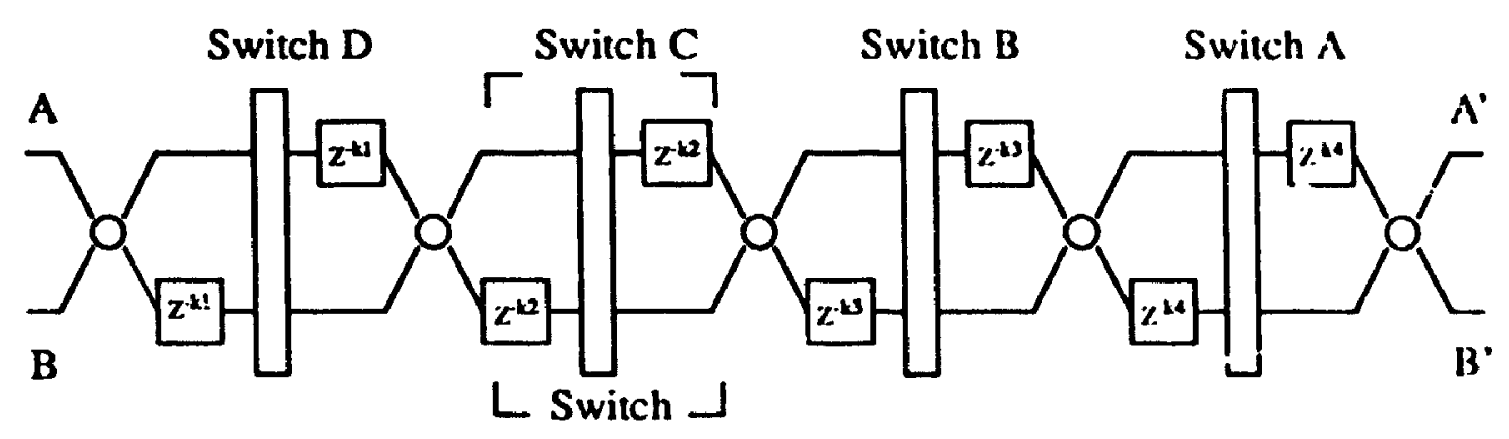

Figure 5.1 A 32 point pipelined FFT processor

Figure 5.2 shows the simulation results of Switch $A$. The input stimulus is selected so that results at the output can be easily interpreted. Besides the two clock cycles delay due to the input and output blocks, the simulation shows that the $Z^{-k}$ blocks impose only one clock cycle delay on each data stream, hence the location of the Switch can be verified as the last FFT Switch in the pipelined FFT processor. The Reordering Frequency, which controls the select input of the middle commutator in the Switch, is identified as the signal called "/A91/Z6/swi2" in Figure 5.2. The Reordering Frequency shown in the plot represents the highest frequency that can be provided by the FF: Switch. Depending upon the location of the FFT Switch relative to the pipeline, the Reordering Frequency may alter. This is required to ensure that the data reorders correctly throughout the FFT processor. As the simulation resulis have indicated, apart from the clock pulse CP4a, the other three internal clock pulses (CP4b, CP4c and CP4d) 


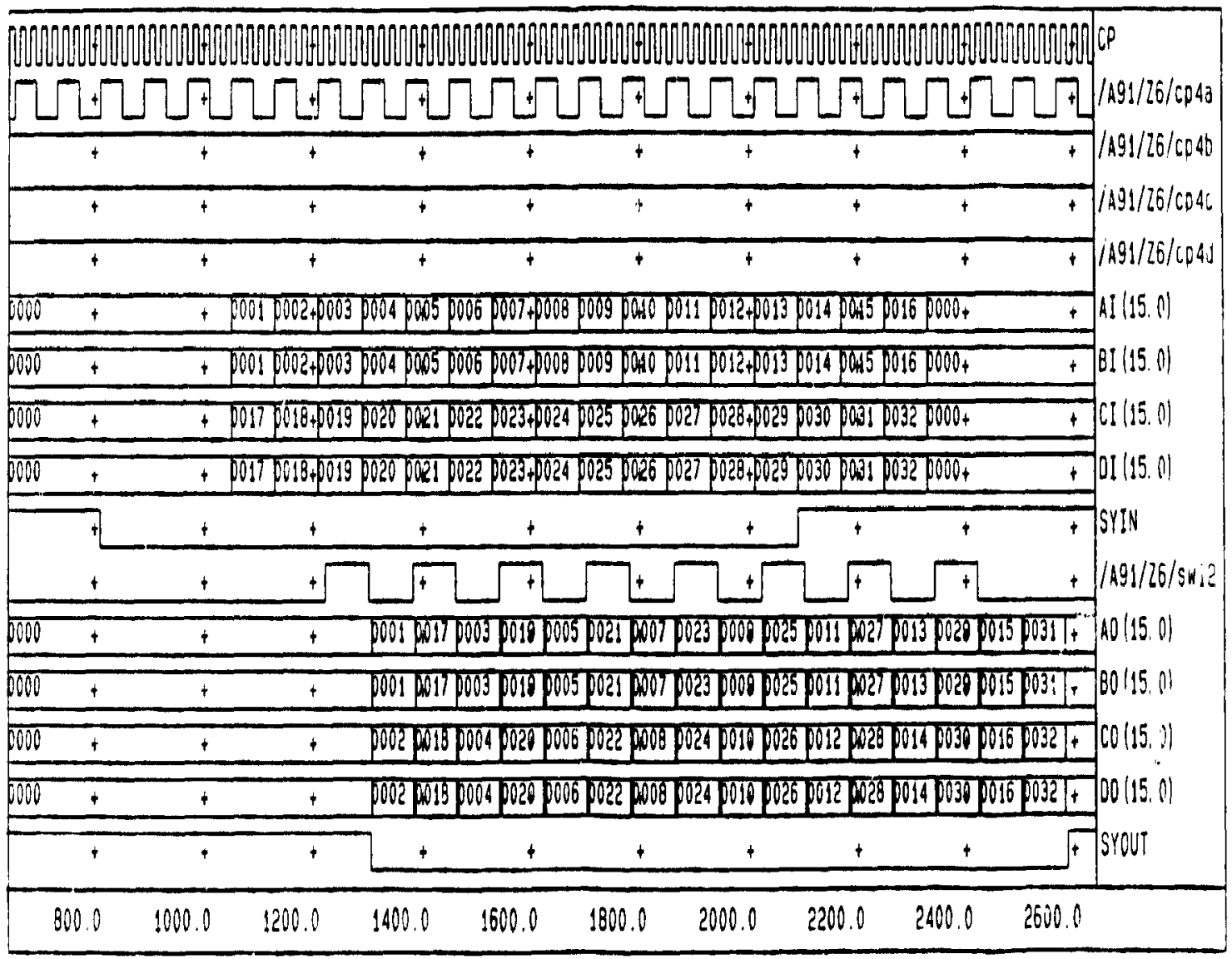

Frgure 5.2 Smulation result of iwatih A in Example 1 
are shown to be disabled. Since the simulation represents the last FFI Switch, only the first set of shift registers in the $\mathrm{Z}^{-\mathrm{k}}$ blocks are utilized. The remaining registers are disabled in order to reduce the power consumption of the Switch. Initially, the valid datti at the input is three CP4 clock cycles behind the Sync pulse. These three clock cycles are imposed on the data by the Complex Butterfly which is located just before the Switch $\Lambda$. As the simulation shows, extra delays are placed on the Sync pulse such that the data strean and the pulse are once again aligned at the output.

Figure 5.3 shows the simulation of Switch D which also operates in the Middle switch mode. The Positional signals of the Switch for this simulation have been altered in order to change the delay in the $Z^{-k}$ blocks and the Reorder Frequency for a different location on the pipeline. The number of delayed clock cycles imposed on the datat stream shown in Jigure 5.3 is increased to eight clock cycles. This is the maximum number of delayed clock cycles provided by a single $\mathrm{Z}^{-\mathrm{k}}$ blocks hence there is no clock pulse being disabled since both $\mathrm{Z}^{-k}$ blocks are fully utilized. The Reordering Frequency of Figure 5.3 is reduced eight-fold when compared with the same signal in Figure 5.2. Results for Switches $B$ and $C$ can be found in Appendix $A$ in order to complete all simulations of FFT Switches in a 32 point pipelined FFT processor.

Example 2: Apart from the Middle switch mode, the module operating in the End switch mode is also considered a Principle Switch which is responsible for data reordering. An eximple of a Switch which operates in the End switch mode is shown in Iigure 5.4. The two-Switch cascaded structure includes an End switch mode and an Output switch mode. In Figure $5.5^{\prime}$, the simulation result of the End switch mode shows

1. The simulation diagram is drawn accordirg to the actual simulated result. 


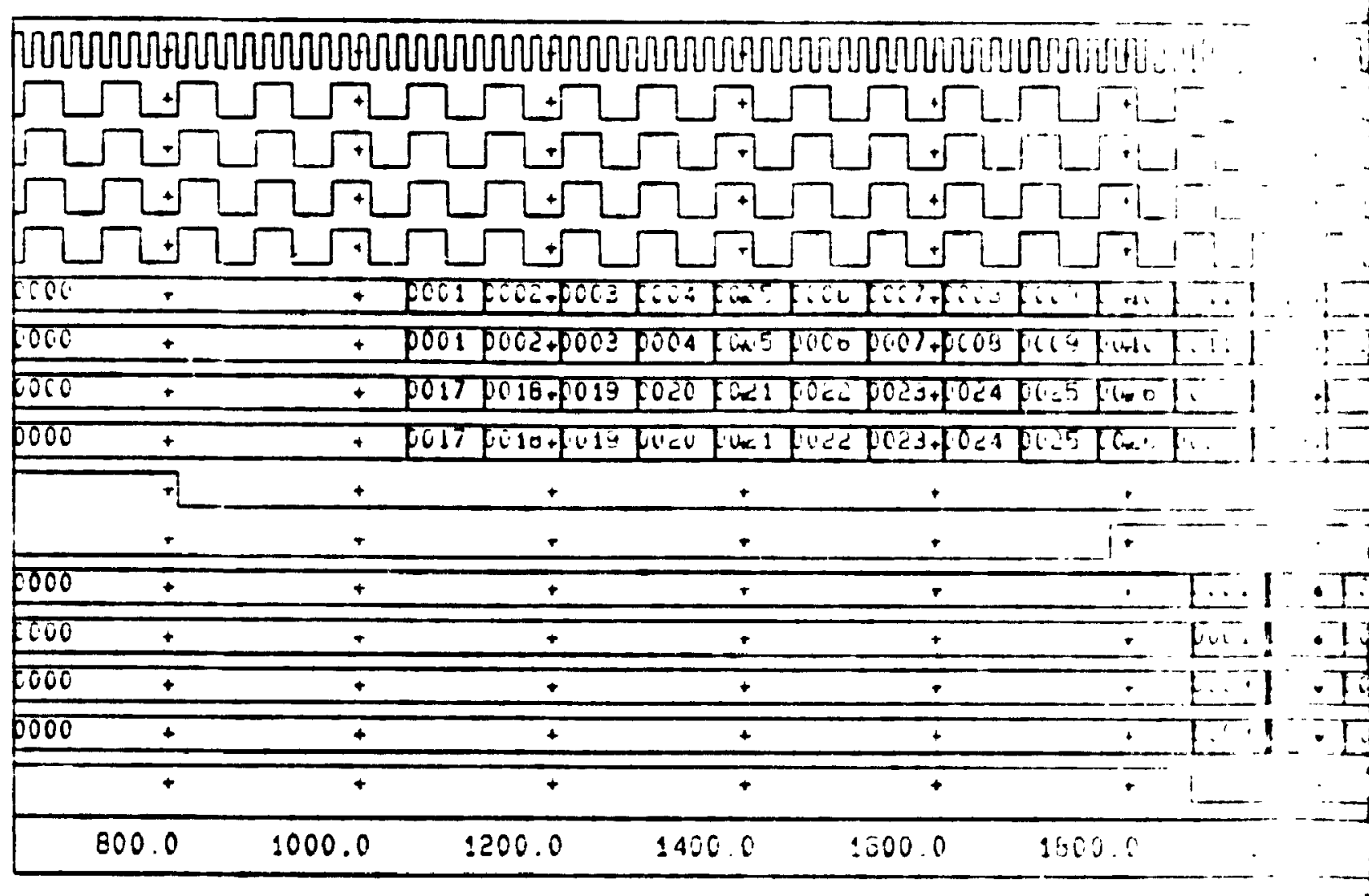

Fagures.z ramblat.at, 


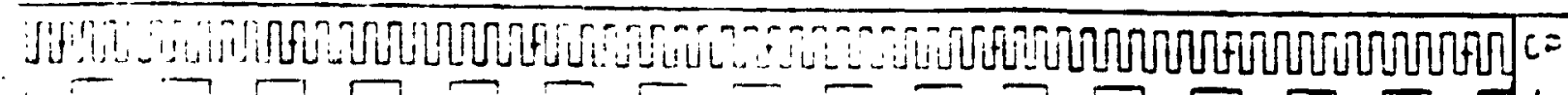

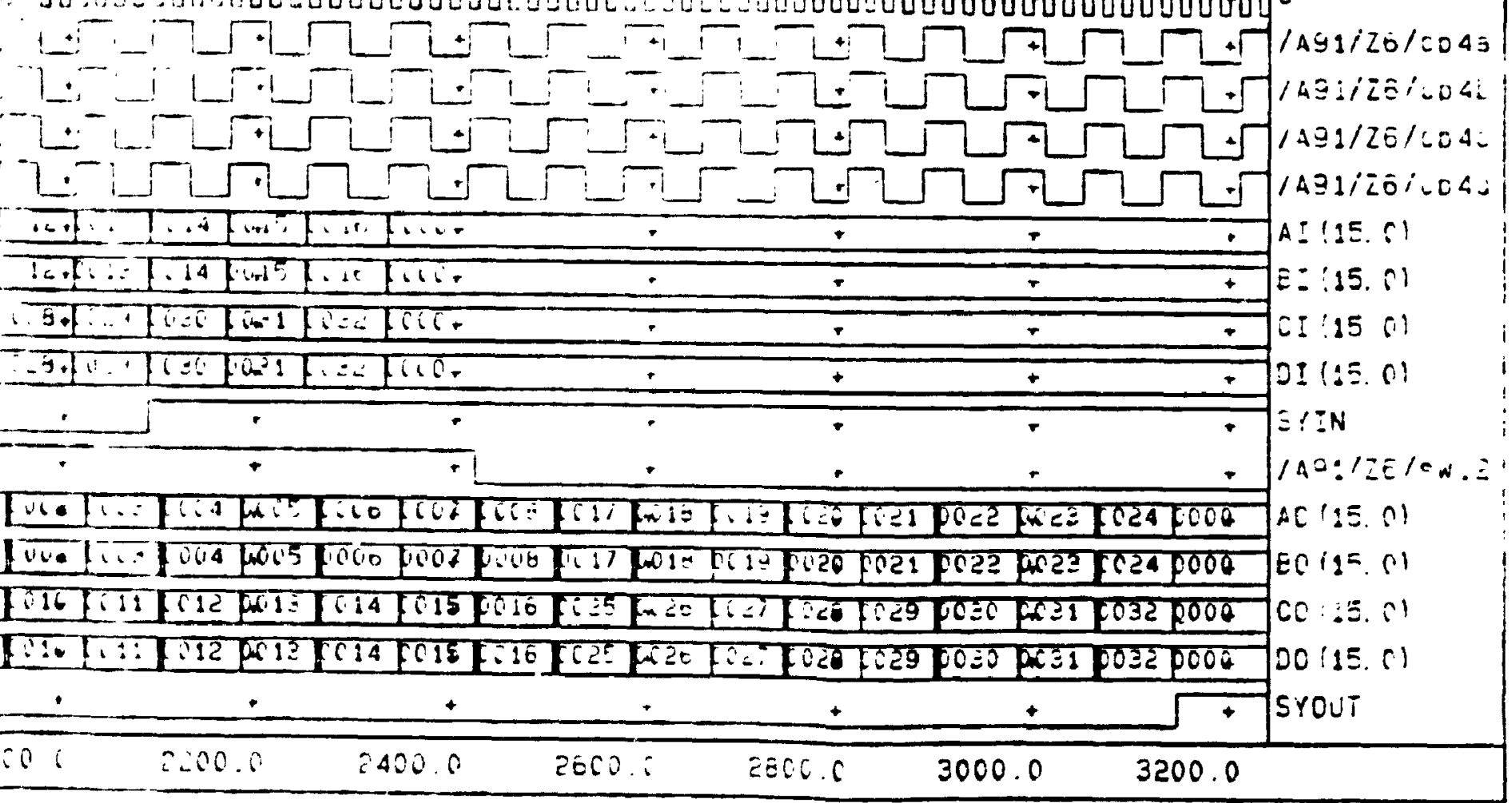

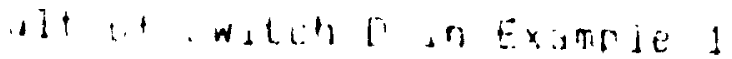




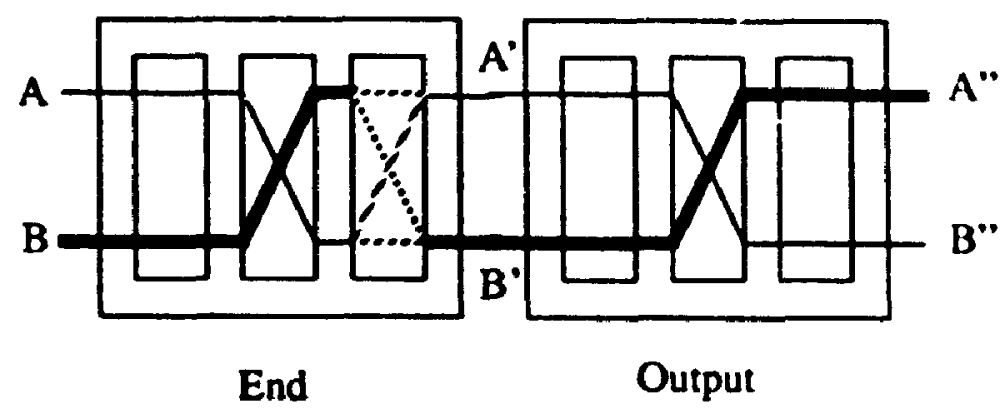

Figure 5.4 A two Switches cascaded structure

the input data on channel $B$ which is delayed and has crisscrossed to output $A^{\prime}$. No delay is applied on input data on channel A, however, part of the data will be crisscrossed to output $B^{\prime}$. In order to direct the overall output of the cascaded structure so that there is no data misalignment and misplacement at the outputs A" and B", a second FFT Switch is used. The function of the second Switch is to apply the correct amount of delay on the data from output B' so that both outputs $A$ " and B" will be aligned once again. Figure 5.6 shows the simulation of the second Switch and the Sync pulse defines the valid data at the output of the cascaded structure.

Example 3: Another example of a Switch which operates in the End switch mode is shown in Figure 3.4 and again in Figure 5.7. As the data passes through the inputs of the cascaded Switches, the data on channel B will be delayed by sixteen clock cycles at the output of the first Switch which operates in Left switch mode. An additional sixteen clock cycles delay is imposed on channel B by the second Switch. Figure 5.8 shows the simulation results at the output of the second Switch which is operated in the End Switch mode. The negative going edge of the Sync pulse is aligned 


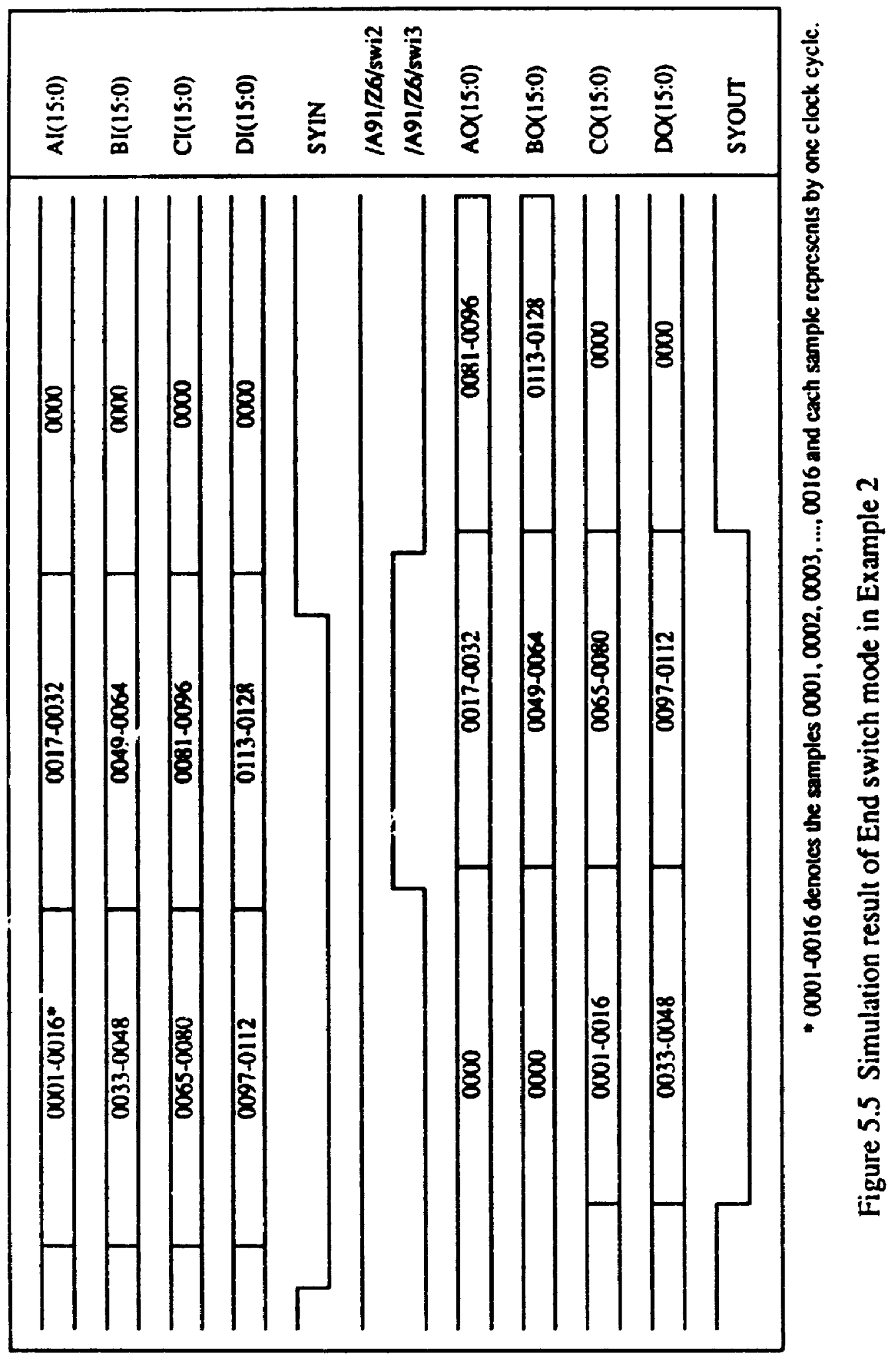




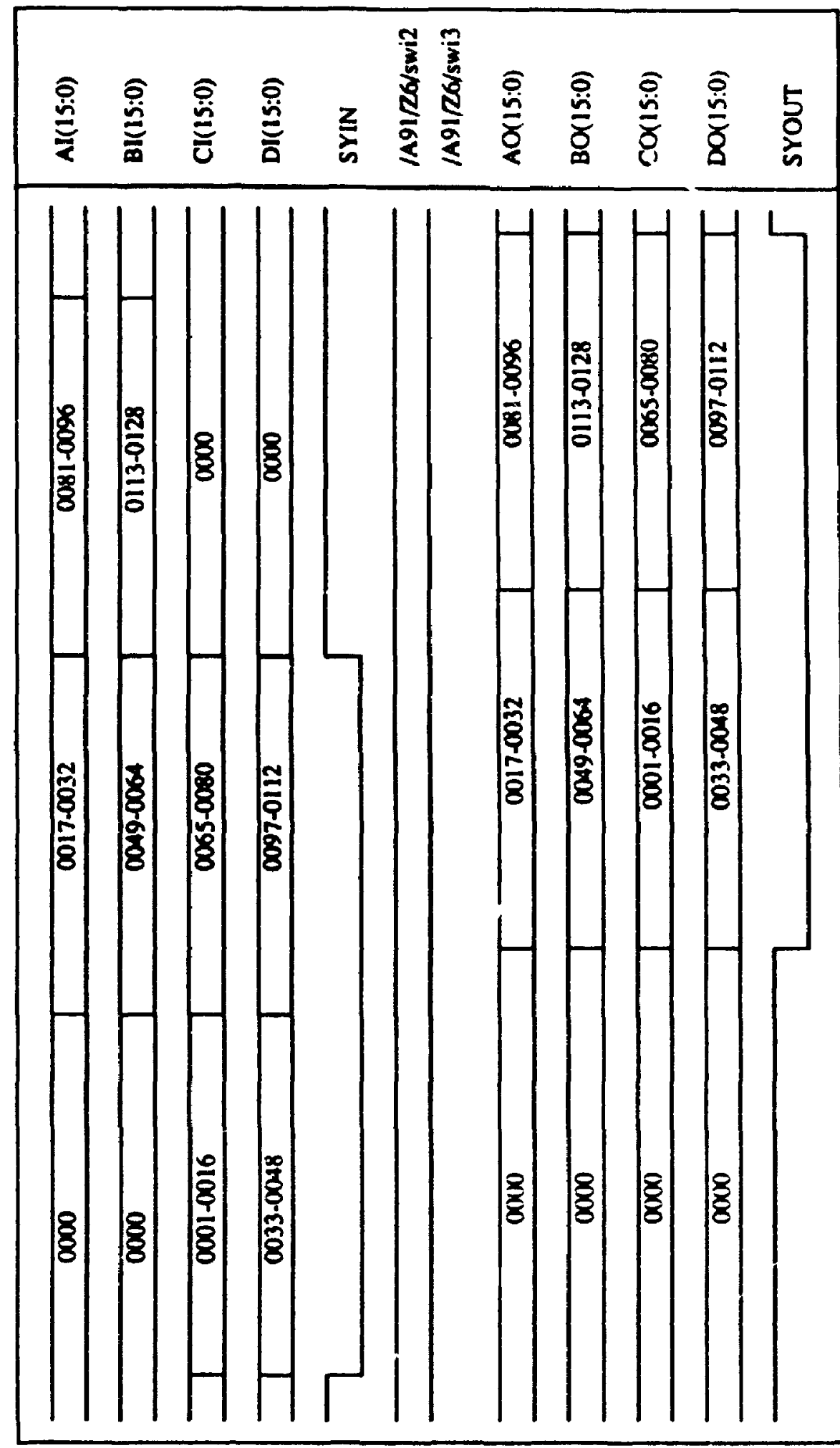

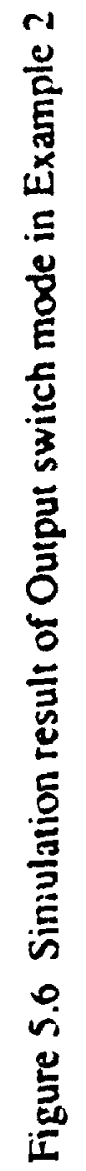




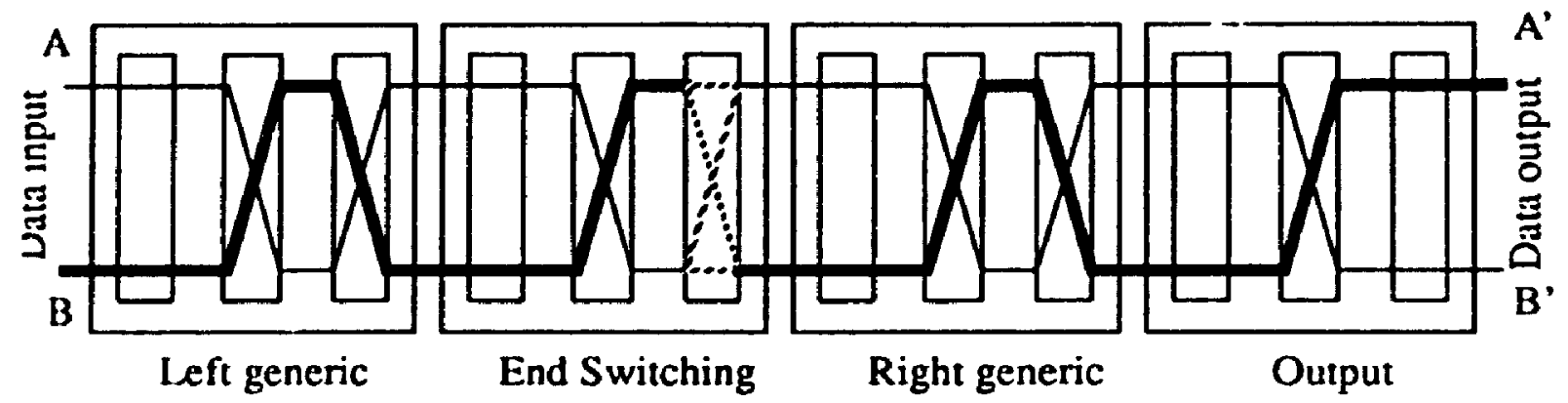

Figure 5.7 A four Switches cascaded structure

with the start of valid data of channel $A$. The simulation results show no clock pulse in the Switch which is disabled since both $Z^{-k}$ blocks are fully utilized. The simulations for Left. Right and Output switch modes for the same cascaded structure can be found in Appendix B.

Combining the simulations of the previous examples, including the four Middle switch mode modules, the two-Switch cascaded structure and the four-Switch cascaded structure. operations for Switches in all six stages of a 128 point pipelined FFT processor are demonstrated.

Example 4: Whereas both the Middle and the End switch modes are handled as Principle Switches, Switches which operate in the other three modes are used solely for routing and to provide extra clock delays for data before and after the reordering process. The difference between the operations of the Left and Right switch mode can be demonstrated by using the same data input and Sync pulse in both simulations. 


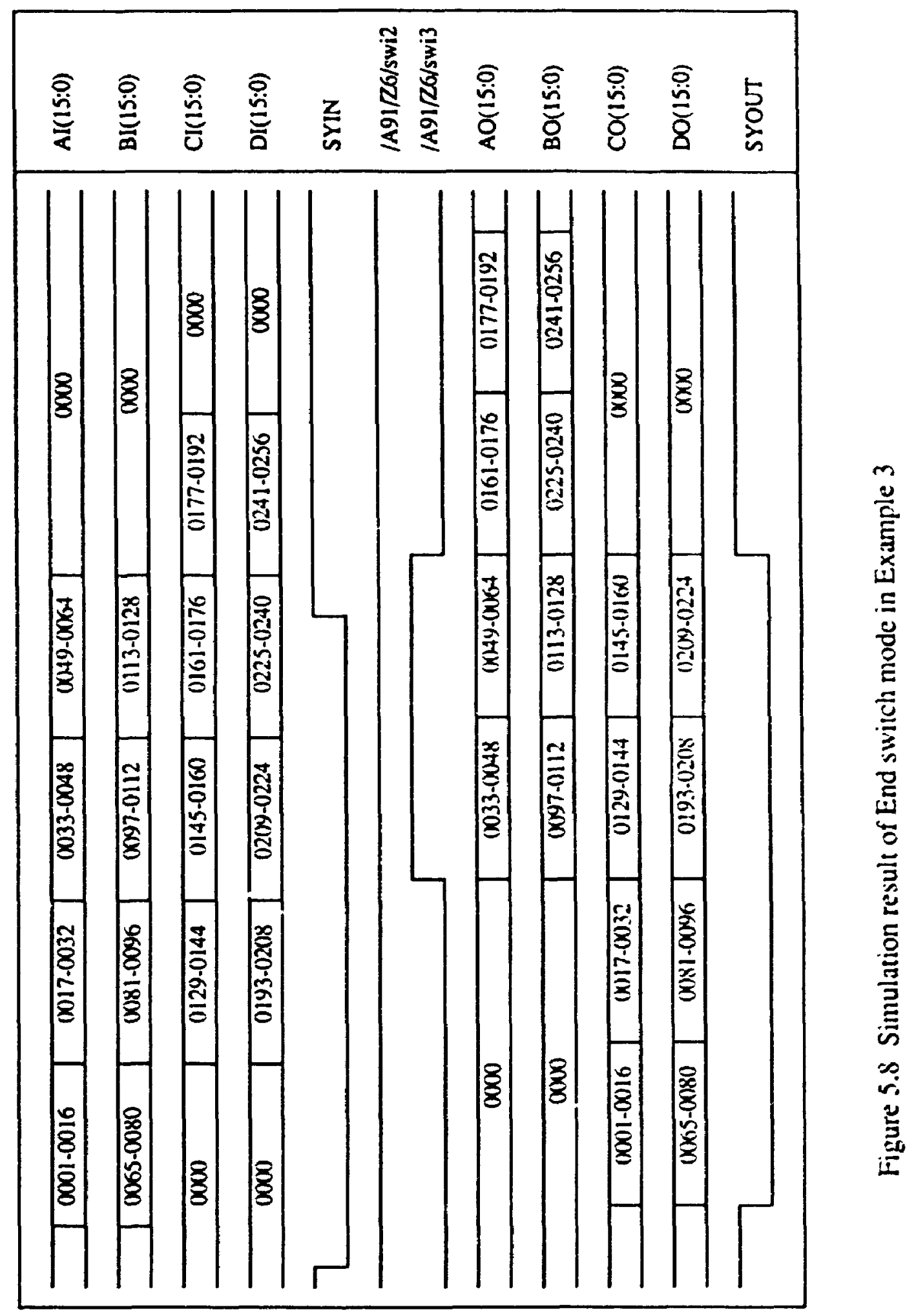


Figure 5.9 and Figure 5.10 show the simulations or Left and Right switch modes respectively. The only major difference between the operations of the two switch modes is the response of the Sync pulse. It is shown in in Figure 5.10 that the Sync pulse is delayed by sixteen clock cycles. This is necessary to ensure the Sync pulse is aligned with the start of valid data in the pipeline. The same input data as the Left and Right Switch modes is used to simulate a Switch which operates in the Output mode in order to distinguish the differences between these modes. Simulation results of the Output switch mode in Figure 5.11 shows that in comparison to the previous two switch modes. the data outputs of the two channels are crisscrossed. However, the response of the Sync pulse is the same as that of the Right switch mode.

Example 5: Whereas the five switch modes mentioned above are supposed to operate under on-line processes, the self-test mode is an off-line operation. Figure 5.12 shows the simulated response of Li: GO_NOGO output when the Switch is operating in the self-test mode. The response of the GO_NOGO oitput is a periodic waveform and along with the $80 \mathrm{~ns}$ logic low pulses, it indicates that the self-test is operating successfuliy.

\subsubsection{Non-functional Simulations}

Non-functional simulations of the FFT Switch include a fault simulation, a toggle coverage test and foundry-required post layout verification tests. The fault simulation is done by using a fault simulator called QUICKFAULT. A stuck-at fault coverage of $84 \%$ is recorded using the self-test procedure. As for the toggle coverage, $99 \%$ coverage is achieved by using the self-test procedures and a functional test routine. Additional simulations are required by the foundry for three other verifications. These 


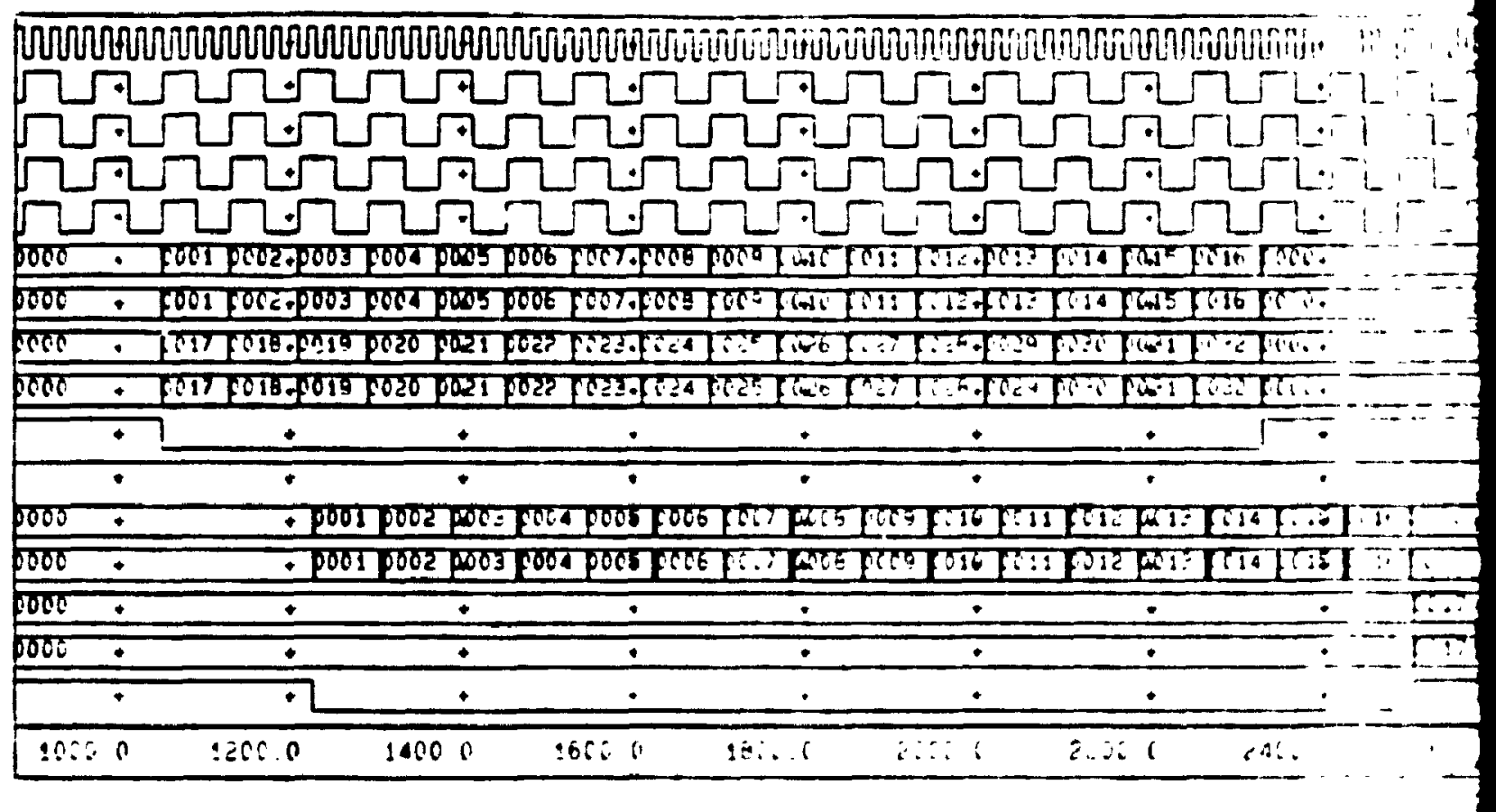




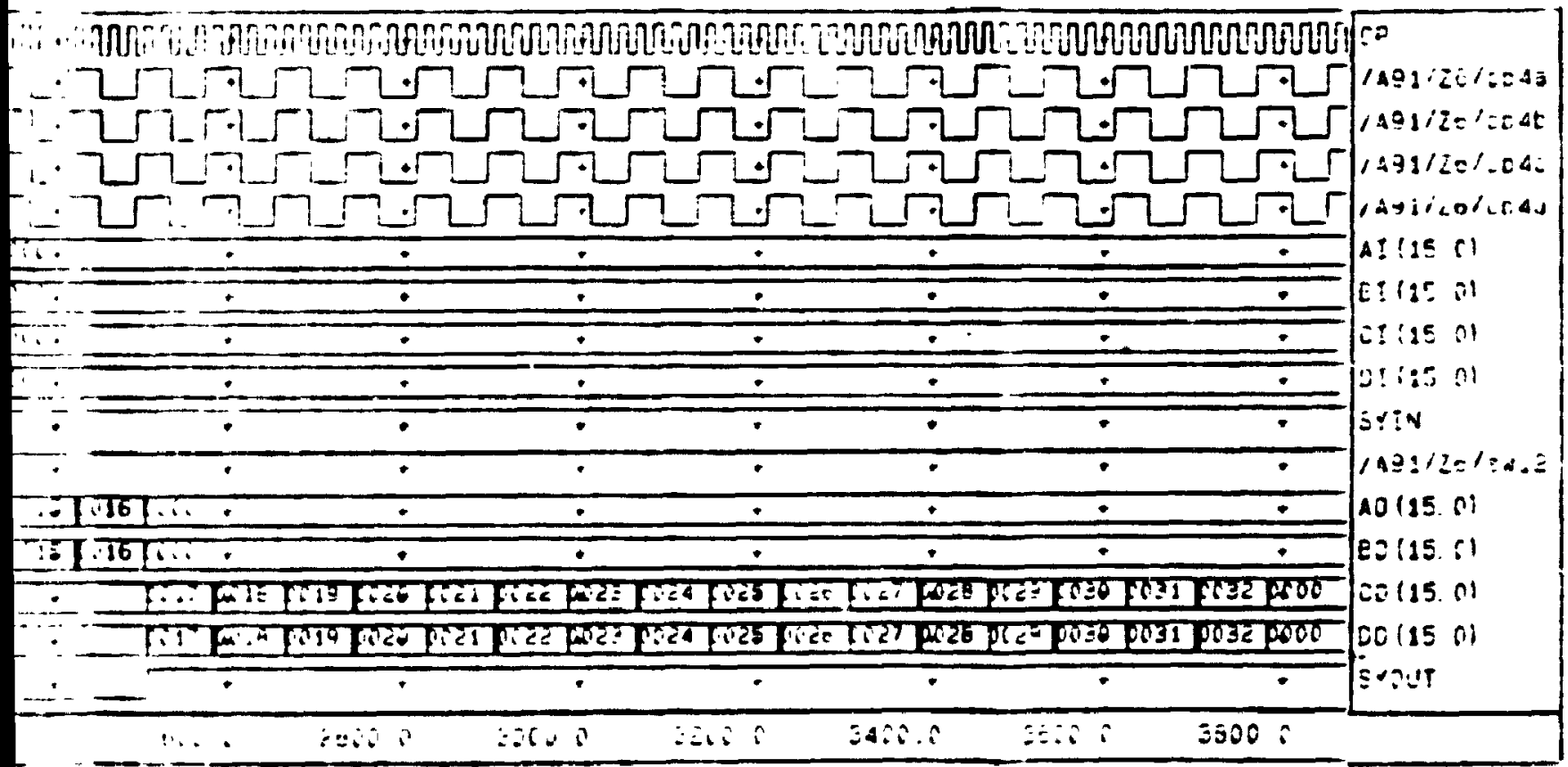




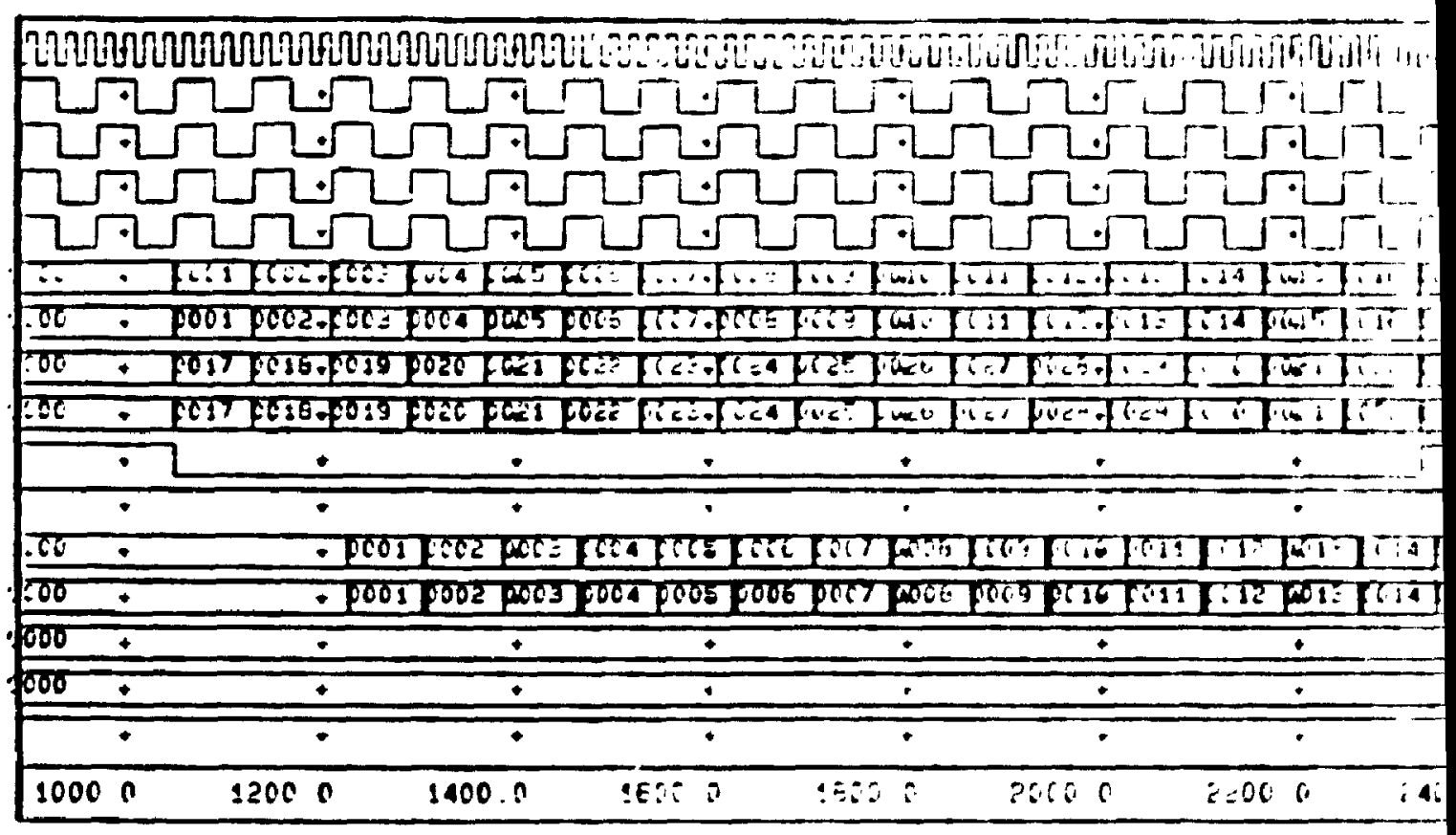

Fjoure 5.j0 camilat 


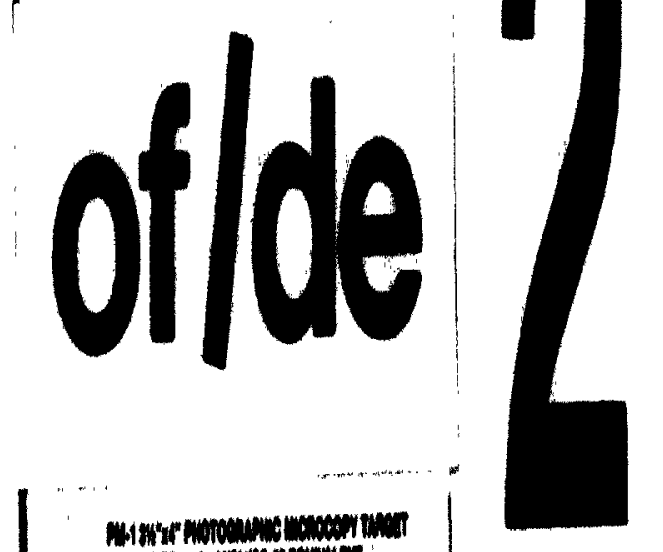

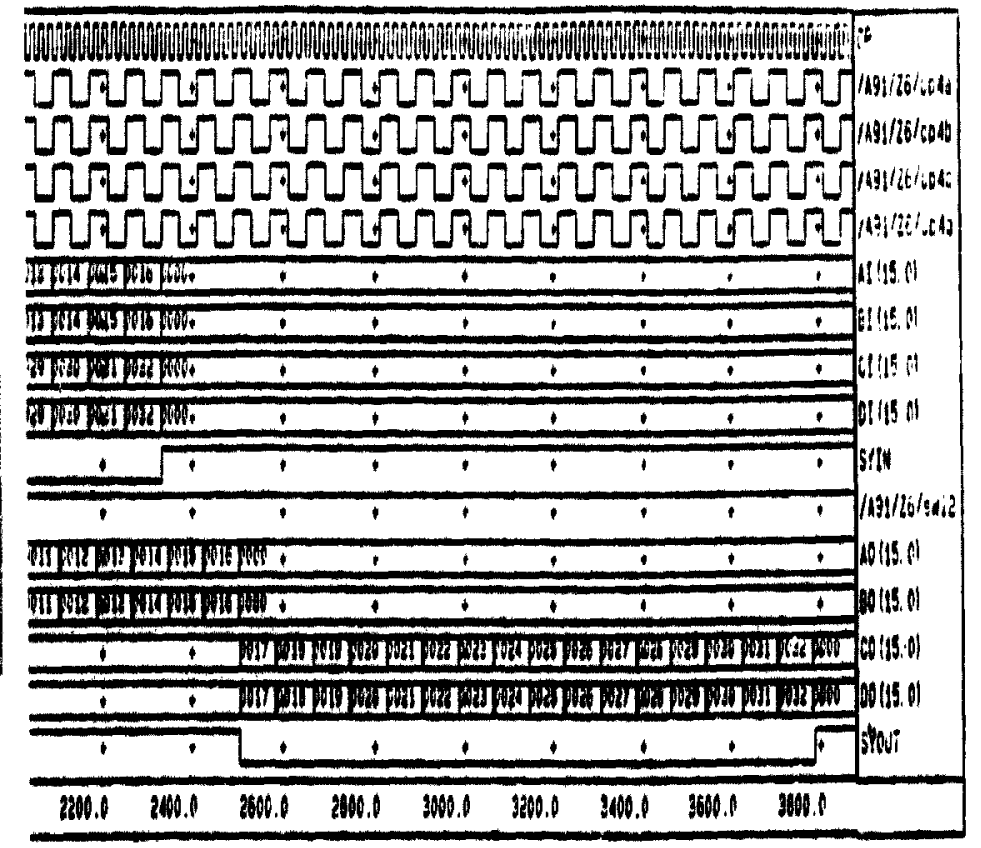

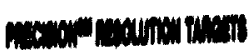

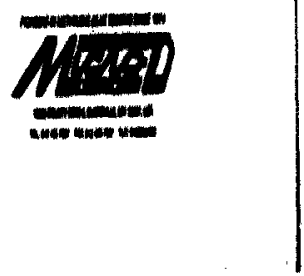

Siculation result of Right svitch mode in Exanple 4 


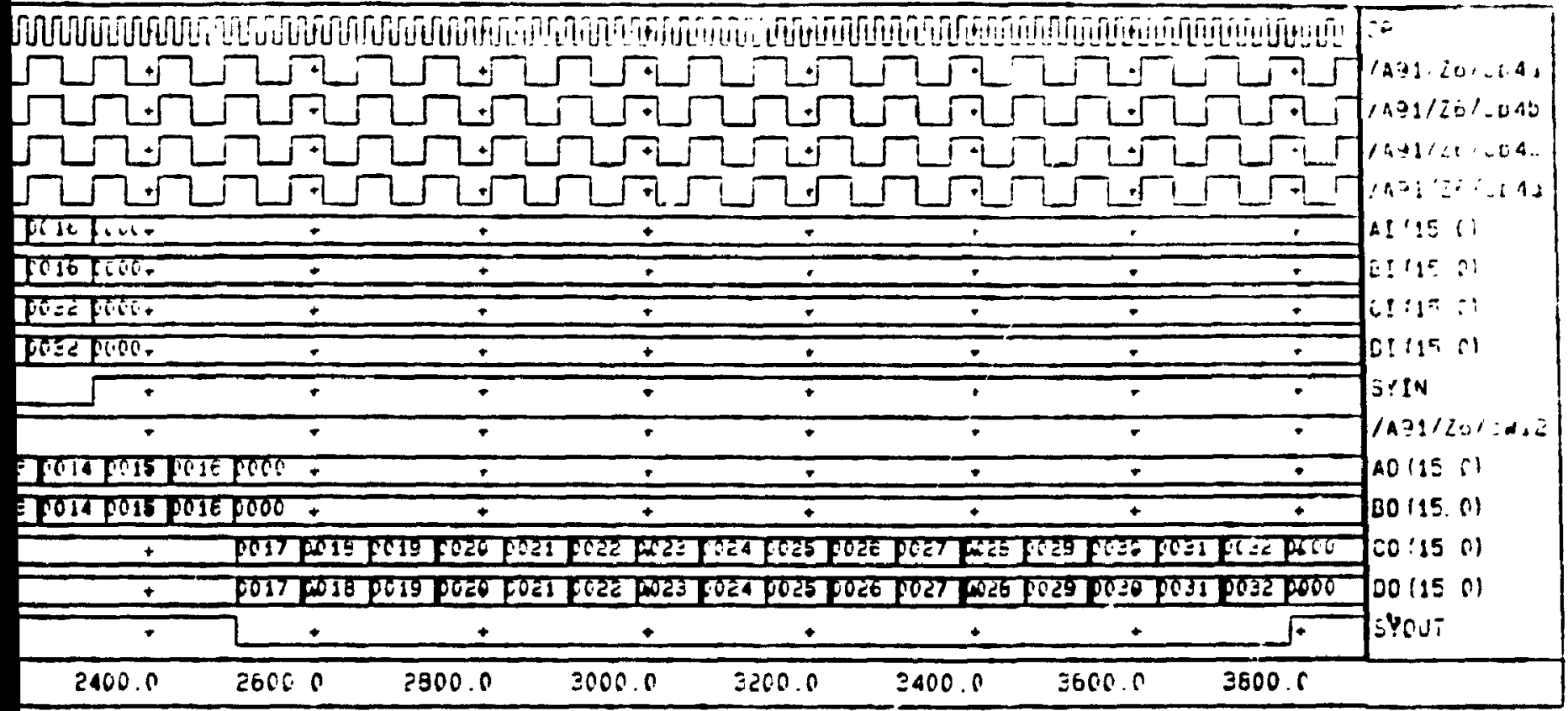

ation result of Right switch mode in Example 4 


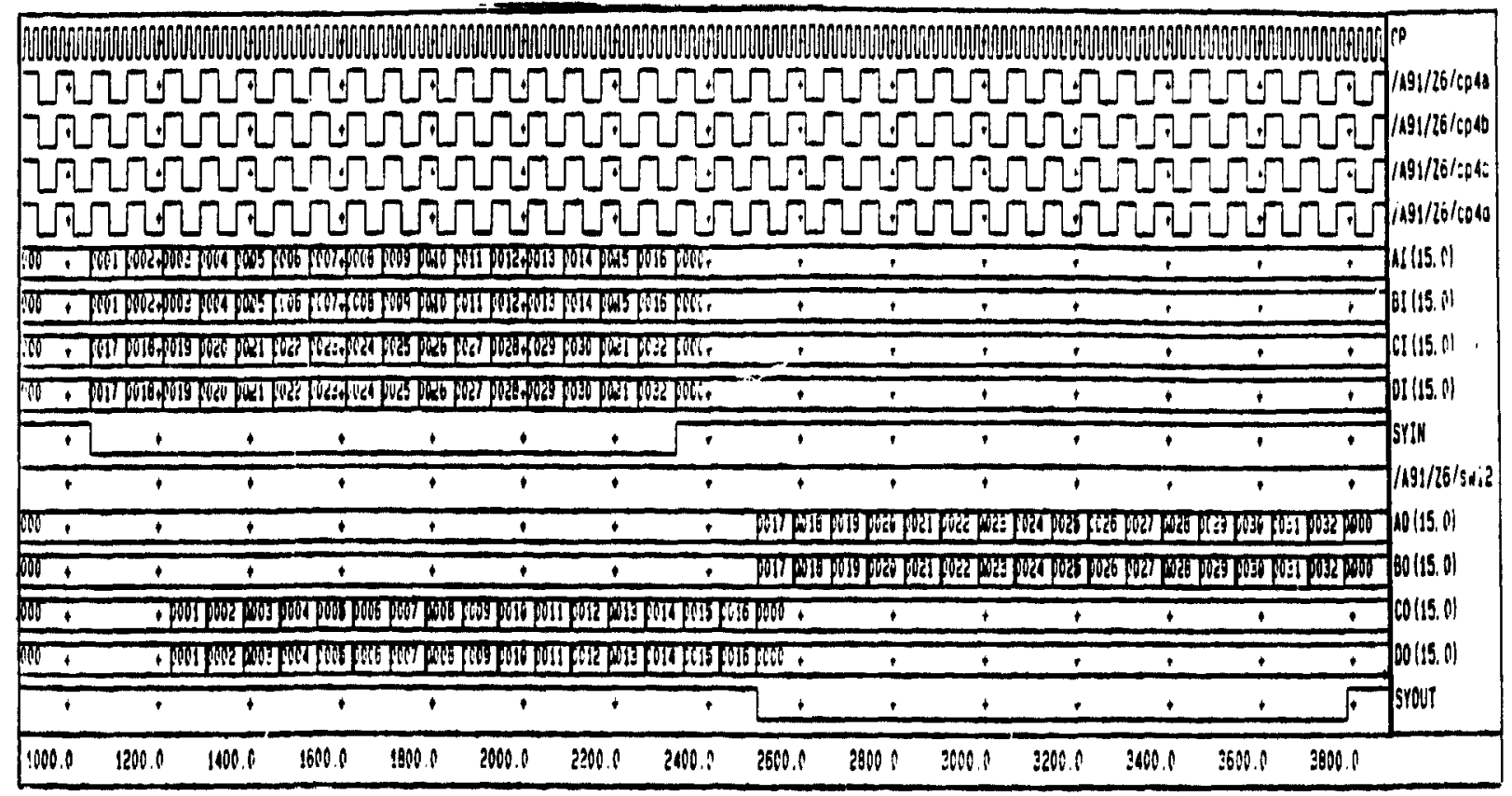

Figure 5.11 Simuiation resulk of Output switch mode in Example 4 


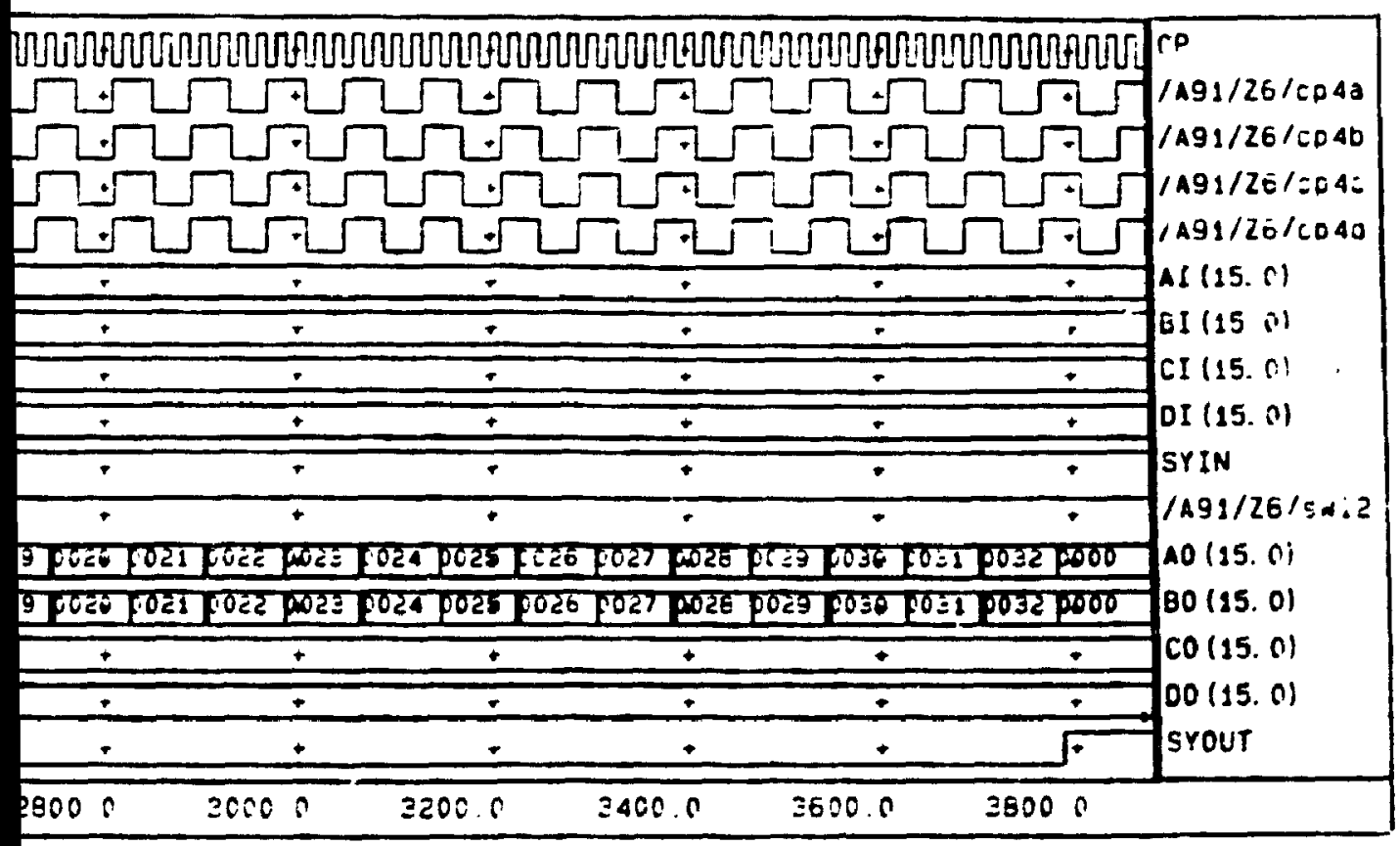

put switch mode in Exampie 4 


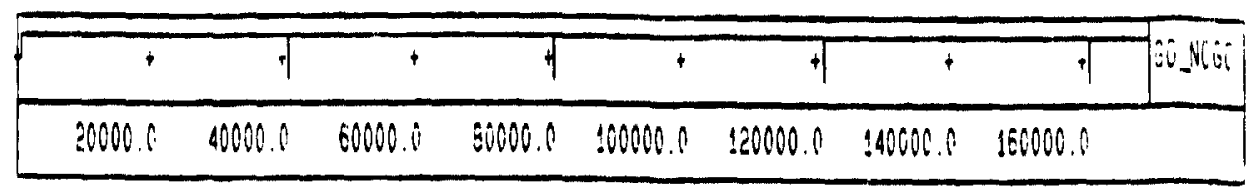

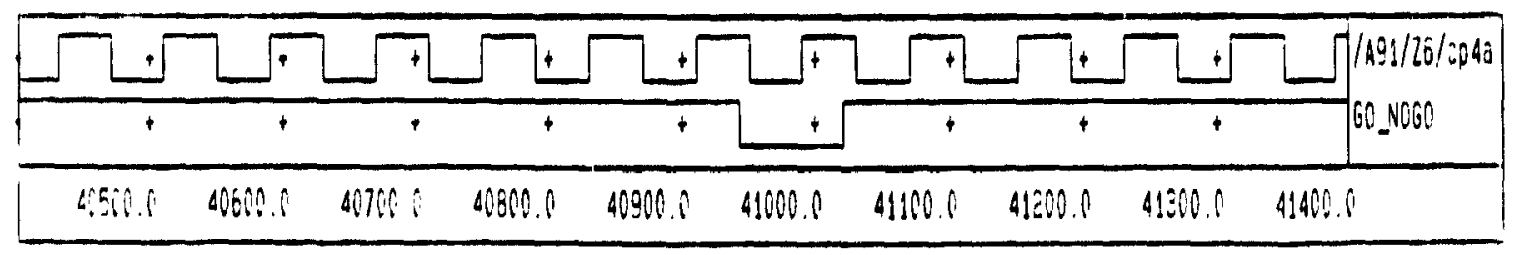

(t)

Figure 5.12 simulated resodnse of the 60.0060 gutput.

(a) For two comoletes crcies (b) A magniffies be NeGo ouise 


\begin{tabular}{|c|c|c|c|}
\hline+1 & $\dot{T}$ & $T$ & 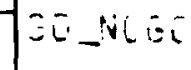 \\
\hline 000.0 & 140000.8 & 150000.0 & \\
\hline
\end{tabular}

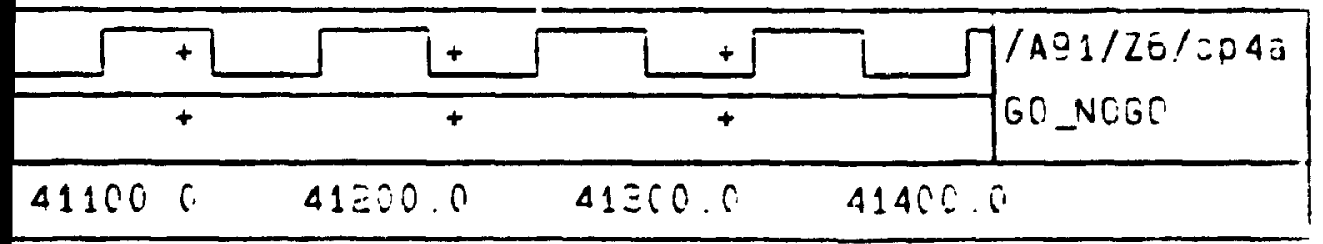

tout.

magnifiea GC NCGO ouise 
are necessary in order to test the tri-state buffers. and to measure the circuit for threshold voltages and current from the power supply. The test pattern for these three simulations are organized depending on the number of the input buffers and their nature. A more detailed explanation of these simulations can be found in Appendix $\mathbf{C}$.

\subsubsection{Cascaded Structure Simulations}

After all switch modes are simulated as stand-alone modules, they are cascaded with either a Switch or a Butterfly for further simulations. The main purpose of these simulations is to verify that the Switch design is capable of operating as part of a cascaded structure. Three different combinations of cascaded structures can be included in an FFT Switch, namely "Switch-Switch". "Switch-Butterfly" and "ButterflySwitch". These are shown in Figure 5.13.

The "Switch-Switch" combination corresponds to two cascaded Switches. Table 5.1 shows all the possibilities of the "Switch-Switch" combination for various switch modes. The input stimulus of the cascaded structure is loaded onto the first Switch and the result of the complete simulation is recorded at the output of the second Switch. Although Table 5.1 shows all the possibilities of the "Switch-Switch" combination, the majority of them are not applicable to the pipelined FFT processor.

Example 6: Figure 5.14 shows the simulation of two cascaded switches where both operate in the Left switch mode. As explained in the previous chapters, this is used primarily to provide the necessary delay before the reordering process. As the simulation shows, since each Switch imposes a sixteen clock cycles delay, data on channel $A$ is ihirty-two clock cycles in advance of data on channel B. No delay is 

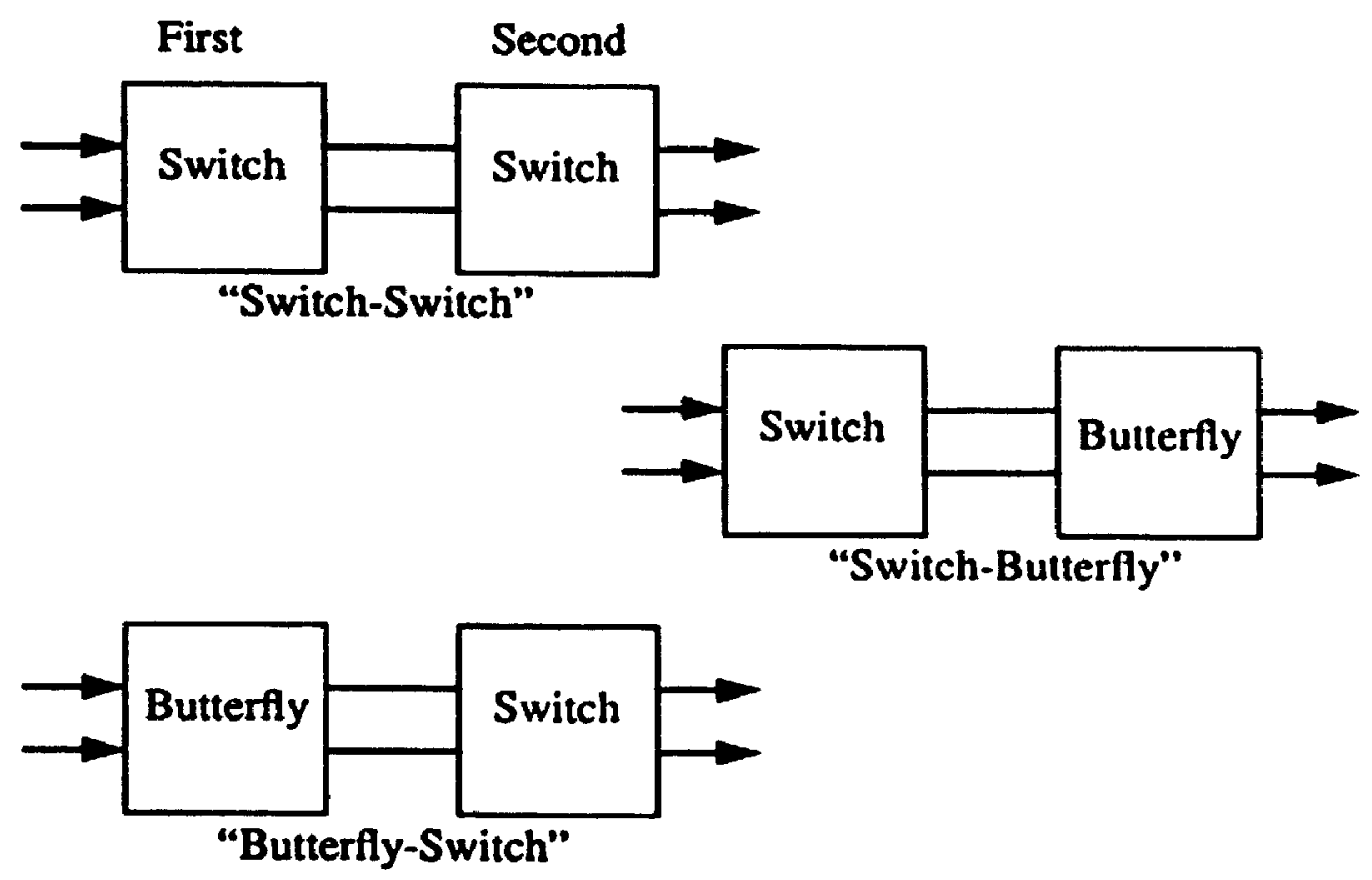

Figure 5.13 Three two modules combinations include a Switch

channel $\mathrm{A}$ is thirty-two clock cycles in advance of data on channel B. No delay is imposed on the Sync pulse, hence it is still three clock cycles in advance of the valid data on channel $\mathbf{A}$.

Example 7: Figure 5.15 shows another "Switch-Switch" simulation result where one Switch operates in End switch mode and the other in Output mode. This is the same combination as in Example 2 of the section 5.2.1.2. When the two simulations are compared, both yield the same output result. The Figure shows that the data stream in both channels and the Sync pulse at the output of the second module are aligned. This is important since the Output mode must represent the last Switch when a number of Switches are cascaded. In order to pass data onto the next Butterfly in the pipeline, the 


\begin{tabular}{|l|ccccc|}
\hline Socond & Right & Left & Output & End & Middle \\
\hline Right & $\sqrt{ }$ & $\mathbf{x}$ & $\mathbf{x}$ & $\sqrt{ }$ & $\mathbf{x}$ \\
Left & $\mathbf{x}$ & $\sqrt{ }$ & $\mathbf{x}$ & $\mathbf{x}$ & $\mathbf{x}$ \\
Output & $\sqrt{ }$ & $\mathbf{x}$ & $\mathbf{x}$ & $\sqrt{ }$ & $\mathbf{x}$ \\
End & $\mathbf{x}$ & $\sqrt{ }$ & $\mathbf{x}$ & $\mathbf{x}$ & $\mathbf{x}$ \\
Middle & $\mathrm{x}$ & $\mathbf{x}$ & $\mathbf{x}$ & $\mathbf{x}$ & $\mathbf{x}$ \\
& & & & & \\
\hline
\end{tabular}

$\sqrt{ }$ Applicable $\quad \mathrm{x}$ Not Applicable

Table 5.1 A table of all possibilities for "Switch-Switch" combination

data stream and Sync pulse must be synchronized and routed correctly at the output of the cascaded structure.

For the "Switch-Butterfly" and "Butterfly-Switch" combinations, shown in Figure 5.13, the FFT Switch is cascaded either in front of or after the Complex Butterfly. Table 5.2 shows all the possibilities allowed for these two combinations. There are five different combinations that are applicable for meaningful simulations out of the ten possible combinations.. 


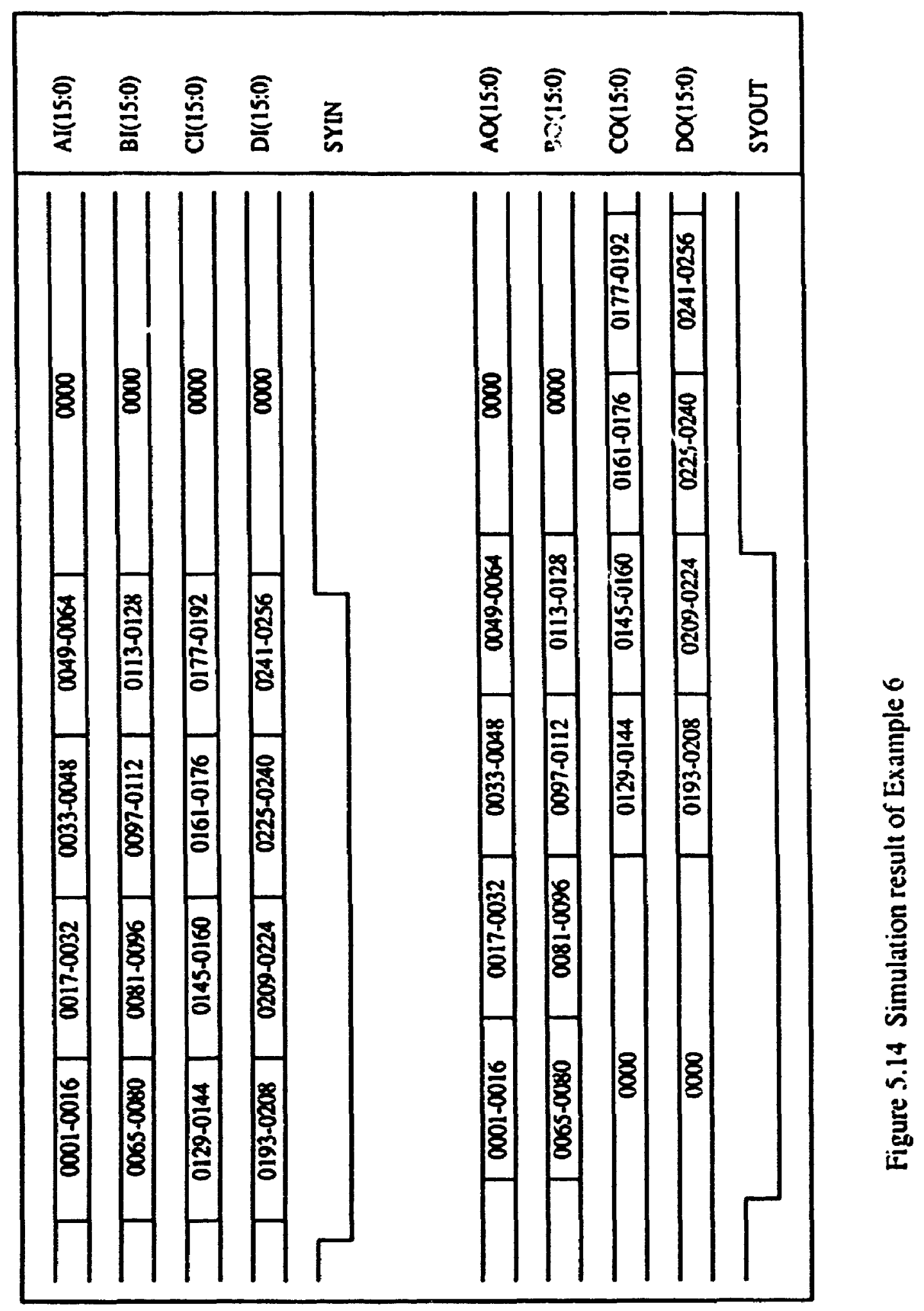




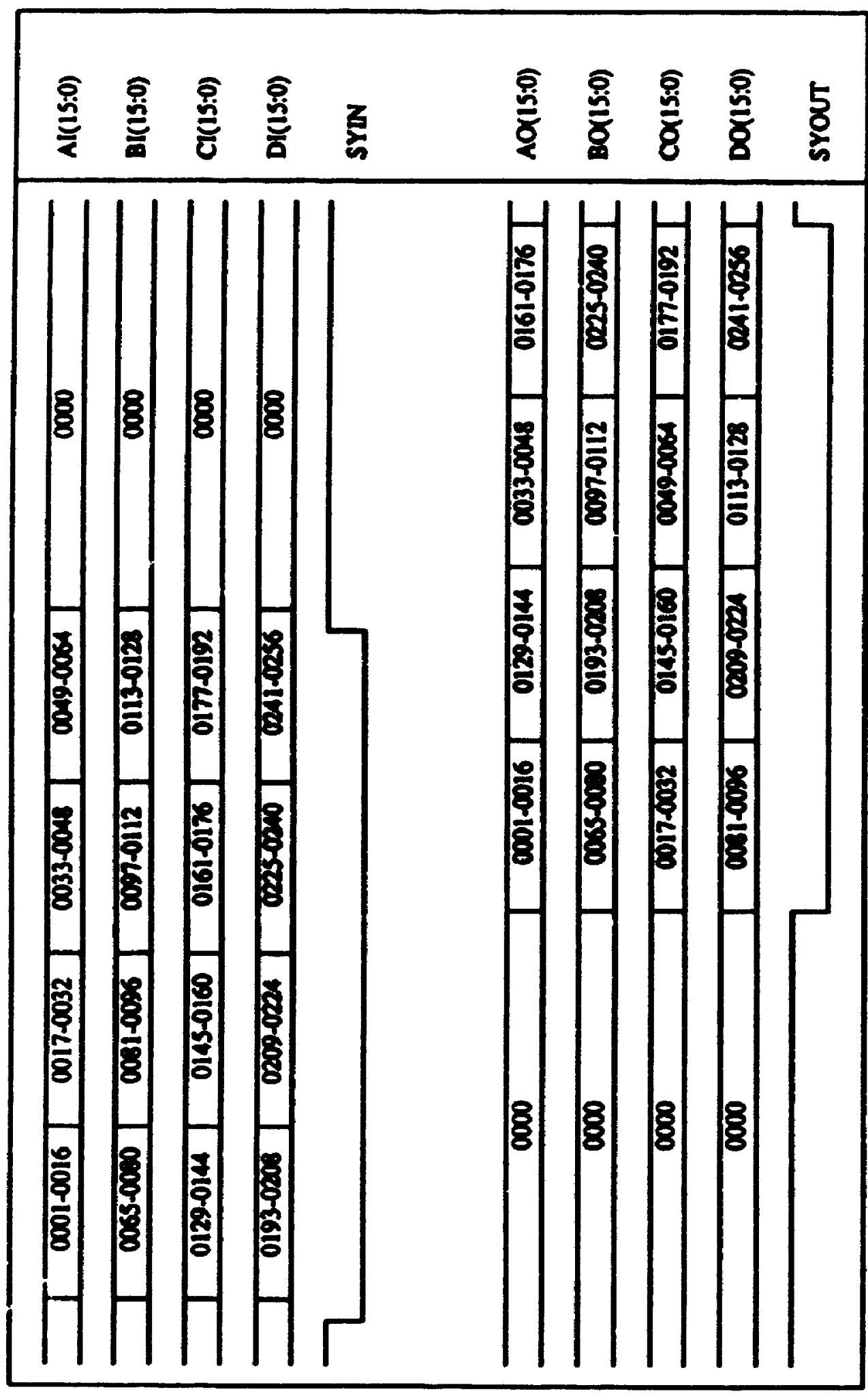

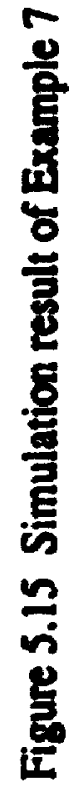




\begin{tabular}{|c|c|c|c|}
\hline \multirow[t]{2}{*}{1} & \multicolumn{2}{|c|}{ Butterfiy } & \multirow[b]{2}{*}{ Applicable } \\
\hline & First & Second & \\
\hline \multirow{5}{*}{ 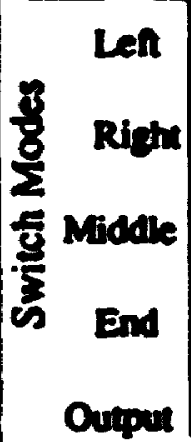 } & $\sqrt{ }$ & $x$ & \\
\hline & $x$ & $x$ & \\
\hline & $\sqrt{ }$ & $\sqrt{ }$ & \\
\hline & $\sqrt{ }$ & $x$ & \\
\hline & $x$ & $\sqrt{ }$ & \\
\hline
\end{tabular}

Table 5.2 "Switch-Butterfly" and "Butterfly-Switch" combinations

\subsection{Prototype for the FFT Switch}

The FFT Switch proworypes were implemented by using $1.5 \mu \mathrm{m}$ HCMOS gate array vechnology from LSI Logic. The wotal gate count of the Switch is 9262 gates distributed over 6134 nets. The $9.98 \mathrm{~mm}^{2}$ die is housed in a 180-pin ceramic package. Post-layout simulations were completed with the collaboration of the Alberta Microelectronics Centre. A die photo of the FFT Switch is shown in Figure 5.16 and the pin layout and bonding diagram are located in Appendices D and E respectively.

\subsection{Prototype Tests}

Ten prototype FFT Switch ASICs were fabricated and functionally tested at the foundry. All ten chips passed the functional lest carried out at 10MHz. These prototypes were further tested at CRC in the self-test mode. A test board was constructed so that it 


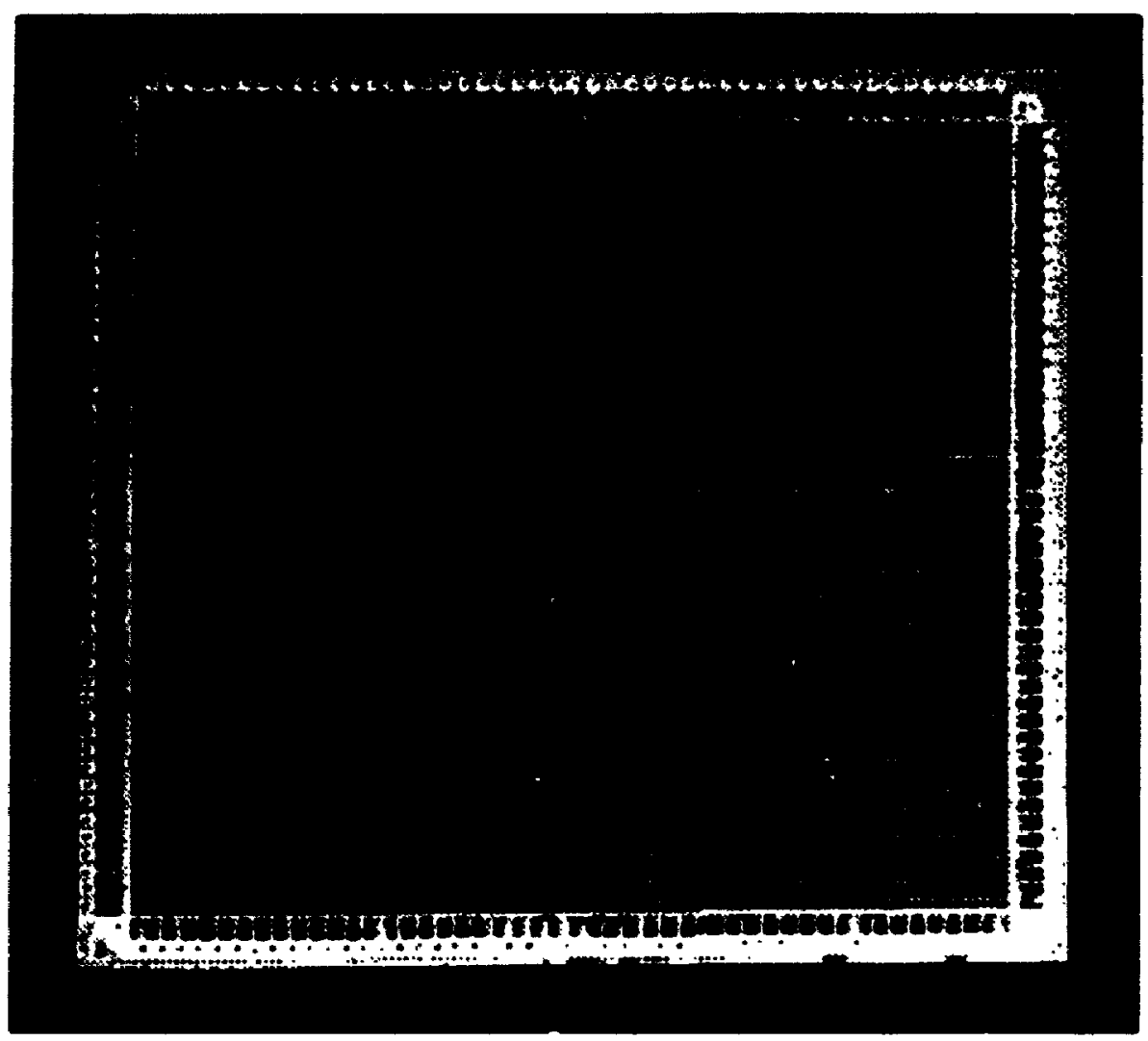

Figure 5.16 A die photo of the FFT Switch

was possible to examine just the self-test mode for both the FFT Switch and Complex Butterfly. A signal generator was equipped to produce the master clock frequencies and a frequency counter was used to observe the signal at the GO_NOGO output. All ten prototypes operated satisfactorily at the master clock frequency of $50 \mathrm{MHz}$ which met the system requirement. The frequency of the signal generator was then increased until the logic low pulse disappeared from the GO_NOGO output in order to find out the maximum operating frequency. The frequency just before the pulse vanished from the 
output was recorded along with the power consumption of the Switch at that frequency.Table 5.3 shows the test results of the ten prototypes which were operated with a power supply of $5 \mathrm{~V}$. Further testing was completed at $3.3 \mathrm{~V}$ to determine the response of the prototype at a lower voltage. The primary reason to reduce the voltage supply was to decrease the power consumption of the Switch and the FFT Processor on the whole. Table 5.4 shov:s the test results of the Switch prototypes at 3.3V.

\begin{tabular}{|c|c|c|}
\hline Prototypes & $\begin{array}{c}\text { Naximum Frequency } \\
\text { Master Clock (MHz) }\end{array}$ & $\begin{array}{c}\text { Current } \\
(\mathrm{mA})\end{array}$ \\
\hline 1 & 150.8 & 350 \\
\hline 2 & 150.2 & 340 \\
\hline 3 & 149.8 & 340 \\
\hline 4 & 151.9 & 350 \\
\hline 5 & 150.4 & 340 \\
\hline 6 & 149.4 & 340 \\
\hline 7 & 150.5 & 340 \\
\hline 8 & 150.2 & 340 \\
\hline 9 & 152.3 & 350 \\
\hline 10 & 150.1 & 340 \\
\hline
\end{tabular}

Table 5.3 Prototype resuits at self-test mode for 5.0V power supply

The Switch prototypes were found to operate in the ranges of 149 to $152 \mathrm{MHz}$ at $5 \mathrm{~V}$ and 97 to $101 \mathrm{MHz}$ at $3.3 \mathrm{~V}$. The power dissipation at $150 \mathrm{MHz}$ and $5 \mathrm{~V}$ was $1.70 \mathrm{~W}$ and at $100 \mathrm{MHz}$ and $3.3 \mathrm{~V}$ was $0.43 \mathrm{~W}$. 


\begin{tabular}{|c|c|c|}
\hline Prototypes & $\begin{array}{c}\text { Maximum Frequency } \\
\text { Master Clock }(\mathrm{MHz})\end{array}$ & $\begin{array}{c}\text { Current } \\
(\mathrm{mA})\end{array}$ \\
\hline 1 & 97.4 & 130 \\
\hline 2 & 100.1 & 130 \\
\hline 3 & 100.6 & 130 \\
\hline 4 & 100.1 & 130 \\
\hline 5 & 100.1 & 130 \\
\hline 6 & 100.6 & 130 \\
\hline 7 & 99.6 & 130 \\
\hline 8 & 100.2 & 130 \\
\hline 9 & 101.0 & 130 \\
\hline 10 & 96.7 & 130 \\
\hline
\end{tabular}

Table 5.4 Prototype results at self-test mode for 3.3V power supply

At the time of writing, a 32 point pipelined FFT processor board is being built. Both the FFT Switch and the Complex Butterfly will be tested together on this processor board.

\subsection{Summary}

The simulations of the FFT Switch were performed under the best, the nominal and the worst case commercial conditions. All functions of the Switch operated satisfactorily under all of these conditions and met the system throughput requirement of $25 \mathrm{Msample/sec} \mathrm{per} \mathrm{channel.} \mathrm{Ten} \mathrm{prototypes} \mathrm{were} \mathrm{fabricated} \mathrm{by} \mathrm{LSI} \mathrm{Logic} \mathrm{and} \mathrm{the}$ self-test procedure was performed on all ten prototypes initially with the system 
requirement of $50 \mathrm{MHz}$ as the frequency of the master clock. Further testing was completed to investigate the maximum operational master clock frequency of each prototype in self-test mode. 


\section{Chapter Six}

\section{Conclusions}

\subsection{Summary}

The increasing demand for low-cost and simple earth stations for satellite communications has signalled the need for more efficient techniques to tackle the issue. One such technique involves the use of frequency-division multiple access (FDMA) as the up-link to satellites and time-division multiple access (TDMA) as the down-link to ground stations. The required transformation of FDMA to TDMA is executed on-board the satellite. The multi-carrier demodulator (MCD) is responsible for the digital signal processing and part of the MCD is a demultiplexer. The demultiplexer can be implemented by using a digital polyphase filter bank in cascade with an FFT processor. A pipelined FFT processor, which can be implemented by using two generic building blocks, namely an FFT Switch and a Complex Butterfly, has been designed such that it is capable of supporting throughput of tens of millions of samples per second. The 
implementation of such an FFT Switch for the radix-2 algorithm is described in this thesis.

The in-place computation and regular structure of the FFT algorithm permits the implementation of either a forward or an inverse pipelined FFT by means of the satme two generic building blocks. By mapping the FFT algorithm onto a one dimensional systolic array, the pipelined processor is able to offer speed improvement over the traditional Von-Neumann architecture. A trade-off between processing speed, flexibility and external control/hardware support is demonstrated by some processor architectures. Though a number of proposed FFT processors can operate at a very high rate of throughput, they lack the flexibility to implement the wide range of transform sizes and the high data throughputs offered by this chip set in a parallel pipelined architecture. Furthermore, the chip set employs a distributed control methodology in order to minimize the system control overheads, thus reducing external circuitry at the system level. Although IC testing is essential for circuit verification, there has been no mention in the literature on pipelined FFT architectures which address the issue of testing by means of built-in self-test technique. The incorporation of BIST enables the FFT Switch to operate off-line testing with minimum external test equipment.

The FFT Switch is fabricated in $1.5 \mu \mathrm{m}$ HCMOS gate array technology. Simulations have shown that the FFT Switch is able to support the data throughput of $25 \mathrm{Msamples} / \mathrm{sec}$ per channel, which represents the minimum system requirement. It is possible to increase the processing power of a single pipeline through the use of a parallel pipelined FFT configuration, which reduces the processing time at the expense of extra hardware. 


\subsection{Future Work}

As the density of integrated circuits grows and the geometry of transistors decreases, the propagation delay due to packaging and interconnects between modules is becoming a significant portion of total signal delay. As mentioned briefly in Chapter 2. the multichip module (MCM) offers a considerable improvement in system level performance due to advances in packaging techniques. By placing several tested dies on a substrate and utilizing the substrate as interconnects between these dies, it is possible to reduce the overall propagation delay of the system.

During the design of the FFT Switch, the possibility of implementing an FFT processor MCM was always a consideration on our part. Attempts have been made to address architecturally a number of issues such as testability, power consumption, number of pin out and so on. Although the idea of MCM is not new, due to technological constraints, it is only recently that a great deal of interests and activity has been directed into this area. Moreover, no reliable and efficient CAD tools have been available on the mariet thus far and the technology has yet to mature.

Through implementation as a multichip module (a 32 point processor consists of four FFT Switches and five Complex Butterflies, a total of over 100,000 gates), the FFT processor may assist in identifying some areas where further investigations and refinements are required. 


\section{References}

[1] J. W. Cooley and J. W. Tukey, "An algorithm for the machine calculation of complex Fourier series," Math. Comp., Vol.19, pp.291-301, April 1965.

[2] W. H. Yim, C. C. D. Kwan, F. P. Coakley and B. G. Evans, "Multi-carrier Demodulators for On-board Processing Satellites," International Journal of Satellite Communications, Vol. 6, pp. 245-251, 1988.

[3] E. Del Re and R. Fantacci, “ Alternative for On-borad Digital Multicarrier Demodlation," International Journa! of Satellite Communications. Vol. 6. pp.267-281, 1988.

[4] E. O. Brigham, "The Fast Fourier Transform," Prentice-Hall, 1974.

[5] A. Oppenheim and R. Schafer, "Discrete-Time Signal Processing," pp. 581622, Prentice-Hall, 1989.

[6] L. Rabiner and B. Gold, "Theory and Application of Digital Signal Processing," pp. 573-614, Prentice-Hall, 1975.

[7] G. D. Bergland, "Fast Fourier Transform Hardware Inplemenations - A Jurvey," IEEE Trans. on Audio and Electroacoutics, Vol. Au-17, No.2, pp.109-119, June 1969. 
[8] R. C. Signleton, "An Algorithm for Computing the Mixed Radix Fast Fourier Transform," IEEE Trans. on Audio and Electroacoustics, Vol. AU17. No.2, pp. 93-103, June 1969.

[9] R. M. Gibson and D. P. McCabe, "Fourier Transform Algorithm Implementations on a General-Purpose Microprocessor," Proc. IEEE ICASSP'81, Vol. 1, pp. 670-672, 1981.

[10] C. R. Jesshope, "The Implementatioon of Fast Radix 2 Tiansforms on Array Processors," IEEE Trans. on Computer, Vol. C-29, No. 1, pp.20-27, January 1980.

[11] P. Papamichalis, "FFT Implementation on the TMS320C30," Proc. 1988 Int'l. Conf. on Acoustics, Speech and Signal Processing, pp.1399-1402, April 1988.

[12] S. Sweitzer, "A Low Cost FFT Chip Set," Proc. IEEE ICASSP'84, Vol. 3 , pp. 44.3.1-3, 1984.

[13] E. H. Wold and A. M. Despain, "Pipeline and Parallel FFT Processors for VLSI Implementations," IEEE Computers, Vol. C33, No.5, pp.414-426, May 1984.

[14] K. O. Siomalas and B. A. Bowen, "Synthesis of Efficient Pipelined Architectures for Implementing DSP Operations," IEEE Trans. on Acoustics, Speech and Signal Processing, Vol. ASSP-33, No.6, pp.1499-1508, December 1985. 
[15] A. Oppenheim and R. Schafer, “Digital Signal Processing," Prentice-Hall.

[16] J. A. Johnston, "Parallel Pipeline Fast Fourier Transformer," IEEE proceedings, Vol 130 part F, No.6, October 1983.

[17] J. A. Johnston, “ Input/output memory for digital convolution via the parallel pipeline fast Fourier transformer," Signal Processing, Elsevier Science Publishers, pp.193-199, 1986.

[18] J. L. Van Meerbergen and F. J. Van Wyk, "A 256-Point Discrete Fourier Transform Processor Fabricated in a $2 \mu \mathrm{m}$ NMOS Technology," IEEE Journal of Solid-State Circuits, Vol. SC-18, No.5, pp. 604-609, October 1983

[19] G. D. Covert, "A 32 Point Monolithic FIT Processor Chip," Proc. IEEE ICASSP'82, pp. 1081-1083, 1982

[20] R. W. Linderman, C. G. Shephard, K. Taylor, P. W. Coutee, P. C. Rossbach and J. M. Collins, “A 70MHz 1.2 Micron CMOS 16-Point DFT Processor," IEEE Custom Integrated Circuits Conference, pp. 219-222, i 987.

[21] C. P. S. Yeung, C. H. Chan, T. A. Kwasniewski, V. Szwarc and L. Desormeaux, "A Cascadeable Pipeline Fast Fourier Trnasform Switch With Built-in Self-Test," Proceedings of CCVLSI '91 (Canadian Conference on Very Large Scale Integrated circuit), pp. 3.5.1-3.5.8, August 1991.

[22] W. Wong, C.H. Chan, T.A. Kwasniewski, V. Szwarc and L. Desormeaux, "A modular 25 MSamples/sec Complex-Butterfly implementation using 
redundant binary arithmetic and built-in self test techniques," Proceedings of CCVLSI '90 Conference, pp. 9.5.1-9.5.8, 1990.

[23] E. E. Swartzlander, Jr. and G. Hallnor, "Fast Transform Processor Implementation," Proc. IEEE ICASSP'84, pp. 25A.5.1-4, 1984.

[24] J. Fox, G. Surace and P. Thomas, "A Self-Testing $2-\mu \mathrm{m}$ CMOS chip set for FFT applications," IEEE Journal of Solid-State Circuits, Vol. SC-22, No.1, pp. 15-19, February 1987

[25] E. E. Swartzlander, Jr., V. K. Jain and H. Hikawa, "A Radix-8 Wafer Scale FFT Processor," in VLSI Signal Processing IV, Ed. H. S. Moscovitz, K. Yao and R. Jain, New York, IEEE Press, 1991.

[26] K. Yamashita, A. Kanasugi, S. Hijiya, G. Goto, N. Matsumura and T. Shirato, "A Wafer-Scale 170000-Gate FFT Processor with Built-In Test Circuits," IEEE Journal of Solid-State Circuit, Vol. 23, No. 2, pp.336-342, April 1988.

[27] P. Bardell, W. McAnney and J. Savir, "Built-In Test for VLSl: Pseudorandom Techniques," New York: John Wiley \& Sons, 1987.

[28] E. J. McCluskey, "Logic Design Principles: with emphasis on testable semicustom circuits," Prentice-Hall International Edition, 1986.

[29] S.W. Golumb, "Shift Register Sequences," Aegean Park Press, Laguna Hills, Calif., 1982 
[30] S.Y.Hassan and E.J. McCluskey, "Increased Fault Coverage Through Multiple Signature," Digest of IEEE Conference on Fault-Tolerant Computing, pp.354-359, 1984.

[31] C.J. Bartlett, J.M. Sgelken and N.A. Teneketges, "Multichip Packaging Design for VLSI-Based Systems," IEEE Trans. Comp., Hybrids, Manuf. Technology, Vol. CHMT-12, no. 4, pp. 272-284, December 1987.

[32] L. Desormeaux, V. Szwarc, J. Rostworowski, C. Lapadat, C. Harris, "The Design of a Cascadable FIR Filter Multichip Module," Proceedings of the Canadian Conference on Electrical and Computer Engineering. pp. 13.3.1-13.3.4, Septemter, 1991 .

[33] Implementing Fast Fourier Transform Systems with the L64280/81 Chip Set, DSP application note number one, LSI Logic, Milpitas, CA., April 1990.

[34] LH9124 Digital Signal Processor and LH9320 Address Generator by Sharp Electronics. 


\section{Appendix A :}

\section{Simulations for Switch B and Switch C in Example 1}



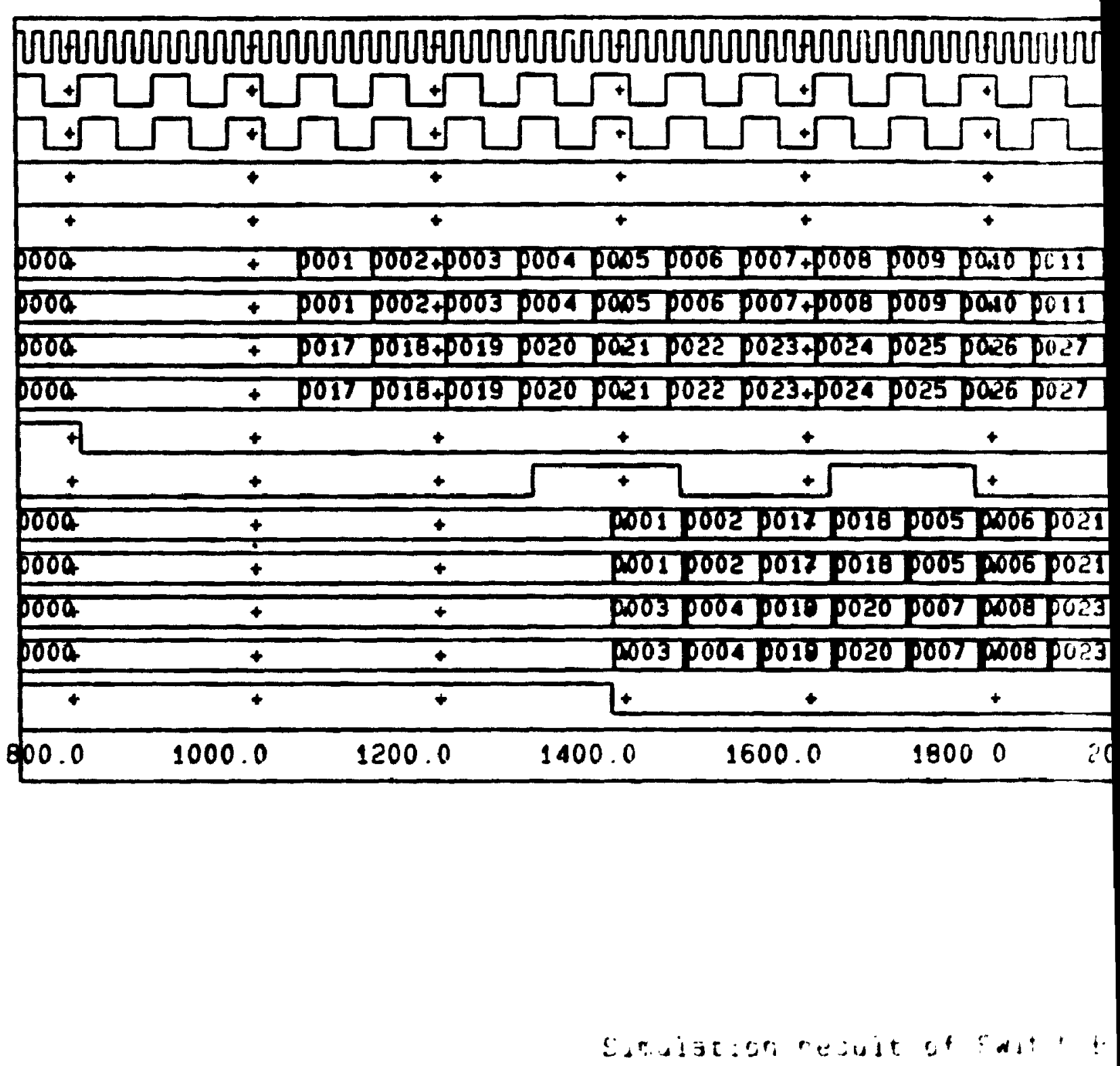


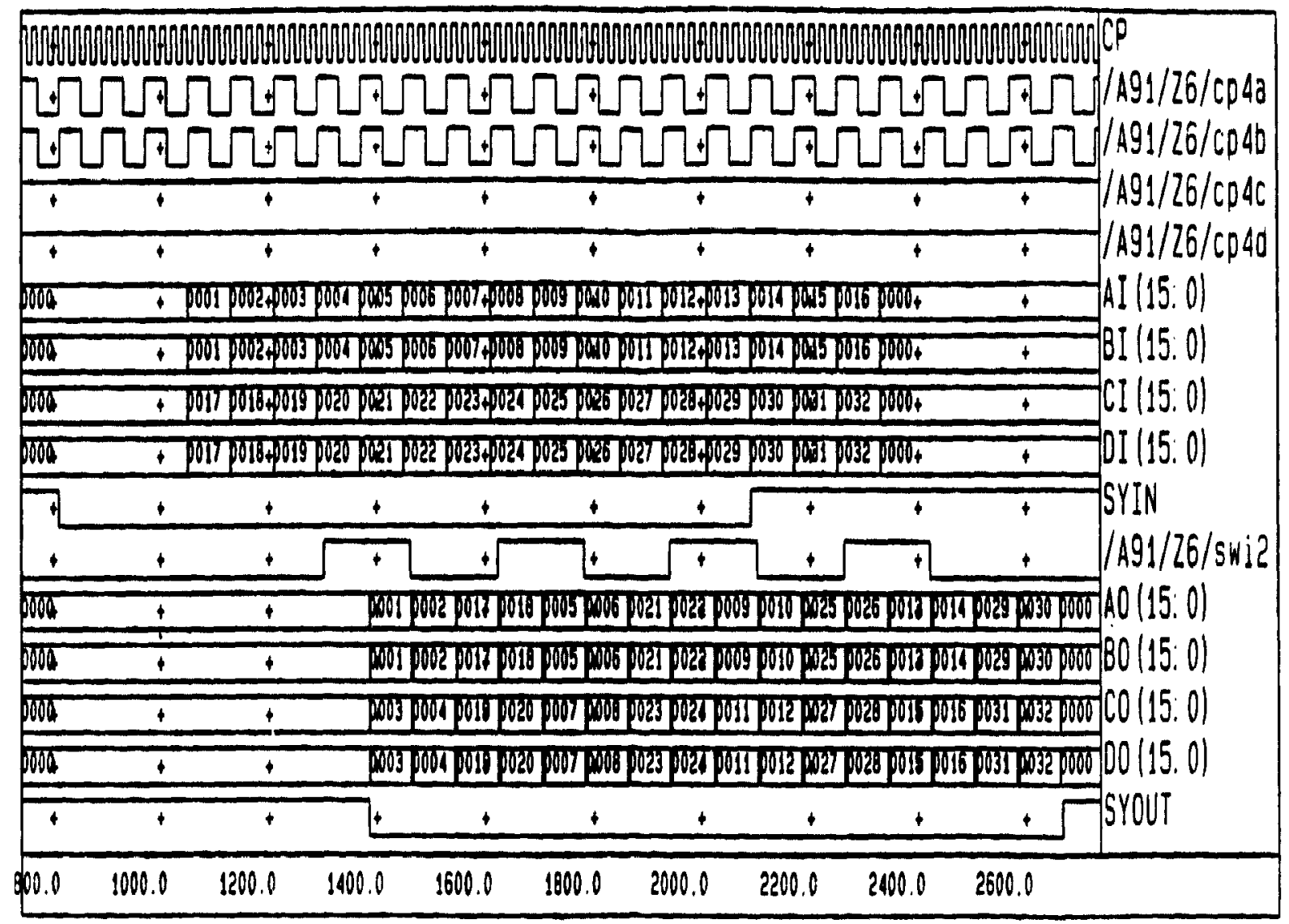




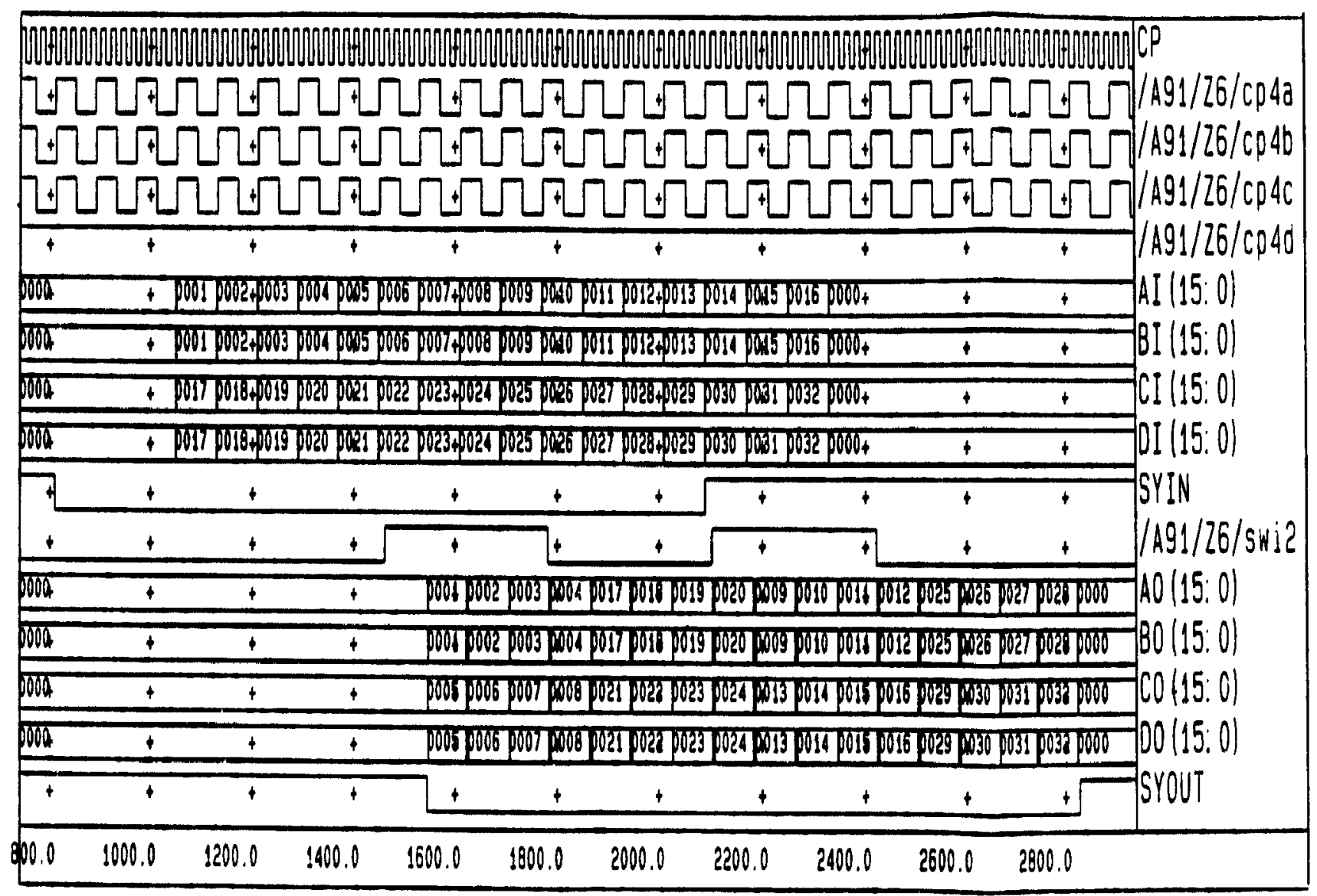

Simulation result of Suitch $C$ in Exampie! 
Appendix B :

Simulations for Left, Right and Output Switch modes in Example 3 


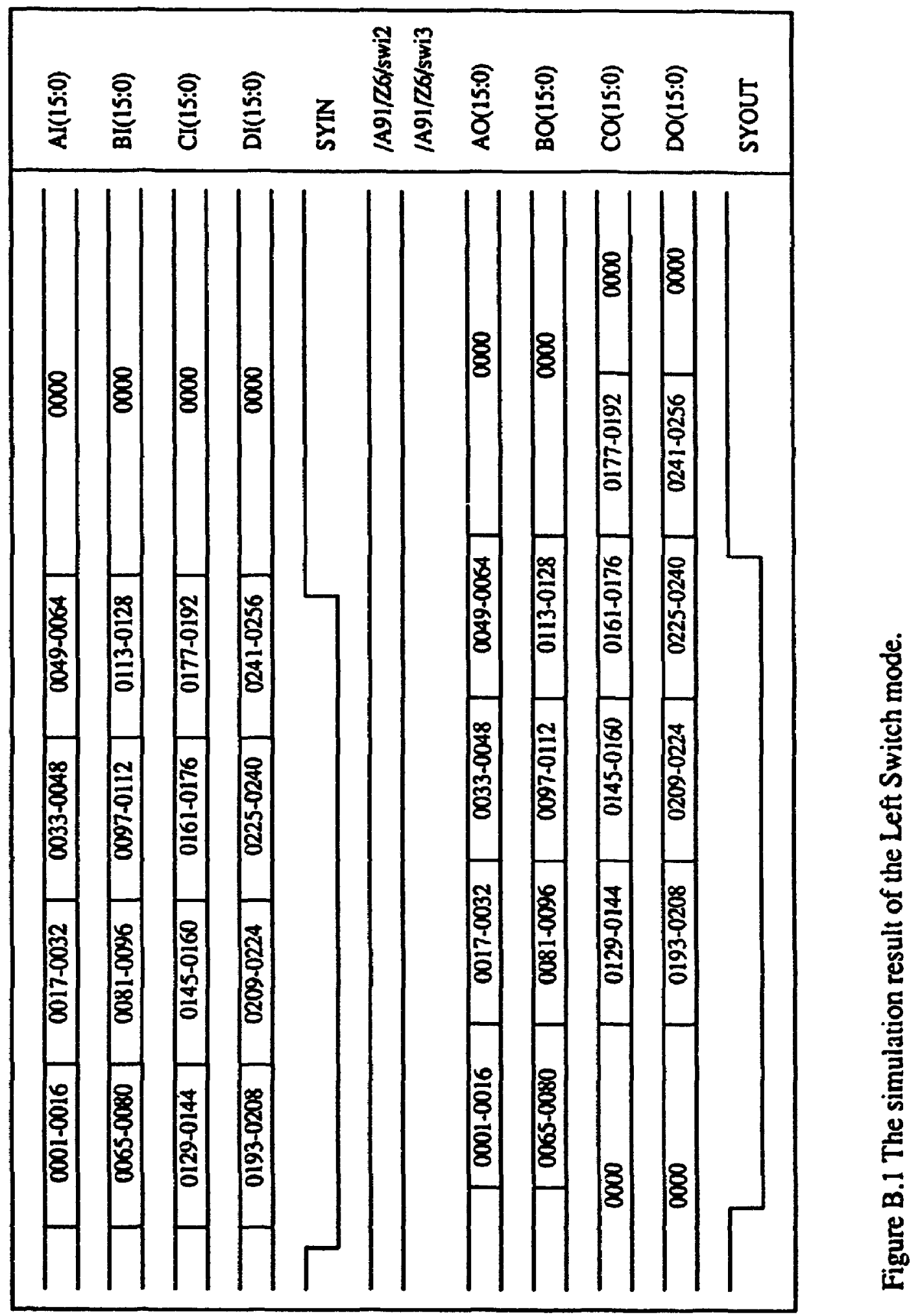




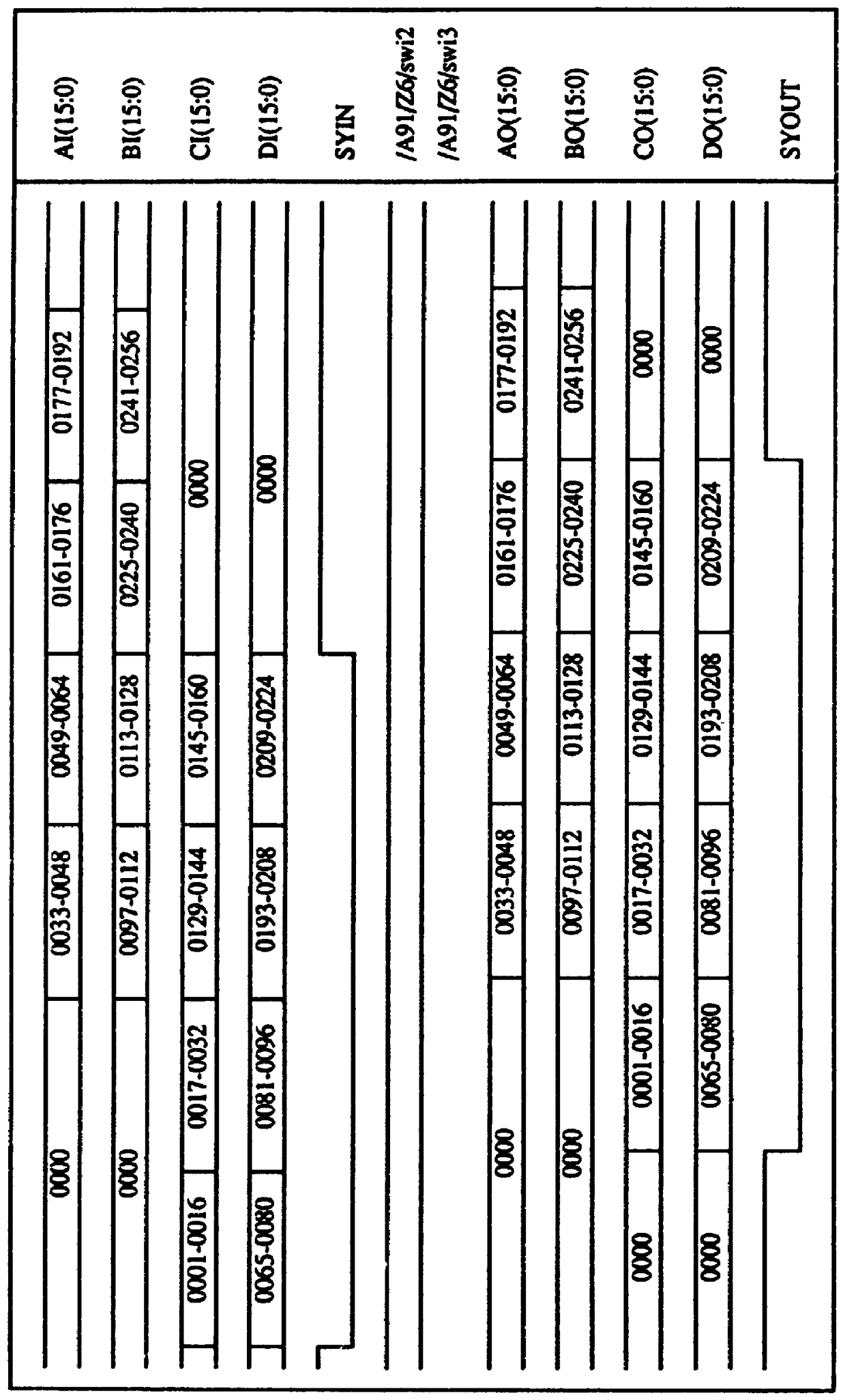

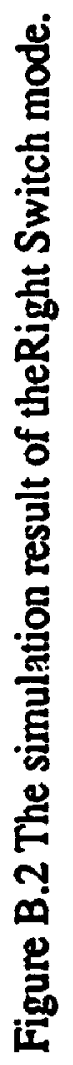




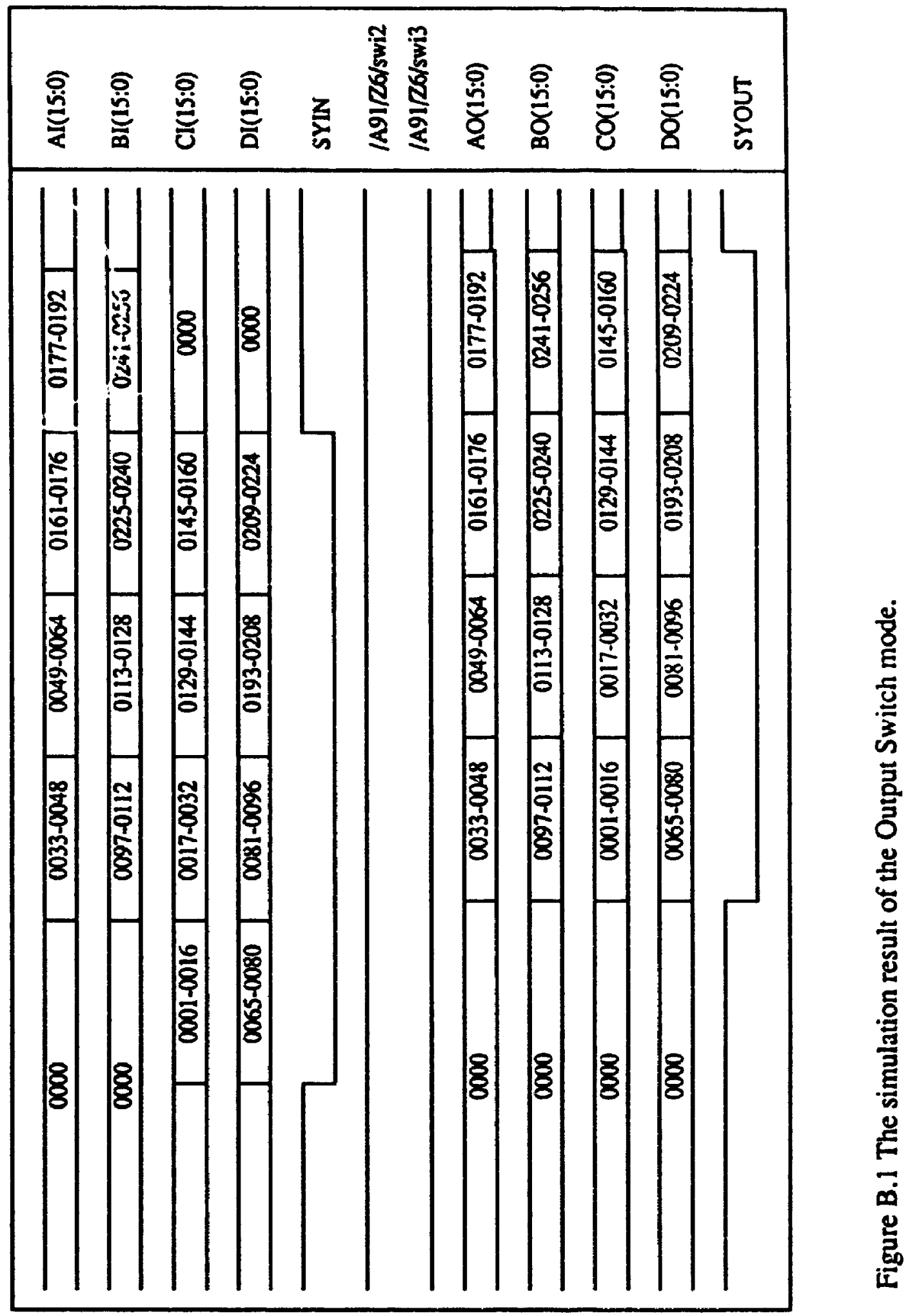




\section{Appendix C :}

\section{Additional notes on the functional simulations necessary for post-fabrication test ${ }^{1}$}

\section{C.1 Introduction}

The functional simulations for post-fabrication is divided into three separate tests:

- to measure the input threshold voltages $\left(V_{I L} / V_{I H}\right)$

- three-states simulation

- to measure power supply current $\left(\mathrm{I}_{\mathrm{dd}}\right)$

A maximum of 12000 test patterns can be used and it is divided into three blocks where each block consists of $\mathbf{4 0 0 0}$ test patterns.

\section{C.2 $\mathbf{V}_{\mathrm{IL}} / \mathrm{V}_{\mathrm{IH}}$ Simulation}

The simulation pattern allows the tester to measure the circuit for input threshold voltages. The simulation is performed under the following conditions: $\mathrm{V}_{\mathrm{dd}}=5 \mathrm{~V}$. Tempature $=25^{\circ} \mathrm{C}$, process $=$ worst case commercial condition and test period $=2000 \mathrm{~ns}$.

To Check input voltage levels accurately, the design must contain $\mathrm{V}_{\mathrm{IU}} / \mathrm{V}_{\mathrm{IH}}$ test circuitry called a "parametric NAND tree". It is possible to build the parametric NAND tree by interconnecting the 2-input NAND gates already attached to the inputs and dedicate one pin as a TESTOUT pin. If a pin cannot be dedicated for use in the

1. From the LSI Logic “Databook and design manua, HCMOS Macrocells and Macrofunclions, Octoher, 1986 
parametric NAND tree, then multiplex the TESTOUT pin with a normal output pin. The tester monitors only the TESTOUT pin when the $V_{\mathbb{I}} / V_{\mathrm{IH}}$ test is executed. Figure C.1 shows a simplified parametric Nand tree. The first $V_{I V} V_{I H}$ test pattern must consist of all ones on the inputs. Initially, the output at TESTOUT is either one or zero depending on the number of inputs (for even number inputs, one is the output at TESTOUT and via versa). As zeros are applied sequentially to the inputs along the parametric NAND tree, the TESTOUT toggles between one and zero.

\section{C.3 Three-state Simulation}

The three-state simulation typically consists of only a few patterns. The patterns must bring all three-state outputs to high impedance at least once during the simulation. When the tester executes the three-state patterns, a test program stipulates that only pins in the high impedance state be monitored. All output pins in the zero state are masked. Execute the three-state simulation under the following conditions: $\mathrm{V}_{\mathrm{dd}}=5 \mathrm{~V}$, Tempature $=25^{\circ} \mathrm{C}$, process=worst case commercial condition and test period=2000ns.

\section{C.4 Idd Simulation}

$I_{d d}$ denotes the power supply current leading from the $V_{d d}$ (power supply) pin into the circuit. The $I_{d d}$ simulation patterns set up the circuit for $I_{d d}$ measurement. The purpose of $I_{d d}$ measurement is to determine how much direct current leakage remains in the circuit after all activity in the circuit has ceased. Normally the $I_{d d}$ simulation very few test patterns and execute under the following conditions: $V_{d d}=5 \mathrm{~V}$, Tempature $=25^{\circ} \mathrm{C}$, process $=$ worst case commercial condition and test period $=2000 \mathrm{~ns}$. 


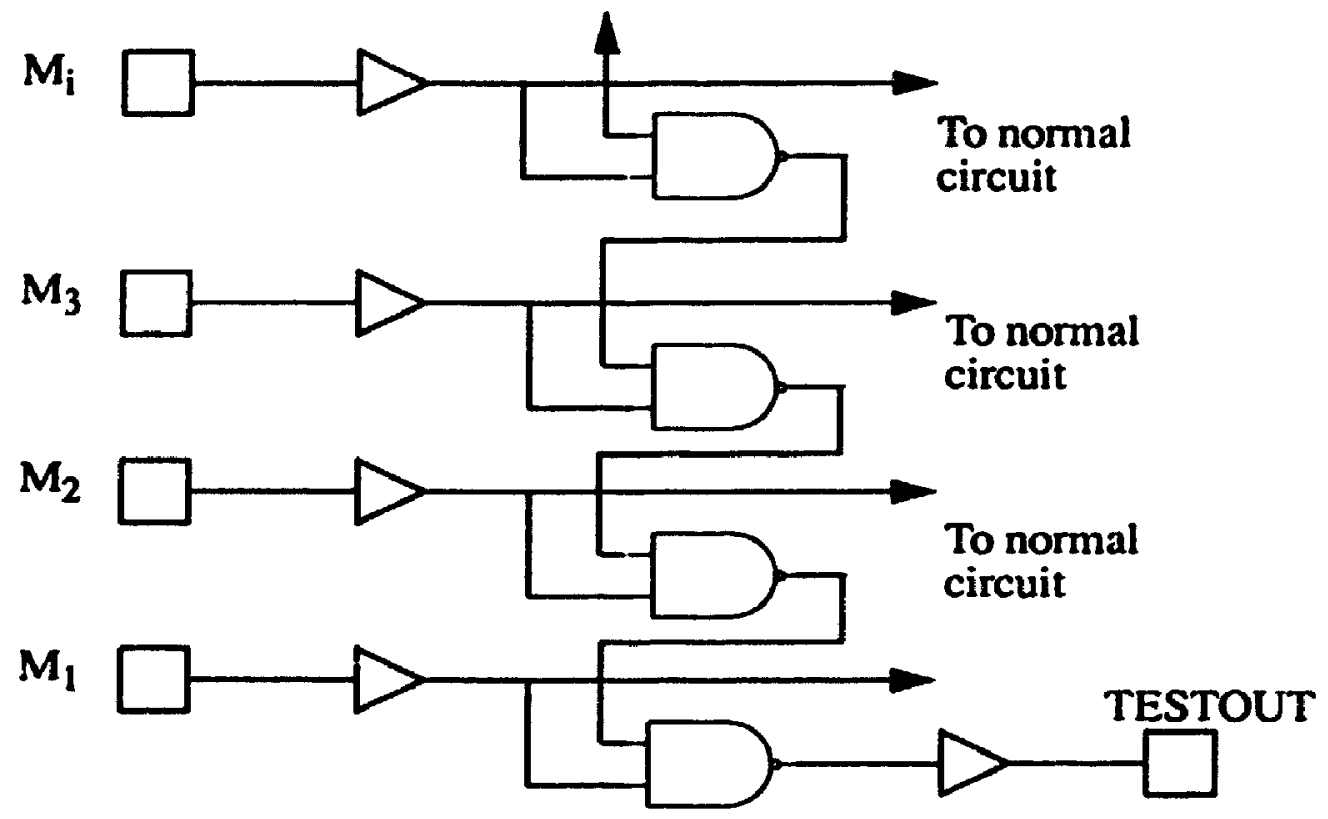

\begin{tabular}{|l|l|l|l|l|l|}
\hline \multicolumn{1}{|c|}{$\mathbf{M}_{1}$} & \multicolumn{1}{c|}{$\mathbf{M}_{2}$} & \multicolumn{1}{c|}{$\mathbf{M}_{3}$} & \multicolumn{1}{c|}{$\mathbf{M}_{\mathrm{i}}$} & $\mathrm{i}=$ even & $\mathrm{i}=$ odd \\
\hline \hline 1 & 1 & $1 \ldots$ & 1 & 1 & 0 \\
\hline 1 & 1 & $1 \ldots$ & 0 & 0 & 1 \\
\hline 1 & 1 & $0 \ldots$ & 0 & 1 & 1 \\
\hline 1 & 0 & $0 \ldots$ & 0 & 0 & 0 \\
\hline 0 & 0 & $0 \ldots$ & 0 & 1 & 1 \\
\hline
\end{tabular}

Figure C.1 Operation of a parametric NAND tree. 


\section{Appendix D :}

\section{The pin layout of the FFT Switch}

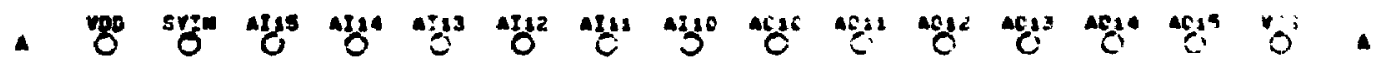

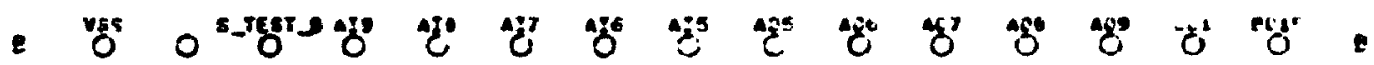

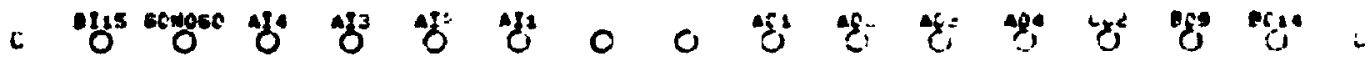

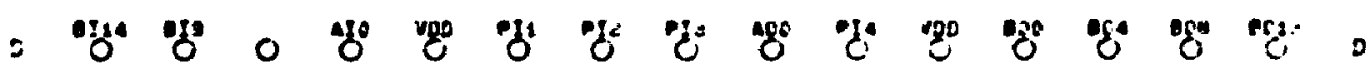

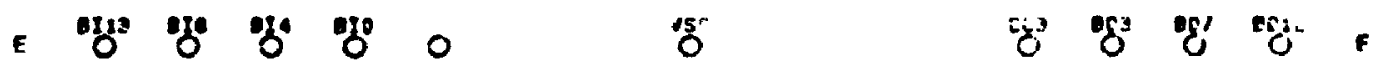

- $y^{2} g^{3} \sin$

- ${ }^{\prime 2}$ og ${ }^{2}$ g

osga og og og:

+ ofg og og no vo

y 8 og 810 .

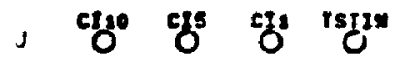

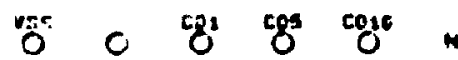

* cyt of cy con

C $\&^{2} \cos \operatorname{cog} 1$

L cgr cj cy

- $8^{3} \quad 8^{7} \operatorname{cog}^{2}$

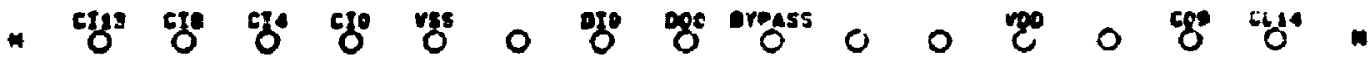

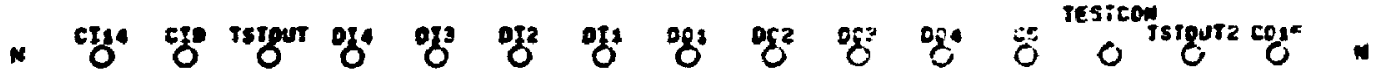

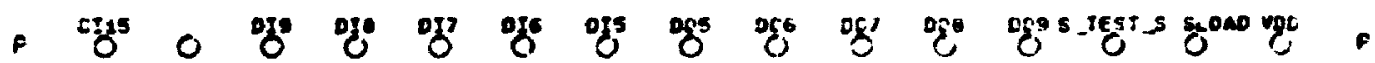

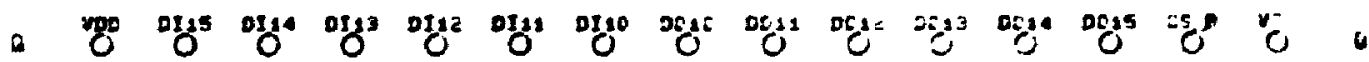

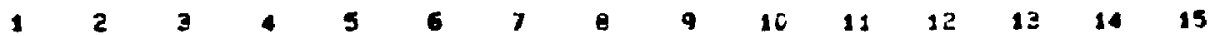

TOP VIEW OF SWITCH PINOUT 


\section{Appendix E :}

\section{Bonding diagram of the FFT Switch}

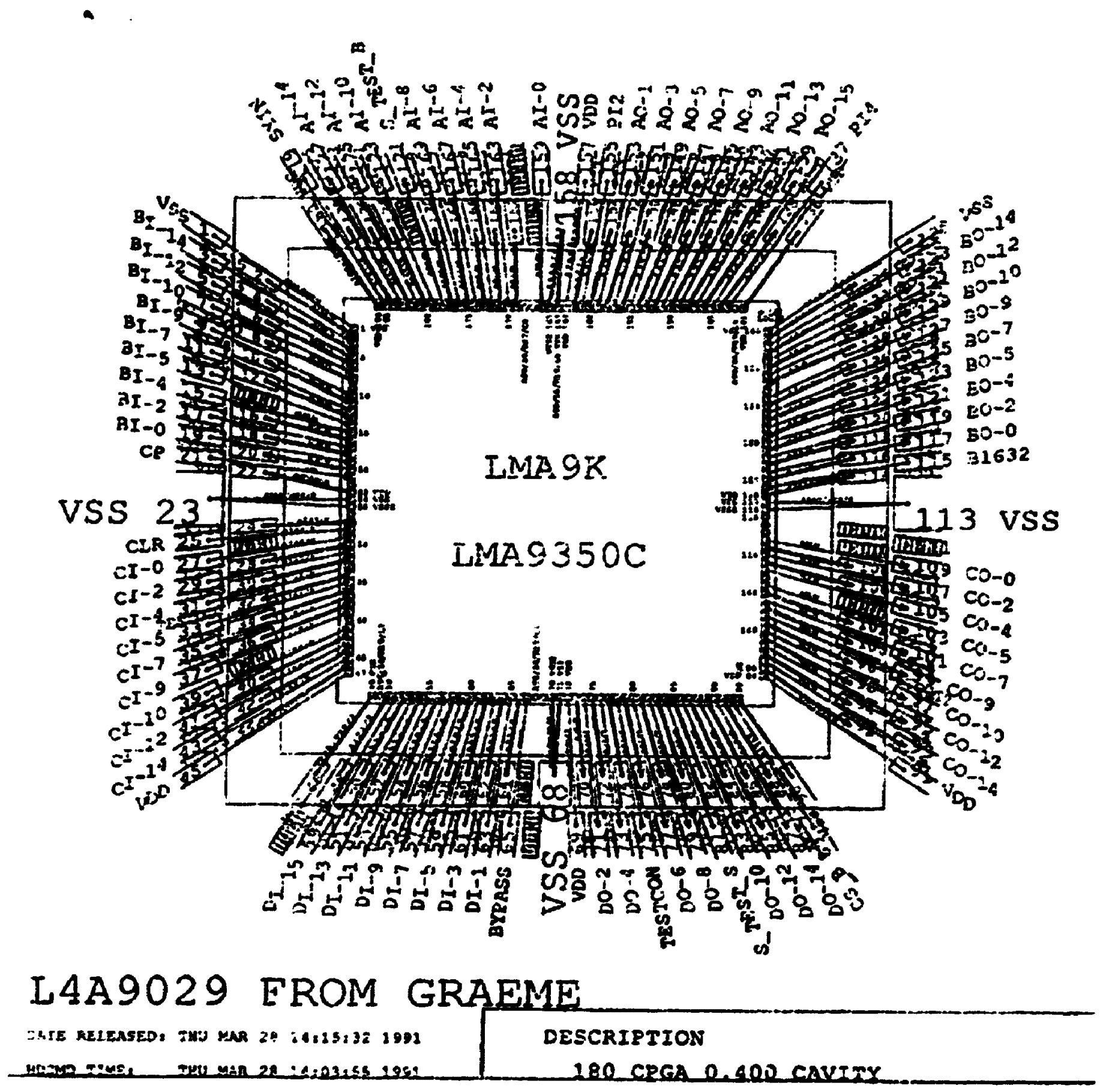

Appendices 
